PREPARED For SUBMission to JCAP

\title{
Dark matter substructure modelling and sensitivity of the Cherenkov Telescope Array to Galactic dark halos
}

\author{
M. Hütten, ${ }^{a, c}$ C. Combet, ${ }^{b}$ G. Maier, ${ }^{a}$ D. Maurin. ${ }^{b}$ \\ ${ }^{a}$ DESY, Platanenallee 6, D-15738 Zeuthen, Germany \\ ${ }^{b}$ LPSC, Université Grenoble-Alpes, CNRS/IN2P3, \\ 53 avenue des Martyrs, 38026 Grenoble, France \\ ${ }^{c}$ Humboldt-Universität zu Berlin, Newtonstraße 15, \\ D-12489 Berlin, Germany \\ E-mail: moritz.huetten@desy.de, celine.combet@lpsc.in2p3.fr, \\ gernot.maier@desy.de,dmaurin@lpsc.in2p3.fr
}

\begin{abstract}
Hierarchical structure formation leads to a clumpy distribution of dark matter in the Milky Way. These clumps are possible targets to search for dark matter annihilation with present and future $\gamma$-ray instruments. Many uncertainties exist on the clump distribution, leading to disputed conclusions about the expected number of detectable clumps and the ensuing limits that can be obtained from non-detection. In this paper, we use the CLUMPY code to simulate thousands of skymaps for several clump distributions. This allows us to statistically assess the typical properties (mass, distance, angular size, luminosity) of the detectable clumps. Varying parameters of the clump distributions allows us to identify the key quantities to which the number of detectable clumps is the most sensitive. Focusing our analysis on two extreme clump configurations, yet consistent with results from numerical simulations, we revisit and compare various calculations made for the Fermi-LAT instrument, in terms of number of dark clumps expected and the angular power spectrum for the Galactic signal. We then focus on the prospects of detecting dark clumps with the future CTA instrument, for which we make a detailed sensitivity analysis using open-source CTA software. Based on a realistic scenario for the foreseen CTA extragalactic survey, and accounting for a post-trial sensitivity in the survey, we show that we obtain competitive and complementary limits to those based on long observation of a single bright dwarf spheroidal galaxy.
\end{abstract}

Keywords: dark matter simulations, semi-analytic modeling, gamma ray experiments 


\section{Contents}

1 Introduction $\quad 1$

2 Modelling the $\gamma$-ray-emission from Galactic DM subhalos 3

2.1 Modelling approach $\quad 4$

2.2 Milky Way dark matter halo 5

2.3 Substructure properties and set of models 5

3 Global properties of the models $\quad 9$

3.1 Impact of the substructure description 9

3.2 Comparison of the DM subhalo models to the known Milky Way satellites 12

3.3 Subhalo angular power spectrum 13

4 Revisiting dark halo searches with Fermi-LAT $\quad 17$

5 CTA sensitivity to dark clumps $\quad 19$

5.1 Observation setup and nominal sensitivity 20

$\begin{array}{lll}5.2 & \text { Diffuse } \gamma \text {-rays and residual background } & 20\end{array}$

$\begin{array}{lll}5.3 & \text { Characteristics of the brightest halos } & 21\end{array}$

5.4 Likelihood ratio and test statistic (TS) 24

5.5 Sensitivity to dark clumps and comparison to other targets 24

6 Summary and discussion $\quad 26$

A Halo overdensity definition $\Delta$ and cosmology $\quad 29$

B Mean and variance from analytical approximations $\quad 30$

B.1 Power-law source count distribution 30

B.2 Mean and median of the brightest DM halo 30

B.3 APS mean, median, and variance $\quad 31$

$\begin{array}{lll}\text { C } & \text { APS convergence } & 33\end{array}$

D Virial mass and and $J$-factor for Milky Way satellites 34

E Details of the CTA analysis $\quad 35$

E.1 Observation strategy 36

$\begin{array}{lll}\text { E.2 Pre- and post-trial sensitivity } & 38\end{array}$

$\begin{array}{lll}\text { E.3 Likelihood } & 38\end{array}$

E.4 Distribution and values of the test statistic (TS) 39

\section{Introduction}

Dark matter (DM) indirect detection in $\gamma$-rays was first discussed almost 40 years ago $[1,2]$ in the context of the diffuse $\gamma$-ray emission. The most promising DM candidate is a weakly interacting massive particle (WIMP), which produces secondary $\gamma$-rays originating from the 
particles' self-annihilation [3]. As the signal from self-annihilating DM roughly goes as the DM density squared divided by the distance squared, the most promising targets result from an interplay between being close and/or massive, highly concentrated DM reservoirs. This made the Galactic centre (GC) a prime target, as first considered in [4]. However, it was soon recognised that setting constraints on DM would be limited by astrophysical background at $\mathrm{GeV}$ energies [5]. Higher energies face similar challenges, as illustrated by the first H.E.S.S. observations of the GC [6], and following DM interpretations [7]. Owing to their potentially high DM densities and small astrophysical backgrounds, dwarf spheroidal (dSph) galaxies orbiting the Milky Way [8] and dark clumps [9, 10] were proposed next. In particular, the question of the DM clump population boost of the Galactic signal $[11,12]$ and/or the detectability of individual clumps with future $\gamma$-ray satellites and ground-based instruments [12-14] was raised.

Since these pioneering studies, a steady progress has been made in estimating the prospects of DM indirect detection (or in setting constraints from non-detection) with the $\gamma$-ray sky. The launch of the Fermi $\gamma$-ray Space Telescope in 2008 with its main instrument, the Large Area Telescope (LAT) [15], was a huge step forward in exploring with unprecedented sensitivity the DM parameter space (see [16] for a review). Thanks to the all-sky survey observing strategy, the Fermi-LAT collaboration has set constraints on $\gamma$-ray lines [17-20] or excesses in the $\gamma$-ray continuum spectrum of the diffuse Galactic and extragalactic emission [21-23], galaxy clusters [24, 25], dSph galaxies [26-31], and dark clumps [28]. For dSph galaxies, whose known number is still growing from recent optical surveys, a stacking strategy allowed to exclude a thermal relic annihilation cross-section $\langle\sigma v\rangle$ of a few times $10^{-26} \mathrm{~cm}^{3} \mathrm{~s}^{-1}$ for WIMP masses $m_{\chi}<100 \mathrm{GeV}$ [30, 31]. Constraints from ground-based Cherenkov telescopes are competitive with Fermi-LAT limits to constrain DM particle masses above a few hundreds of GeV. These pointed instruments use a different observation strategy: the best constraints are obtained from DM searches in regions around the GC, leading to limits of $\langle\sigma v\rangle \lesssim 3 \times 10^{-24} \mathrm{~cm}^{3} \mathrm{~s}^{-1}$ for $m_{\chi} \sim \mathrm{TeV}[32,33]$; for dSph galaxies, the constraints either come from a stacking analysis (VERITAS [34, 35] and H.E.S.S. [36]) or from a deep observation of a single highly ranked object (MAGIC [37, 38]), leading to limits of $\langle\sigma v\rangle \lesssim 1-3 \times 10^{-24} \mathrm{~cm}^{3} \mathrm{~s}^{-1}$.

To take full advantage of this wealth of data and to set the most reliable and effective limits on DM, the modelling of the DM signal received as much attention. Beside the complex GC region (see, e.g., the Fermi-LAT analysis [39]), improvements were made in the DM modelling of dSph galaxies [40, 41], galaxy clusters [42-46], and diffuse emissions (see [47] for a recent review). To increase the sensitivity to DM indirect detection, multiwavelength analyses $[48,49]$ and observations of dSph galaxies [50-52] and galaxy clusters [53, 54] have been carried out. Enhancements of the signal-to-noise ratio arise from a better characterisation of $\gamma$-ray anisotropies and cross-correlation with other tracers (e.g., [55]). Note that most of these calculations at both the Galactic and extragalactic level depend on the hierarchical structure formation and on the survival and distribution of subhalos in their host halos. In particular, the detectability of dark clumps observed as $\gamma$-ray point sources with no counterparts at other wavelengths or from their imprint in the angular power spectrum (APS) over the diffuse DM background (that can be boosted by these same micro-halos), depends crucially on the modelling of these clumps. Their properties are investigated by heavy numerical simulations [56]. If state-of-the-art Milky Way like simulated halos, such as Via Lactea [57], Aquarius [58], and more recently COCO [59] reach a mass resolution of a few $10^{5}$ solar masses, this must be compared with the very uncertain minimal mass of the subhalos (set 
by the DM candidate couplings) that could be ten orders of magnitude below (e.g., [60]). Numerical simulations of the early universe have confirmed that such subhalos might survive until today [61], a result also deduced from theoretical calculations (see [62] for a review).

This paper revisits the question of the detectability of dark clumps for present and future $\gamma$-ray instruments. The prospects for the Fermi-LAT instrument were discussed by many authors in the light of high resolution numerical simulations [63-67], or based on semi-analytical approaches which extrapolate the clump population down to the mass of the smallest micro-halos [68-73]. Whereas recent efforts are turned towards a better discrimination between astrophysical or DM signals in sources with no counterparts in the Fermi-LAT catalogue [74-79], the constraints that can be set depend ultimately on the number of expected DM clumps, which is still disputed [80,81]. The sensitivity to dark clumps for the future Cherenkov Telescope Array (CTA) [82, 83] is discussed in [84], but a more up-to-date estimate is made here based on the foreseen CTA extragalactic survey [85]. We use the CLUMPY code $[86,87]$ to evaluate the impact of the clump distribution uncertainties on the ensuing $\gamma$-ray signal. Hundreds of Monte Carlo (MC) realisations are run per configuration to estimate the resulting uncertainties on the number of clumps, and to characterise the typical mass and distance of these detectable clumps. We also use realistic instrument responses in a plausible large-sky survey scenario to assess the sensitivity of CTA to these clumps. This provides a complementary view of CTA capabilities against pointed targets that will be part of the CTA DM programme [88-92].

The paper is organised as follows: in section 2, we present our modelling approach for the DM distribution in the Galaxy, focusing in particular on seven physical parameters important to the Galactic substructure distribution; section 3 presents the resulting $\gamma$-ray flux from the different models and describes several cross checks to demonstrate the consistency of our modelling; in section 4, we use our findings to revisit the possibility that DM subhalos might be present in the 3FGL catalogue of Fermi-LAT; section 5 presents the sensitivity of the future CTA to detect DM subhalos within its planned extragalactic sky survey and we finally discuss and summarise our findings in section $6 .^{1}$. Throughout the paper, we denote the mean of quantities $Q$ with a bar, $\bar{Q}$, and median values with a tilde, $\widetilde{Q}$. Global properties of the Galactic host halo are denoted with capital letters (mass $M$, positions $R$ within the halo, distance $D$ to observer), and properties of individual subhalos with lowercase letters (mass $m$ at distance $d$, positions $r$ within the subhalo). When referring to the brightest subhalo, variables are indicated with an asterisk, $Q^{\star}$.

\section{Modelling the $\gamma$-ray-emission from Galactic DM subhalos}

To assess detection prospects of Galactic DM subhalos, we explore various parameter sets for the substructure density. The average total Galactic halo density is left unchanged.

\footnotetext{
${ }^{1}$ The article is followed by a detailed appendix. In appendix A, we shortly review different definitions of cosmological matter overdensities used throughout this paper. In appendix B, we present useful formulae to analytically describe power-law source count distributions, and we use these results for a convergence study of the angular power spectra in appendix C. In appendix D, we present the derivation and the mass and annihilation factor for the spectroscopically confirmed satellite galaxies in the Milky Way, including recently discovered objects. In appendix E, we provide additional details about the CTA analysis performed in section 5
} 


\subsection{Modelling approach}

We use the CLUMPY code ${ }^{2}$ to model the $\gamma$-ray emission from Galactic DM subhalos. We refer the reader to $[86,87]$ for an extensive description of the CLUMPY code features and validation. CLUMPY has been used previously to study DM annihilation and/or decay in dSph galaxies [93-99] and galaxy clusters [46, 100, 101]. For the purpose of this work, we mainly use CLUMPY in the so-called 'skymap mode' which allows the fast computation of full-sky maps of DM annihilation or decay signals. We focus on DM annihilation only, for which the expected DM differential $\gamma$-ray flux at energy $E$, in the direction $\vec{k}=(\psi, \vartheta)$ and per solid angle $\mathrm{d} \Omega$ reads

where

$$
\frac{\mathrm{d} \Phi}{\mathrm{d} E \mathrm{~d} \Omega}(E, \vec{k})=\frac{\mathrm{d} \Phi^{\mathrm{PP}}}{\mathrm{d} E}(E) \times \frac{\mathrm{d} J}{\mathrm{~d} \Omega}(\vec{k})
$$

$$
\frac{\mathrm{d} \Phi^{\mathrm{PP}}}{\mathrm{d} E}(E)=\frac{1}{4 \pi} \frac{\langle\sigma v\rangle}{m_{\chi}^{2} \delta} \sum_{f} \frac{\mathrm{d} N_{\gamma}^{f}}{\mathrm{~d} E} B_{f},
$$

and where the $J$-factor is generically written as

$$
J(\vec{k}, \Delta \Omega)=\int_{\Delta \Omega} \int_{\text {l.o.s }} \rho^{2} \mathrm{~d} l \mathrm{~d} \Omega=\int_{0}^{2 \pi} \int_{0}^{\theta_{\text {int }}} \int_{\text {l.o.s }} \rho^{2}(\vec{k} ; l, \theta, \phi) \mathrm{d} l \sin \theta \mathrm{d} \theta \mathrm{d} \phi .
$$

In these equations, $m_{\chi}$ is the mass of the DM particle $\chi,\langle\sigma v\rangle$ is the velocity-averaged annihilation cross-section, and $\mathrm{d} N_{\gamma}^{f} / \mathrm{d} E$ and $B_{f}$ correspond to the spectrum and branching ratio of annihilation channel $f$. The parameter $\delta$ is $\delta=2$ for a Majorana and $\delta=4$ for a Dirac particle, and we choose $\delta \equiv 2$ in the remainder of this paper. In CLUMPY, $m_{\chi},\langle\sigma v\rangle$, and $B_{f}$ are user-defined parameters, from which the $\gamma$-ray annihilation spectrum is calculated (based on the parametrisations of [102]). The DM density $\rho$ is integrated along the line of sight (l.o.s.), and up to a maximum angular distance $\theta_{\text {int }}$. The overall DM density can be written $\rho_{\text {tot }}=\rho_{\text {sm }}+\rho_{\text {subs }}$, where $\rho_{\text {sm }}$ corresponds to the smooth component, and $\rho_{\text {subs }}$ corresponds to the substructures of the Galactic DM halo.

Generating skymaps with CLUMPY starts from setting DM properties: smooth DM profile, spatial and mass distribution of Galactic substructures, halo mass-concentration relation, DM particle mass, and annihilation/decay channels. The computation has been optimised as to draw only subhalos that outshine the mean DM signal (set by a user-defined precision), leading to a decomposition of the substructure signal $J_{\text {subs }}^{\text {tot }}=J_{\text {drawn }}+\left\langle J_{\text {subs }}\right\rangle$ into two components: $J_{\text {drawn }}$ is the signal from the substructures drawn in a realisation of the skymaps, and $\left\langle J_{\text {subs }}\right\rangle$ is the average signal from all 'unresolved' halos ${ }^{3}$, i.e., faint subhalos whose intrinsic $J$-factors do not pass the threshold defined from the precision level required by the user. Additional levels of clustering within subhalos are also considered using this average description. We refer the reader to $[86,87]$ for details on the computation of these quantities. For the purpose of this work, suffice to say that the higher the precision requirement, the more halos are drawn and the smaller is the contribution of $\left\langle J_{\text {subs }}\right\rangle$. A convergence analysis with respect to the precision requirement is presented in appendix $\mathrm{C}$.

The flexibility of CLUMPY allows the user to easily explore various models and configurations, calibrated (but not limited) to the results of various $\Lambda$ CDM numerical simulations such as the Aquarius [58], Phoenix [103] or Via Lactea II (VL II) [104] simulations, as well as their hydrodynamical updates [105-107].

\footnotetext{
${ }^{2}$ http://lpsc.in2p3.fr/clumpy

${ }^{3}$ For legibility purpose, we define $\left\langle J_{\text {subs }}\right\rangle$ to be the sum of $\left\langle J_{\text {subs }}\right\rangle$ and $J_{\text {cross-prod }}$ as defined in [87].
} 


\subsection{Milky Way dark matter halo}

The total DM density profile $\bar{\rho}_{\text {tot }}$ of the Milky Way (MW) is modelled with an Einasto profile with a slope $\alpha_{\mathrm{E}}=0.17$ and scale radius $R_{\mathrm{S}}=15.14 \mathrm{kpc}$, as suggested by [108]. The normalisation of the profile is computed to satisfy $\rho_{\odot} \equiv \rho\left(R_{\odot}=8 \mathrm{kpc}\right)=0.4 \mathrm{GeV} \mathrm{cm}^{-3}$, as estimated by [109] (see also [110, 111] for higher estimates). Although $R_{\odot}$ and $\rho_{\odot}$ suffer from large uncertainties [112-114], their exact values are subdominant for the purpose of our study. All our calculations use a maximum radius $R_{\mathrm{MW}}=260 \mathrm{kpc}$ of the MW DM halo, yielding a total MW mass $M_{\mathrm{MW}}=1.1 \times 10^{12} \mathrm{M}_{\odot}$, in agreement with [115]. As discussed in [86], we define the average substructure density, $\bar{\rho}_{\text {subs }}=f_{\text {subs }} \times M_{\mathrm{MW}} \times \mathrm{d} P / \mathrm{d} V$, where $\mathrm{d} P / \mathrm{d} V$ is the spatial distribution of Galactic substructures, and $f_{\text {subs }}$ is the global fraction of the MW mass contained in subhalos. Given $\bar{\rho}_{\text {tot }}$ and $\bar{\rho}_{\text {subs }}$, the smooth MW profile then is defined as $\rho_{\text {sm }}=\bar{\rho}_{\text {tot }}-\bar{\rho}_{\text {subs }}$. At sufficient angular distance from the GC $\left(\theta \gtrsim 10^{\circ}\right)$, this smooth component results in a diffuse background flux, $J_{\mathrm{sm}}$, that is found negligible by at least 3 orders of magnitude when compared to the flux of resolved subhalos or to the residual background of CTA (see $\S 5.2$ ).

\subsection{Substructure properties and set of models}

Substructures in their host halo are characterised by their mass and spatial distribution, as well as the description of the DM distribution within each subhalo. In the $\Lambda$ CDM hierarchical structure formation scenario, small structures collapse first and then fall and merge into larger structures. Subject to tidal forces, the 'unevolved' initial substructure distribution turns into an 'evolved' population whose properties differ from those of field halos [116]. Semi-analytical models as well as numerical simulations have been used to characterise the properties of these substructures down (or extrapolated down) to the smallest mass scale. The spread in the various results yield significant uncertainties, e.g., on the cosmological annihilation signal [117].

To assess the detectability of dark clumps, we build sets of models by varying seven important properties of Galactic substructures, as described in table 1. For all these models, the threshold mass of the smallest and most massive subhalos are fixed to $m_{\text {min }}=10^{-6} \mathrm{M}_{\odot}$ [118] and $m_{\max }=0.01 M_{\text {tot }}$ [119] respectively: increasing $m_{\max }$ would slightly increase the median $J$-factor of the brightest subhalo, leaving unchanged our conclusions; decreasing $m_{\min }$ amounts to adding an extra-population of very low-mass halos that would contribute to the average $\left\langle J_{\text {subs }}\right\rangle$ component, the effect being dependent on the slope $\alpha_{m}$ of the mass distribution (see below). For the angular resolutions considered in our calculations, the brightest resolved objects always outshine the diffuse DM background emission from $J_{\mathrm{sm}}+$ $\left\langle J_{\text {subs }}\right\rangle$, moreover, for CTA, the diffuse background is dominated by the instrumental residual background and not by the $\left\langle J_{\text {subs }}\right\rangle$ component.

The parameters and the choices made for their variation are briefly mentioned hereafter and their consequences on the flux are discussed in section 3.

1. Subhalo inner profile. As seen in Eq. (2.2), the DM density profile is the fundamental ingredient to estimate the astrophysical part of the DM annihilation flux. When ignoring baryonic effects, DM substructures are characterised by cuspy profiles, with two standard parametrisations being the Navarro-Frenk-White (NFW) and Einasto descriptions $[120,121]$. For a given subhalo, we calculate the normalisation and scale radius of the DM inner profile by providing the subhalo mass, $m_{\mathrm{vir}}$, and its concentration $c_{\mathrm{vir}}\left(m_{\mathrm{vir}}, R\right) \equiv r_{\mathrm{vir}} / r_{\mathrm{s}}$, where $R$ is the distance from the GC, and $r_{\mathrm{vir}}, r_{\mathrm{s}}$ are the virial 


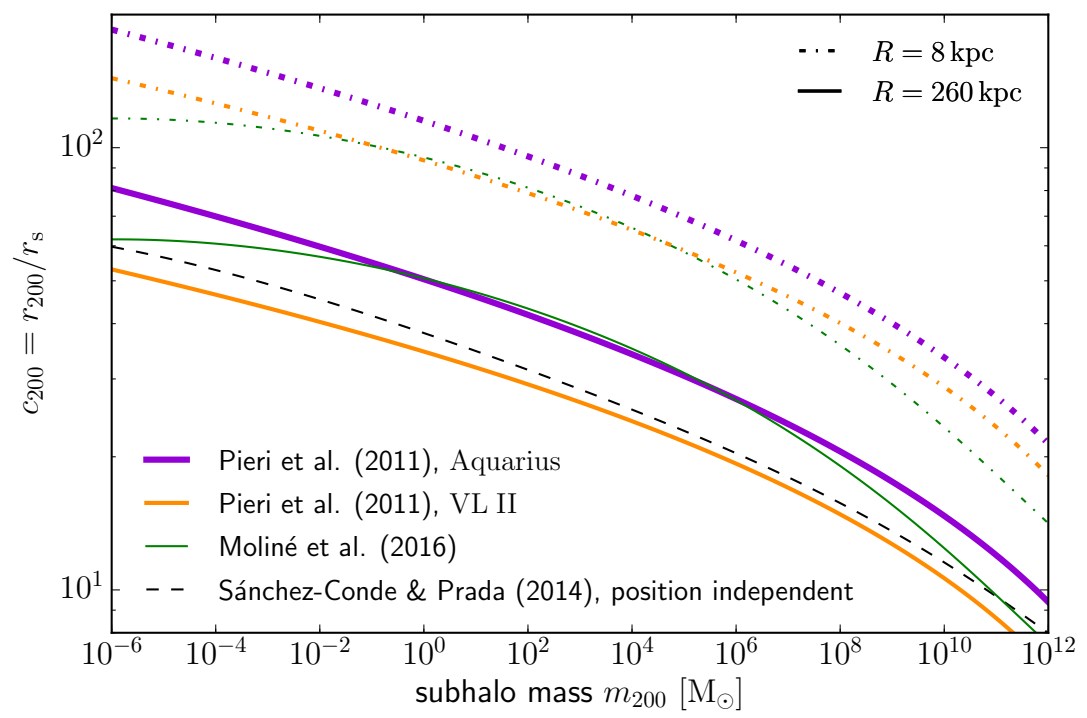

Figure 1: Models for the concentration $c_{200}$ of Galactic subhalos at redshift $z=0$. Beside the distance-independent parametrisation SP [126], shown are the values at the galactocentric distances $R=8 \mathrm{kpc}$ (dashed-dotted lines) and $R=260 \mathrm{kpc}$ (solid lines) from [67] (violet and orange) and [129] (green).

and scale radius of the subhalo, respectively. The meaning of $c_{\mathrm{vir}}$ is further discussed in the next paragraph. For the Einasto profile, the shape parameter $\alpha_{\mathrm{E}}$ introduces an additional degree of freedom, which we fix to $\alpha_{\mathrm{E}} \equiv 0.17 .^{4}$ An Einasto profile is the default configuration, and the effect of switching to NFW is performed in the VAR0 model (see table 1). Note that recent simulations have shown that halos close to the free streaming scale [122-125] could be cuspier than the NFW profile. Including in CLUMPY this mass-dependent inner slope -Eq. (2) of [124] - leads to a $13 \%$ to $43 \%$ increase of $\left\langle J_{\text {subs }}\right\rangle$ (comparable to the $12 \%$ to $67 \%$ increase found in [124]), but more importantly here, we checked that these boosted microhalos provide no detectable dark clumps.

2. Mass-concentration parametrisation $c(m)$. Once the parametrisation of a subhalo inner profile is chosen, its structural parameters (normalisation and scale radius) are fully determined from the mass $m_{\Delta}$ and concentration-mass $c_{\Delta}-m_{\Delta}$ relation (see appendix $\mathrm{A}$ for a definition of $\Delta=\operatorname{vir}, 200,500 \ldots$ ). The latter depends on the subhalo evolution in its host halo, i.e. its location and 'evolved' mass. Several parametrisations, based on the results of numerical simulations have been proposed in the last few years, the most recent suggesting a flattening of the relation at low masses [126] and a higher concentration of subhalos compared to field halos. The latter effect was shown to yield an extra $\sim 5$ boost factor [127-129] on $\left\langle J_{\text {subs }}\right\rangle$ compared to previous calculations. Here, we consider the field halo Sánchez-Conde \& Prada [126] parametrisation (SP), the distance-dependent Pieri et al. [67] parametrisations (P-VLII based on the Via Lactea II simulation and P-Aq based on Aquarius) and the Moliné et al. [129] parametrisation. ${ }^{5}$

\footnotetext{
${ }^{4}$ We also investigated the impact of a scattering in $\alpha_{\mathrm{E}}$. The authors of [58] find the Aq-A subhalos equally well described by Einasto profiles with $0.16 \leq \alpha_{\mathrm{E}} \leq 0.20$. For $\alpha_{E}=0.16$, the $J$-factors increase by $\lesssim 20 \%$ compared to $\alpha_{E}=0.17$, and decrease by $\lesssim 60 \%$ when choosing $\alpha_{E}=0.20$. The DM constraints set from the brightest subhalo change by the same amount, whereas the effect on $\left\langle J_{\text {subs }}\right\rangle$ is subdominant.

${ }^{5}$ As for several other refinements discussed in this section, the Pieri et al.and Moliné et al.parametrisations
} 
These mass-concentration prescriptions are displayed in figure 1. The Pieri et al. and Moliné et al. approaches account for the fact that the closer a subhalo of a given mass is to the GC, the more concentrated it is. The various parametrisations are compared at a distance of $8 \mathrm{kpc}$ from the GC (dotted-dashed lines), where the P-VLII and Moliné et al. parametrisations produce similar concentrations, while P-Aq appears systematically higher. At large distance from the GC (solid lines), all parametrisations yield lower concentrations that become more compatible with the SP field halo distanceindependent parametrisation (dashed line). In the following, we use the SP description as our conservative baseline and investigate the distance-dependent effects of the P-VLII and Moliné parametrisations in both the VAR6 and HIGH models. We will discard the $\mathrm{P}$-Aq prescription in the following, but remind that using this parametrisation would result into even larger $J$-factors than used in our optimistic model HIGH. ${ }^{6}$

3. Width of the mass-concentration distribution, $\sigma_{c}$. Rather than assuming a single concentration for a given halo mass from the mean parametrisations above, the concentration is drawn from a log-normal distribution of width $\sigma_{c}$ around the values given by these parametrisations. This is incorporated to account for the intrinsic scatter of the $c(m)$ relation found in numerical simulations. We consider $\sigma_{c}=0.14$ as our default value $[126,130]$, and study the impact of a larger scatter in the VAR2 model where $\sigma_{c}=0.24[131]$ is used.

4. Number of halos $N_{\text {calib }}$ between $10^{8}$ and $10^{10} \mathrm{M}_{\odot}$. This number is used as a calibration for the total number of subhalos. $\Lambda$ CDM simulations of MW size halos predict an overabundance of high-mass subhalos compared to the currently known satellite galaxies; this is the so-called 'missing satellites' problem [132], which is linked to the 'too-big-tofail' problem [133]. Baryonic feedback onto the cusps of DM subhalos could possibly solve this tension [134, 135]. Indeed, hydrodynamical simulations roughly show half as many high-mass subhalos as DM only simulations. About $100-150$ objects are obtained above $10^{8} \mathrm{M}_{\odot}[105,106]$, and we choose $N_{\text {calib }}=150$ as our default value. A more subhalo-rich configuration, used in models VAR4 and HIGH (see table 1), is defined by $N_{\text {calib }}=300$ as motivated by the results of DM-only simulations [58].

5. Index of the clump mass distribution, $\alpha_{m}$. Numerical simulations show that the DM halo mass distribution is well described as $\mathrm{d} N / \mathrm{d} m \propto m^{-\alpha_{m}}$. For the reference model we take $\alpha_{m}=1.9$ as suggested by numerical simulations of MW-like halos [58, 104] and investigate a slightly steeper mass function in the VAR1 model, where $\alpha_{m}=2.0$. Together with $N_{\text {calib }}, m_{\min }$, and $m_{\max }$, the choice of $\alpha_{m}$ determines the total number of clumps $N_{\text {tot }}$ and their total mass $M_{\text {subs }}$ (see [86] for details). In table 1, we provide the resulting $N_{\text {tot }}$ and, with the knowledge of the total mass of the Galaxy, $M_{\mathrm{MW}}$, the global portion of DM bound into subhalos, $f_{\text {subs }}=M_{\text {subs }} / M_{\mathrm{MW}}$.

6. Spatial distribution of Galactic substructures, $\mathrm{d} P / \mathrm{d} V=\bar{\rho}_{\text {subs }} / M_{\text {subs }}$. The fraction of mass bound into substructures is expected to decrease towards the GC, as subhalos are tidally disrupted by the strong gradient of the gravitational potential. This is discussed in detail in [116], where the authors argue that this effect is the result of a

have been implemented in CLUMPY for the purpose of this work, but are not yet available in the public version.

${ }^{6}$ We also investigated the P-Aq model, and found a $\sim 60 \%$ increase for the flux from the brightest subhalos compared to the P-VLII description. This increase would also improve the sensitivities presented in figure 13 by the corresponding factor. 


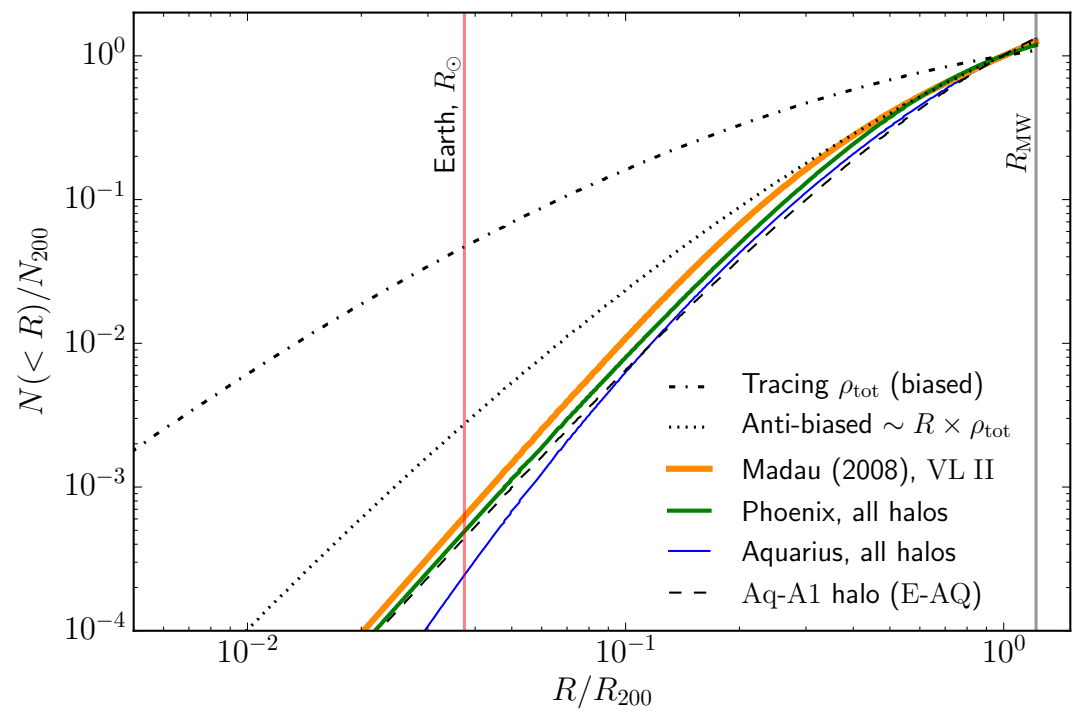

Figure 2: Number of subhalos within $R$, normalized to $N_{200}$, the number of subhalos within $R_{200}$, up to $R_{\mathrm{MW}} / R_{200}=1.22\left(R_{200}=213.5 \mathrm{kpc}\right.$ for our Galactic halo $)$. We also show the position of the observer at $R_{\odot} / R_{200}=0.037$. The number of subhalos, $N(R)$, results from the subhalo number density $\mathrm{d} N / \mathrm{d} V=N_{\text {tot }} \cdot \mathrm{d} P / \mathrm{d} V$.

selection effect of the 'evolved' subhalos (suffering from tidal stripping), the 'unevolved' distribution following the host smooth distribution. Figure 2 displays the cumulative number of halos as a function of the distance to the GC, where $\bar{\rho}_{\text {subs }} \propto \bar{\rho}_{\text {tot }}$ is shown as dash-dotted line. To correctly describe $\bar{\rho}_{\text {subs }}$, a generic 'anti-biased' parametrisation $\bar{\rho}_{\text {subs }} / \bar{\rho}_{\text {tot }} \propto R$ was proposed in $[136,137]$ (dotted line). This is to be compared to the result obtained from numerical simulations, namely, a fit to Aq-A1 (well described by an Einasto profile 'E-AQ', long-dashed line) or to all Aquarius halos (curved power-law, cyan) halos [58], or subhalos of the Via Lactea II simulation [104, 138] (orange). We also show the profile for the Phoenix simulation of galaxy clusters (rescaled to $R_{200}$, green line) described by an Einasto profile [103], which leads to a comparable dependence. Simulations produce slopes steeper than $\bar{\rho}_{\text {subs }} / \bar{\rho}_{\text {tot }} \propto R$, with the Aquarius and Phoenix results found to be well described by $\bar{\rho}_{\text {subs }} / \bar{\rho}_{\text {tot }} \propto R^{1.3}$ in [116]'s model. In table 1 , we choose the Einasto profile fitted to the Aquarius A-1 halo as our default setup and use the Via Lactea II parametrisation in the VAR3 and HIGH models. Table 1 also gives the local DM mass fraction under the form of subhalos in the solar neighbourhood, $f_{\text {subs }}\left(R_{\odot}\right)=\bar{\rho}_{\text {subs }}\left(R_{\odot}\right) / \bar{\rho}_{\text {tot }}\left(R_{\odot}\right)$, which is dependent on the chosen $\bar{\rho}_{\text {subs }}$ profile.

7. Flag for sub-substructures. The default calculation assumes no further substructures within Galactic subhalos. However, several levels of substructures (clumps within clumps) can be taken into account by CLUMPY for the flux calculation, which would boost the signal further. This is tested in model VAR5, where we assume self-similarity, with respect to the model LOW, between subhalos and sub-subhalos (following what has been shown by both the Aquarius and Via Lactea II simulations [64, 139]), i.e. $f_{\text {subsub }}=0.19, \mathrm{E}-\mathrm{AQ}$ for the spatial distribution of sub-subhalos within their host, and SP for the $c(m)$ parametrisation. 


\begin{tabular}{|c|c|c|c|c|c|c|c|c|c|c|c|}
\hline & Model & VAR0 & LOW & VAR1 & VAR2 & VAR3 & VAR4 & VAR5 & VAR6a & VAR6b & HIGH \\
\hline \multirow{7}{*}{ 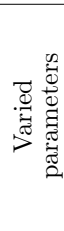 } & \multirow{7}{*}{$\begin{array}{c}\text { inner profile } \\
\alpha_{m} \\
\sigma_{c} \\
\bar{\varrho}_{\text {subs }} \\
N_{\text {calib }} \\
\text { sub-subhalos? } \\
c(m)\end{array}$} & NFW & $\mathrm{E}$ & $\mathrm{E}$ & $\mathrm{E}$ & $\mathrm{E}$ & $\mathrm{E}$ & $\mathrm{E}$ & $\mathrm{E}$ & $\mathrm{E}$ & $\mathrm{E}$ \\
\hline & & 1.9 & 1.9 & 2.0 & 1.9 & 1.9 & 1.9 & 1.9 & 1.9 & 1.9 & 1.9 \\
\hline & & 0.14 & 0.14 & 0.14 & 0.24 & 0.14 & 0.14 & 0.14 & 0.14 & 0.14 & 0.14 \\
\hline & & E-AQ & E-AQ & E-AQ & E-AQ & M-VLII & E-AQ & E-AQ & E-AQ & E-AQ & M-VLII \\
\hline & & 150 & 150 & 150 & 150 & 150 & 300 & 150 & 150 & 150 & 300 \\
\hline & & no & no & no & no & no & no & yes & no & no & no \\
\hline & & SP & SP & $\mathrm{SP}$ & $\mathrm{SP}$ & $\mathrm{SP}$ & $\mathrm{SP}$ & $\mathrm{SP}$ & Moliné & P-VLII & P-VLII \\
\hline \multirow{6}{*}{ 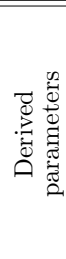 } & $\bar{c} N_{\text {tot }}\left(\times 10^{14}\right)$ & 6.1 & 6.1 & 150 & 6.1 & 6.1 & 12 & "6.1 & 6.1 & 6.1 & 12 \\
\hline & $f_{\text {subs }}[\%]$ & 19 & 19 & 49 & 19 & 19 & 38 & 19 & 19 & 19 & 38 \\
\hline & $f_{\text {subs }}\left(R_{\odot}\right)[\%]$ & 0.30 & 0.30 & 0.77 & 0.30 & 0.47 & 0.59 & 0.30 & 0.30 & 0.30 & 0.93 \\
\hline & $\widetilde{D}_{\mathrm{obs}}^{\star}[\mathrm{kpc}]$ & $22_{-16}^{+32}$ & $19_{-14}^{+27}$ & $13_{-10}^{+27}$ & $21_{-15}^{+33}$ & $20_{-15}^{+22}$ & $17_{-13}^{+26}$ & $21_{-14}^{+30}$ & $8_{-6}^{+18}$ & $9_{-6}^{+14}$ & $8_{-6}^{+11}$ \\
\hline & $\log _{10}\left(\widetilde{m}_{\text {vir }}^{\star} / \mathrm{M}_{\odot}\right)$ & $9.0_{-1.4}^{+0.8}$ & $8.8_{-1.4}^{+0.8}$ & $8.5_{-1.5}^{+0.9}$ & $8.9_{-1.4}^{+0.8}$ & $9.0_{-1.3}^{+0.7}$ & $8.9_{-1.4}^{+0.9}$ & $9.0_{-1.4}^{+0.7}$ & $7.9_{-1.6}^{+1.4}$ & $7.9_{-1.5}^{+1.4}$ & $8.2_{-1.5}^{+1.2}$ \\
\hline & $\log _{10}\left(\frac{\widetilde{J}^{\star}}{\mathrm{GeV}^{2} \mathrm{~cm}^{-5}}\right)$ & $19.9_{-0.3}^{+0.4}$ & $20.0_{-0.3}^{+0.5}$ & $20.0_{-0.3}^{+0.4}$ & $20.0_{-0.3}^{+0.4}$ & $20.1_{-0.3}^{+0.4}$ & $20.2_{-0.3}^{+0.4}$ & $20.3_{-0.3}^{+0.5}$ & $20.3_{-0.4}^{+0.5}$ & $20.4_{-0.3}^{+0.5}$ & $20.8_{-0.4}^{+0.5}$ \\
\hline
\end{tabular}

Table 1: Parameters for the different models investigated in this study. The first seven lines correspond (from top to bottom) to: the subhalo density profile, the slope of the subhalo mass distribution, the width of the concentration distribution, the subhalo spatial distribution, the number of objects between $10^{8}$ and $10^{10} \mathrm{M}_{\odot}$, the flag for sub-subhalos, and the mass-concentration relation. The columns are ordered by increasing flux of the brightest object. 'NFW' stands for a Navarro-Frenk-White profile and 'E' for an Einasto profile with $\alpha_{\mathrm{E}}=0.17$. 'E-AQ' is the Einasto parametrisation fitted to the substructure distribution in Aquarius simulation [58], while 'M-VLII' corresponds to the Via Lactea II parametrisation of Madau et al. [104]. The mass concentration relation is 'SP' for the Sánchez-Conde \& Prada parametrisation [126], or the distance-dependent description by Moliné et al. [129], respectively by Pieri et al. [67], 'P-VLII'. Derived parameters in the six bottom rows are the following: $N_{\text {tot }}$ is the total number of subhalos in the MW; $f_{\text {subs }}$ is the global mass fraction contained in subhalos; $f_{\text {subs }}\left(R_{\odot}\right)$ is the mass fraction contained in subhalos at the solar distance from the GC; $\widetilde{D}_{\text {obs }}^{\star}, \widetilde{m}_{\text {vir }}^{\star}$, and $\widetilde{J}^{\star}$ are the median distance from the observer, mass, and $J$-factor of the brightest subhalo from the 500 realisations of each model.

\section{Global properties of the models}

Before performing analyses dedicated to Fermi-LAT and CTA in section 4 and section 5, we describe here the overall behaviour of the models with respect to the substructure properties described above. This is done by the inter-comparison of the various models ( $\S 3.1$ ), by comparison to the known MW satellites ( $\S 3.2$ ), and by comparison of the angular power spectrum to previous calculations $(\S 3.3)$.

\subsection{Impact of the substructure description}

Each substructure property that is varied according to table 1 essentially impacts the number of substructures and/or their associated $J$-factors. Five hundred skymap realisations for each modelling (LOW, HIGH, VAR0 to VAR6) have been simulated. The bottom half of table 1 gives the global properties of each modelling, averaged over the 500 realisations. Compared to the LOW model, only the calibration number $N_{\text {calib }}$ and the slope of the mass distribution affect the total number of subhalos. However, the number of halos within a given $J$-factor range will depend on all substructure-related properties and one may therefore use histograms of the subhalos $J$-factors to assess the importance of each property.

For a given model $X$, we define $\bar{N}_{X}(>J)$ as the mean number of halos with a $J$-factor above a certain threshold $J$, averaged over the 500 realisations. This quantity is shown in the top panel of figure 3 for model LOW (pale blue) and HIGH (red). The behaviour of the histograms is well approximated by a power-law $\propto J^{1-\alpha}$, with $\alpha \sim 2$ over a large range of 


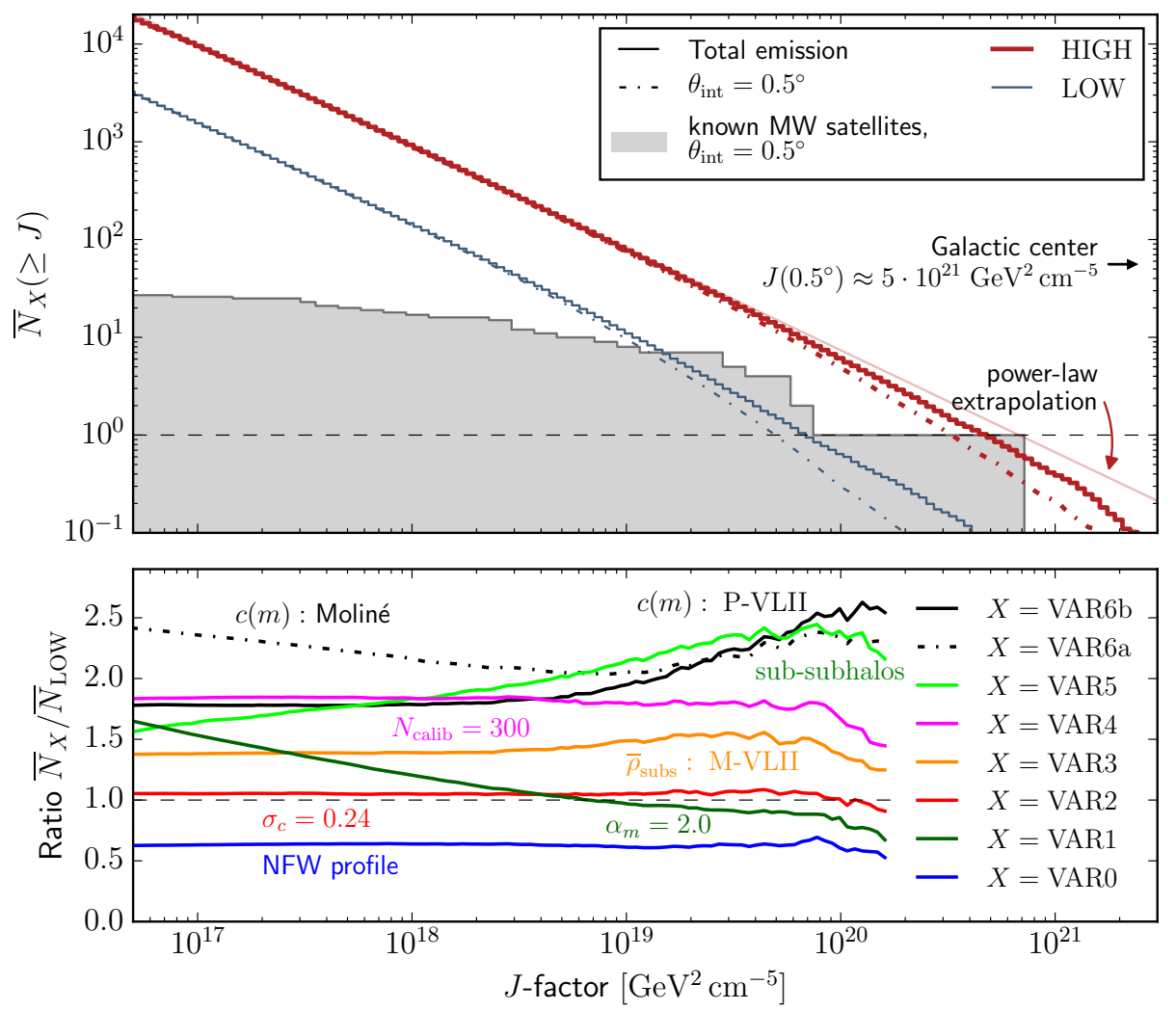

Figure 3: Top: cumulative source count distribution of Galactic subhalos (full sky, averaged over 500 simulations) for model LOW and HIGH. The solid lines show the distribution of the total $J$-factors, $J\left(\theta_{\text {vir }}\right)$, the dashed-dotted lines the distribution when only taking into account the emission from the central $0.5^{\circ}$ of the subhalos, $J\left(0.5^{\circ}\right)$. The grey-shaded histogram shows the cumulative distribution of all known dSph galaxy objects, including the Large and Small Magellanic cloud, with the values listed in table 4. Bottom: ratio of all VAR $i$ models to LOW.

$J$ values (thin solid red line). We also define the median $J$-factor of the brightest halo in a given model, $\widetilde{J}^{\star}$, and report these values in table 1 , along with the median mass, $\widetilde{m}_{\text {vir }}^{\star}$, and distance from the observer, $\widetilde{D}_{\text {obs }}^{\star}$, of this brightest halo. We refer the reader to appendix B for a detailed discussion of the $J^{\star}$ distribution and how $\widetilde{J}^{\star}$ and $\bar{N}(>J)$ are connected.

The ratio $N_{X}(>J) / N_{\mathrm{LOW}}(>J)$ is plotted in the bottom panel of figure 3 , where $X=\operatorname{VAR} i, \quad i \in[0,6]$ :

- Changing the subhalo inner profile (VAR0, blue), the substructure spatial distribution (VAR3, orange), the normalization of the mass distribution (VAR4, magenta), or the width of the mass-concentration description (VAR2, red) yields an increase or decrease of the number of clumps, uniformly over the entire $J$-factor range.

- Changing the subhalo inner profile or changing the subhalo spatial distribution yields a $\sim 40 \%$ change compared to the LOW model, while the width of the concentration distribution only affects the result by a few percents. The other substructure properties do not affect the number of subhalos in the same way for low and high $J$-factor values. 


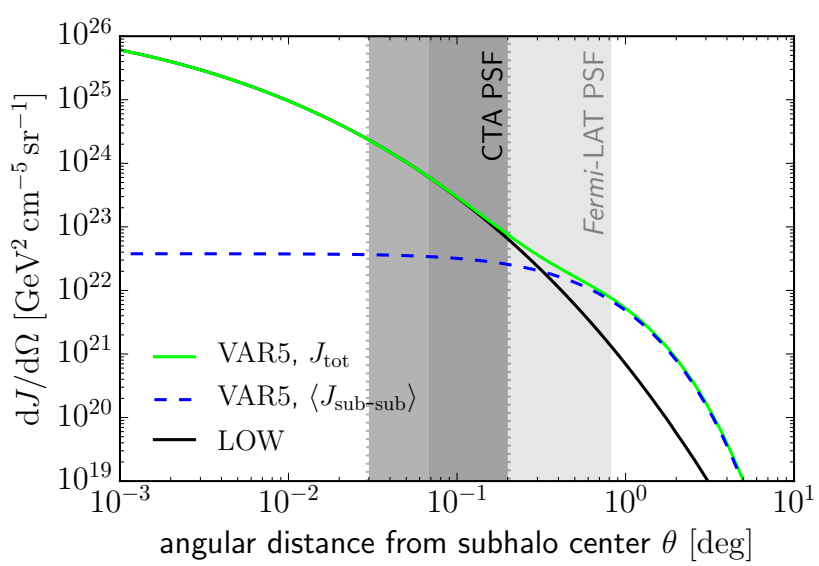

Figure 4: The influence of sub-subhalos as a function of the integration angle. For typical bright detectable DM halos (see table 3), the signal with sub-substructures (green solid line) is compared to the signal without them (black solid line). The angular resolutions (68\% containment radius; "point spread function", PSF) for CTA [83] at $E_{\gamma} \geq 30 \mathrm{GeV}$ and Fermi-LAT at $E_{\gamma} \geq 1 \mathrm{GeV}[15,140]$ are overlaid (grey shaded areas). For CTA, the sub-substructures contribution is negligible.

- Including a boost from sub-subhalos (VAR5, light green) or having a distance-dependent concentration prescription (VAR6, black) both produce a similar effect: a larger number of halos in each $J$-factor bin, the effect increasing with increasing $J$ (from $\sim 50-70 \%$ to more than a factor 2).

- For the highest $J$-decade, both distant-dependent concentration prescriptions VAR6a and VAR6b result in compatible $J$-factors. This is well understood given that the high$J$ end is populated by subhalos close to us, i.e. also close to the GC with $R \approx 10 \mathrm{kpc}$. At these distances, P-VLII and Moliné concentrations are in agreement on a wide mass range (figure 1). The prescriptions then differ for less luminous subhalos, which represent subhalos at larger galactocentric distances. For those objects, Moliné et al. predict larger concentrations, which results into brighter objects and a steepening of the source count distribution.

- Steepening the slope of the mass function (VAR1, dark green) increases (decreases) the number of faint (bright) halos, the amplitude of the effect remaining $\lesssim 50 \%$ for the $\sim 10^{3}$ most luminous subhalos.

Including sub-subhalos is only significant in the outskirts of the halos. This is shown in figure 4 , where $\mathrm{d} J / \mathrm{d} \Omega$ is plotted as a function from the distance to the centre for the LOW (grey solid line) and VAR5 (dashed blue and green solid lines) models. This finding is in agreement with [139]. For the angular resolution of the background-dominated CTA (grey band), however, they do not play a significant role and we therefore do not include them in the remainder of this study. ${ }^{7}$

From the range of substructures properties tested as deviations from the LOW model, we find the distance-dependent concentration parametrisation and boost from sub-subhalos to have the larger effect in terms of the number of halos with the largest $J$-factors. For

\footnotetext{
${ }^{7}$ Assuming a spatial dependence of the sub-subhalo concentration - analogous to model VAR5 on the subsub level-possibly increases this contribution within the CTA resolution. However, the boost at this second level of substructures is very uncertain and could even be negligible for these objects [129].
} 


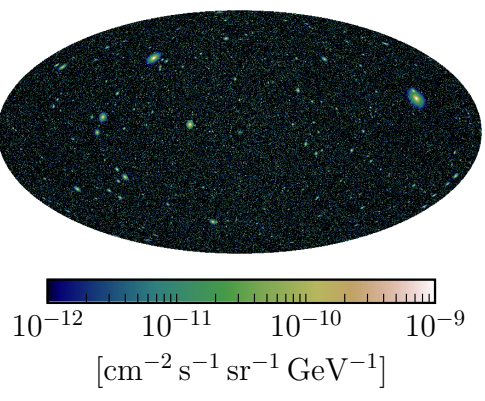

$$
\left[\mathrm{cm}^{-2} \mathrm{~s}^{-1} \mathrm{sr}^{-1} \mathrm{GeV}^{-1}\right]
$$

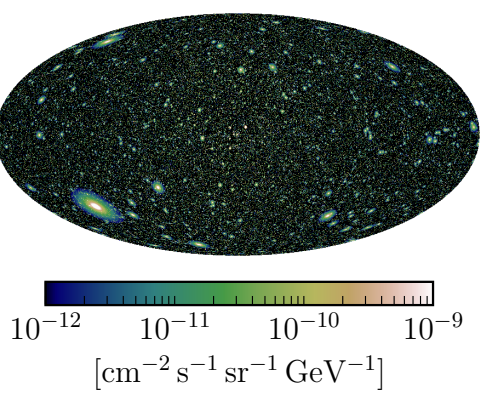

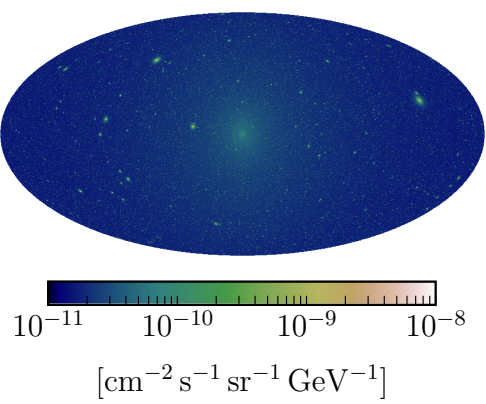

(a) Model LOW

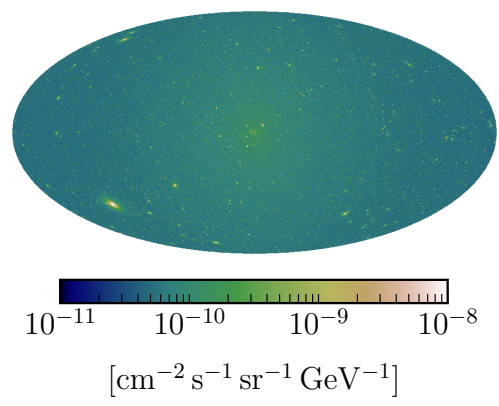

(b) Model HIGH
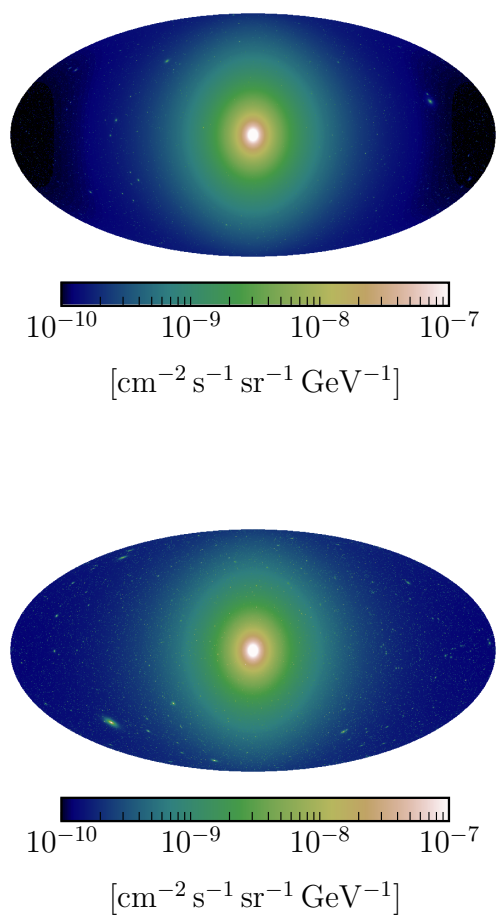

Figure 5: One statistical realisation of the Galactic differential intensity at $4 \mathrm{GeV}$, for the models LOW and HIGH. In the left column, only the flux from the resolved substructures is shown $\left(J_{\text {drawn }}\right)$. In the middle column, the flux from all substructures, resolved and unresolved, is shown $\left(J_{\text {drawn }}+\left\langle J_{\text {subs }}\right\rangle\right)$. In the right column, the total Galactic emission is shown ( $J_{\mathrm{sm}}$ additionally included). Note the different colour scales between the columns. The particle physics term is computed from a thermal relic cross-section, $m_{\chi}=200 \mathrm{GeV}$, and $\chi \chi \rightarrow b \bar{b}$. The maps are drawn with a HEALPix resolution $N_{\text {side }}=512$.

CTA-like angular resolutions, we conclude that the mass-concentration relation is the most important substructure property to pin down in order to make reliable detectability studies.

We use the distance-dependent concentration P-VLII by Pieri et al. in the HIGH model and, unless stated otherwise, the remaining of the paper will use the HIGH model as an optimistic template, while LOW remains default. For illustration purpose, we display in figure 5 the two corresponding differential flux skymaps computed at $4 \mathrm{GeV}$. The flux is obtained assuming a $200 \mathrm{GeV}$ DM candidate which annihilates exclusively in the $b \bar{b}$ channel. The left column shows maps of the substructures drawn by CLUMPY, while the middle column displays the total (resolved+unresolved) substructure contribution. As discussed above, more subhalos are resolved in model HIGH, and the flux of the unresolved component is also higher. The right column displays the total flux in both cases, i.e. including the smooth Galactic halo component, which is the dominant component towards the GC.

\subsection{Comparison of the DM subhalo models to the known Milky Way satellites}

More than twenty dSph galaxies are known to orbit the Milky Way. Formed from the most massive DM subhalos, these objects are prime targets for indirect detection as their DM 
content, and therefore $J$-factors can been inferred from stellar kinematics, e.g. [94, 96]. The mass and $J$-factors of these objects are discussed in appendix D, table 4 summarises their main properties. These values are used below for a sanity check of our models.

First, the grey shaded area in the top panel of figure 3 corresponds to the cumulative histogram of $J$-factors built from the known dSph galaxies, plus the SMC, and the LMC. The $J$-factors are reported within an integration angle of $\theta_{\text {int }}=0.5^{\circ}$, as benchmark angular resolution of Fermi-LAT [15, 140]. For display purposes, we have used the median values of the $J$-factors only, but we remind the reader that these values may be very uncertain for ultra-faint dSph galaxies. For lower $J$ values, the number of detected dSph galaxies becomes much lower than the number of subhalos measured from the models. This is what one would expect given that the most numerous low-mass halo would not have retained gas and formed stars to become identified as dSph galaxies [106]. The high- $J$ end of the histogram $\left(J \gtrsim 10^{19} \mathrm{GeV}^{2} \mathrm{~cm}^{-5}\right)$ lies between the LOW and HIGH models but for the very last bin. The latter corresponds to the recently-discovered Triangulum II galaxy, that we have tentatively analysed here. Note that the authors of [141], using a non-spherical halo hypothesis have reported a median $J\left(0.5^{\circ}\right)=1.6 \times 10^{20} \mathrm{GeV}^{2} \mathrm{~cm}^{-5}$ for Triangulum II, which would bring the last bin down to lie between LOW and HIGH. ${ }^{8}$ This behaviour of the MW satellites gives confidence to the fact that the LOW and HIGH models do indeed encompass the uncertainties surrounding the subhalo distribution.

Second, the mainly illustrative figure 6 gives the location of the detected MW satellites (red and orange dots) in the $J-m_{\text {vir }}$ (left) and $J-D_{\text {obs }}$ planes (right) on top of the distribution of subhalos of model HIGH. It shows that MW satellite galaxies probe, as expected, the high-mass and high-distance ends of the subhalo population. The horizontal dotted lines indicate how many subhalos are expected in a given realisation, and as above, model HIGH is in excess compared to the known objects. They could be yet-to-discover dSph galaxies or dark halos, and the distribution of entries (shaded blue scale) shows a preference for halos slightly less massive than the known dSph galaxies. Finally, the brightest subhalo expected for Fermi-LAT and CTA are given by the green and yellow stars, respectively. The Fermi-LAT subhalo is brighter because of the larger accessible survey area (see section 4 and section 5 for details).

\subsection{Subhalo angular power spectrum}

The angular power spectrum (APS) of the subhalo $\gamma$-ray sky maps is a powerful tool for DM analyses and provides another cross-check for our analysis. The APS $C_{\ell}$ of an intensity map $I(\vartheta, \varphi)$ is defined as

$$
C_{\ell}=\frac{1}{2 \ell+1} \sum_{m}\left|a_{\ell m}\right|^{2}
$$

with $a_{\ell m}$ the coefficients of the intensity map decomposed into spherical harmonics $Y_{\ell m}$,

$$
I(\vartheta, \varphi)=\sum_{\ell=0}^{\ell_{\max }} \sum_{m=-\ell}^{m=+\ell} a_{\ell m} Y_{\ell m}(\vartheta, \varphi) .
$$

CLUMPY's APS calculation relies on the HEALPix ${ }^{9}$ The median and variance of $C_{\ell}$ are calculated for each of the $500 J_{\text {drawn }}$ maps produced for all models, with HEALPix resolution

\footnotetext{
${ }^{8}$ When considering the credible intervals, the Triangulum II value of [141] and ours are nonetheless compatible within $1 \sigma$.

${ }^{9}$ http://healpix.sourceforge.net/ package [142].
} 


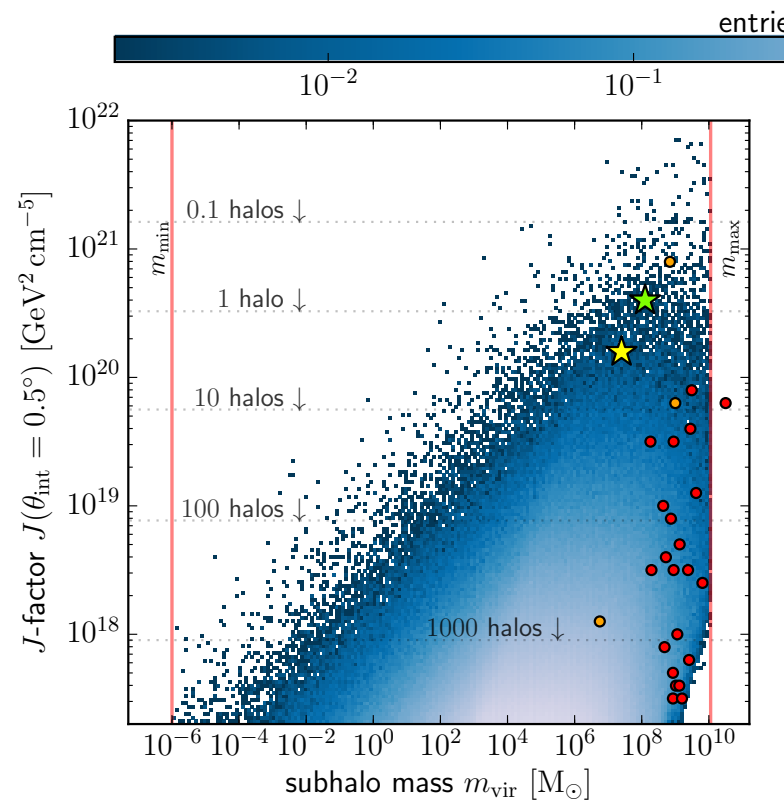

tries in bin

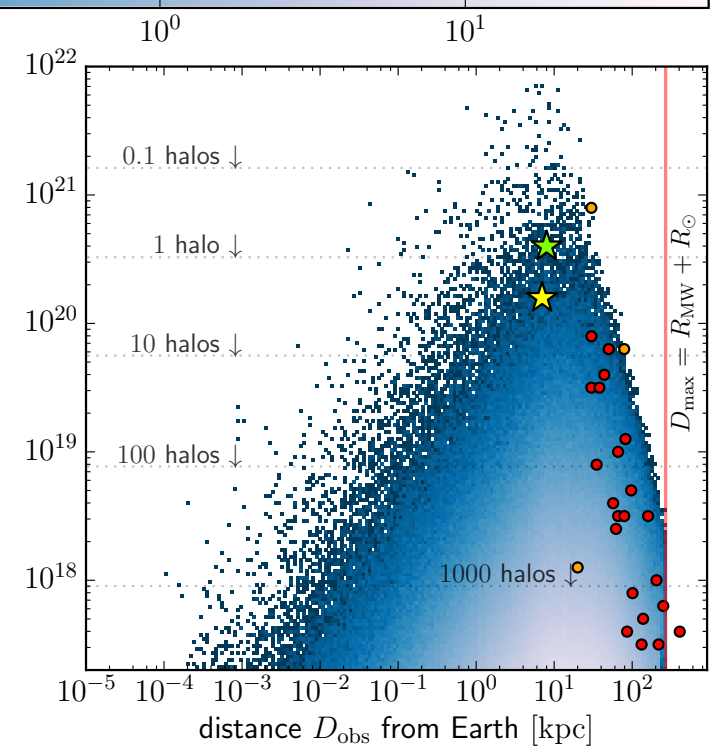

Figure 6: Relation between the brightness of the subhalos and their mass (left) or distance to the observer (right). The histograms are shown for the subhalo model HIGH and averaged over 500 simulations. The projection along the vertical axis gives the source count distribution $\langle\mathrm{d} N / \mathrm{d} J\rangle$. The dotted lines denote the integrated bins above the respective line, $\bar{N}_{\mathrm{HIGH}}(\geq J)$, identical to what is presented in figure 3. The known MW satellites listed in table 4 are displayed as red dots (and orange dots for the dSph discovered most recently). The green asterisk marks the median brightest subhalo expected for Fermi-LAT and the yellow asterisk for a dark subhalo search with CTA (see section 4 and section 5). In this figure, all $J$-values are calculated at $\theta_{\text {int }}=0.5^{\circ}$.

$N_{\text {side }}=4096$. The power $C_{\ell}$ caused by $J_{\text {drawn }}$ strongly depends on the number of simulated objects, and we show in the convergence study of appendix $\mathrm{C}$ that most of the power at all multipoles is generated by the $\mathcal{O}(100)$ brightest subhalos. ${ }^{10}$ As already underlined in previous analyses $[144,145]$, the subhalo power spectrum is Poisson-like in the range $\ell \lesssim 500$, and flattens at higher multipoles due to the extended size of the brightest (high-mass) subhalos (see figure 7). It is possible to connect the subhalo intensity APS to the source count distributions in figure 3. For point-like sources and at small angular scales, [144] showed that the angular power is approximately constant (Poisson-like) at all multipoles, and can be calculated by the one-subhalo term:

$$
C_{\ell} \approx C^{1 \mathrm{sh}} \approx \frac{1}{16 \pi^{2}} \int_{L_{\min }}^{L_{\max }} \int_{0}^{l_{\max }} \frac{L^{2}}{l^{2}} \frac{\mathrm{d} \bar{n}(l, L)}{d L} \mathrm{~d} l \mathrm{~d} L \equiv C_{\mathrm{P}}^{I},
$$

where $\bar{n}(l, L)$ denotes the number density of the subhalos, averaged over the solid angle $\Omega$. The coordinates $(l, \Omega)$ denote spherical coordinates with the observer at $l=0$, and $L$ is the total luminosity of a subhalo. For a point-like DM halo, $L=\frac{\langle\sigma v\rangle}{2 m_{\chi}^{2}} \frac{\mathrm{d} N}{\mathrm{~d} E} \mathcal{L}$, with the luminosity

\footnotetext{
${ }^{10}$ We showed in [87] that the contribution of the unresolved objects, $\left\langle J_{\text {subs }}\right\rangle$, to the APS is negligible for $\ell \gtrsim 4$, so this is not discussed further.
} 

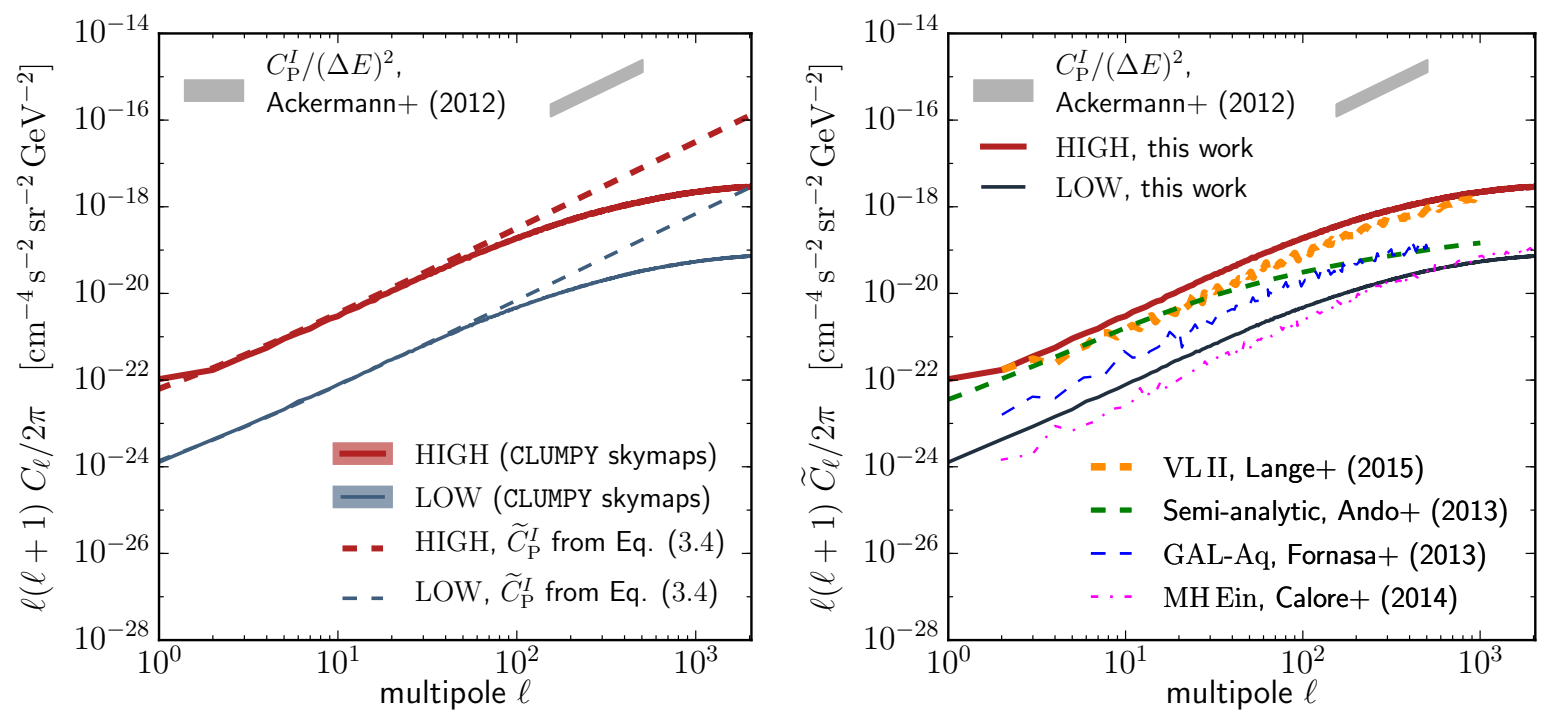

Figure 7: Left: Differential subhalo intensity APS at $4 \mathrm{GeV}$ and its variance on the full sky. The bands show the $68 \%$ CI around the median power at each $\ell$ for the models LOW (pale blue) and HIGH (red), obtained from 500 realisations. The dashed lines give the constant $C_{\ell}=\widetilde{C}_{P}^{I}$, according to Eq. (3.4). At high- $\ell$, the power spectra deviate from the point-source approximation due to the angular extension of the subhalos. The anisotropy level measured in the DGRB, in the multipole range $155 \leq \ell \leq 504$, from 22 months of observation with Fermi-LAT [143] ( $\sigma \sigma$ error band) is shown for comparison (see text for discussion). Right: Median values for the models LOW (dark grey) and HIGH (red), as on the right figure, compared to other works (dashed colours, see text for details). For the particle physics term, $m_{\chi}=200 \mathrm{GeV}, \chi \chi \rightarrow b \bar{b}$, and a thermal relic cross-section has been chosen.

$\mathcal{L}$ as defined in [86]. It is straightforward to show that

$$
C_{\mathrm{P}}^{I}=\frac{1}{4 \pi} \int_{F_{\min }}^{F_{\max }} F^{2} \frac{\mathrm{d} N}{\mathrm{~d} F} \mathrm{~d} F,
$$

where $N$ is the total number of subhalos, and $F$ their flux at the observer.

APS properties and model comparison. To facilitate the comparison to previous works [145-147], the APS below is given as differential intensity power at $E=4 \mathrm{GeV}$ for a $m_{\chi}=200 \mathrm{GeV}, \chi \chi \rightarrow b \bar{b}$ annihilation channel with thermal relic cross-section $\langle\sigma v\rangle=$ $3 \cdot 10^{-26} \mathrm{~cm}^{3} \mathrm{~s}^{-1}$ (same as in figure 5).

The left panel of figure 7 shows the median APS of the LOW and HIGH models and their $68 \%$ confidence intervals, CI, along with the median Poisson-like $C_{P}^{I}$ given in Eq. (3.4), calculated for each model and 500 simulations. Here, the flux $F_{\min }$ in Eq. (3.4) has been set to the flux from the faintest drawn object in each realisation. As shown in appendix C, further lowering $F_{\min } \rightarrow 0$ adds negligible contribution to the integral. The flux $F_{\max }$ in Eq. (3.4) is $F_{\max }=F^{\star}$, the flux from the brightest object in each skymap. The two approaches agree but at high- $\ell$ where the point-like approximation is not valid anymore. Additionally, the medians also differ at low- $\ell$ for the model HIGH, as the spatially isotropic distribution of the objects is violated here (see next paragraph). A main benefit from our approach is to properly propagate the $\ell$-dependent uncertainties on the APS (see left panel), which is 
also not limited to a single simulation and its limited mass resolution; the cosmic-variance uncertainty is of almost one order of magnitude.

The right panel in figure 7 shows the median $C_{\ell}$ for the models LOW (black) and HIGH (red), which encompass the results based on either the Via Lactea II [145] or Aquarius simulations [146]. The APS from Galactic subhalos obtained by [148] (green dashed line in figure 7) is also based on the Aquarius simulations. ${ }^{11}$ However, they semi-analytically computed the spectrum from a generalized version of Eq. (3.3), additionally taking into account a suppression factor $|\tilde{u}(\ell)|^{2}$ due to the spatial extension of the subhalos. In the g15784 simulation by [147] (magenta dot-dashed line in figure 7), only subhalos with masses $>10^{8.6} \mathrm{M}_{\odot}$ are resolved, which explains the lower overall power. Beyond the validity check, the subhalo APS is an interesting tool for the observational search strategy. Figure 7 shows that at low multipoles, a small dipole excess $(\ell=1)$ is visible for the model HIGH (and none for the model LOW). Therefore, we checked if a spatial bias exists for the location of the brightest clumps. In figure 9 , we show the probability distribution to find the brightest object at angular distance $\theta$ from the direction of the GC for LOW (pale blue) and HIGH (red). For model LOW, only a marginal spatial preference exists to find the brightest halo in the direction of the GC. This is not the case for model HIGH, where the direction of the GC is clearly preferred. ${ }^{12}$ Cross-checking with model VAR6, we find this change to be mostly due to the distance-dependent concentration of model HIGH: the closer subhalos are to the $\mathrm{GC}$, the more concentrated, hence brighter, they become. We come back later on the fact that the brightest objects are close-by with $D_{\text {obs }} \approx R_{\odot} \approx \mathcal{O}(10 \mathrm{kpc})$ from both the observer and the GC.

APS and DM sensitivity. The APS can be compared to existing data on the anisotropy in the diffuse $\gamma$-ray background (DGRB, see, e.g., [47]). After 22 months of data-taking, Fermi-LAT reported a significant excess $(>3 \sigma)$ of $\gamma$-ray anisotropy over the photon noise background in the diffuse emission at latitudes $|b|>30^{\circ}$, in each of four different energy intervals between $1 \mathrm{GeV} \leq E \leq 50 \mathrm{GeV}$, and in the multipole range $155 \leq \ell \leq 504$ [143]. We take their DATA:CLEANED result, given as appropriately rescaled full-sky equivalent power, in differential form at $4 \mathrm{GeV}$. It is shown in figure 7 as a grey-shaded segment vertically extending over the $\pm 1 \sigma$ uncertainty range. Accounting for the dominant contribution of distant unresolved blazars [149] and misaligned active galactic nuclei [150] would lower the differential intensity angular power by a factor of a few, leading to a residual anisotropy that could be attributed to DM. For instance, several authors have used the measurement from [143] and the blazar contribution from [149, 150] to derive upper limits on the relic cross-section $\langle\sigma v\rangle[145,148,151,152]$.

A full analysis of the constraints set by the APS data is beyond the scope of this analysis. Nevertheless, thanks to our self-consistent derivation of the APS and source count distribution of Galactic DM subhalos, we may comment on the sensitivity of the former, and the relative merit of both approaches. Using the result of [143], based on 22 months

\footnotetext{
${ }^{11}$ The curve for the Galactic APS from [148] has been rescaled for comparison to the other works. We adopted $\mathrm{d} \Phi^{\mathrm{PP}} / \mathrm{d} E\left(4 \mathrm{GeV}, m_{\chi}=200 \mathrm{GeV}, \chi \chi \rightarrow b \bar{b}\right)=5.63 \cdot 10^{-32} \mathrm{GeV}^{-3} \mathrm{~cm}^{3} \mathrm{~s}^{-1}$, compared to $\Phi^{\mathrm{PP}}\left(E_{\min }=\right.$ $\left.5 \mathrm{GeV}, E_{\max }=10 \mathrm{GeV}, m_{\chi}=100 \mathrm{GeV}, \chi \chi \rightarrow b \bar{b}\right)=2.07 \cdot 10^{-31} \mathrm{GeV}^{-2} \mathrm{~cm}^{3} \mathrm{~s}^{-1}$, thus rescaling by a factor $(0.56 / 2.07)^{2} \mathrm{GeV}^{-2}$. However note that the spectral shape for the $\chi \chi \rightarrow b \bar{b}$ channel assumed by [148] slightly differs from ours.

${ }^{12}$ Note that we only consider the subhalo emission, not the DM emission from the smooth Galactic halo, which is highly peaked towards the GC. Therefore, this discussion does not apply for the brightness in terms of signal to background ratio.
} 


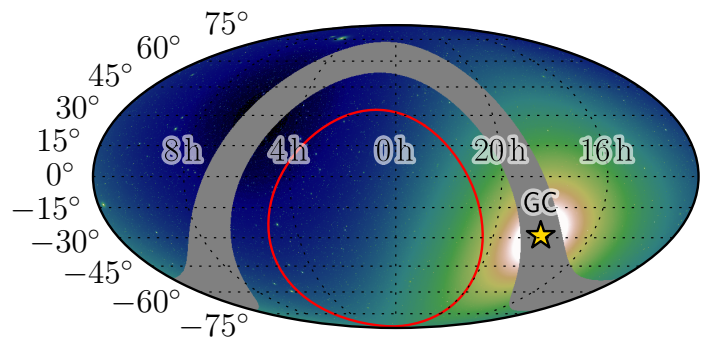

Figure 8: Masked skymap (equatorial coordinates) for our Fermi-LAT setup (grey band around the Galactic plane) and the assumed CTA survey field in this study (within the red line). The colormap in the back is the same as in figure 5 (model LOW, total emission).

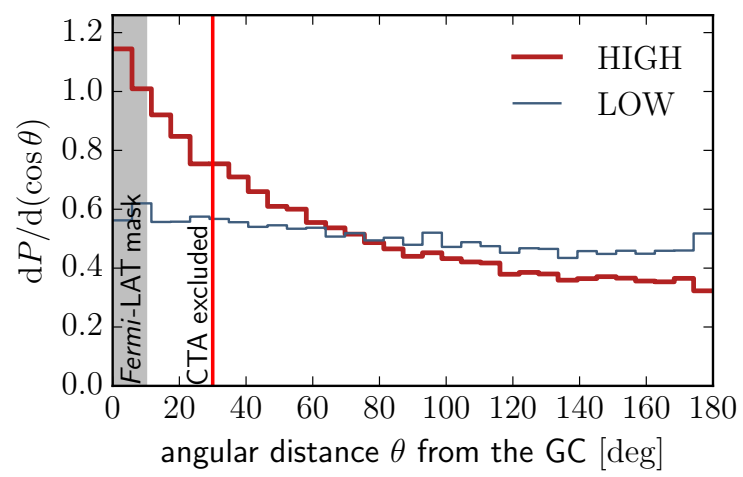

Figure 9: Probability to find the brightest subhalo at the angular distance $\theta$ from the GC. The vertical lines indicate the sky cuts of each instrument (shown in figure 8).

of Fermi-LAT data with 1FGL point sources removed, Ando and Komatsu [148] typically find upper limits of $\langle\sigma v\rangle \sim 10^{-24} \mathrm{~cm}^{3} \mathrm{~s}^{-1}$ for $m_{\chi}=200 \mathrm{GeV}$ and $b \bar{b}$ (see their figure 16). An order-of-magnitude calculation performed from figure 7 (we recall that $C_{\ell} \propto\langle\sigma v\rangle^{2}$ ) for models LOW and HIGH encompasses this value, with model HIGH yielding the lowest limit. This work also shows that APS-derived limits are affected by a cosmic-variance uncertainty of almost one order of magnitude. Alternatively, looking for dark clumps in the 3FGL catalogue (48 months of data), Schoonenberg et al. [81] find $\langle\sigma v\rangle \sim 10^{-25} \mathrm{~cm}^{3} \mathrm{~s}^{-1}$ (see their figure 7). Their underlying subhalo model is in line with our model HIGH prediction (see figure 10), and also suffers from a similar variance uncertainty. From these crude considerations, the APS approach appears to be somewhat less favourable than dark clump searches. Considering many effects such as data homogeneity, masking effects, etc., the comprehensive study of [145] finds similar results. In the context of a large-sky survey with the CTA instrument, we present the CTA sensitivity to dark clumps in section 5. Comparing those results to anisotropy searches with CTA, as suggested by [153], is left for future work.

\section{Revisiting dark halo searches with Fermi-LAT}

The subhalo detection prospects for Fermi-LAT have been investigated several times [6367, 79-81]. This short section aims at comparing our work to the recently published results of $[80,81]$ and at commenting on some differences compared to a CTA-like survey (presented in section 5). To do so, we now move from the full sky approach of the previous section to a setup tailored to the Fermi-LAT experiment. Following [81], we (i) investigate the subhalo population outside the Galactic plane at $|b|>10^{\circ}$, and we adopt the same region of interest, as shown in figure 8; (ii) limit the $J$-factor integration angle to $\theta_{\text {int }}=0.8^{\circ}$, as done by [81], describing Fermi-LAT's $68 \%$ containment radius at $1 \mathrm{GeV}$.

Figure 10 shows the cumulative source count distributions for this setup and the subhalo models LOW (pale blue) and HIGH (red). The lower $x$-axis presents the subhalo source count distribution in terms of the particle-physics independent $J$-factor, while the upper $x$-axis gives the corresponding integrated energy flux distribution above $1 \mathrm{GeV}$ (for $m_{\chi}=100 \mathrm{GeV}$, thermal annihilation cross-section, and pure annihilation into bottom quarks). This allows 


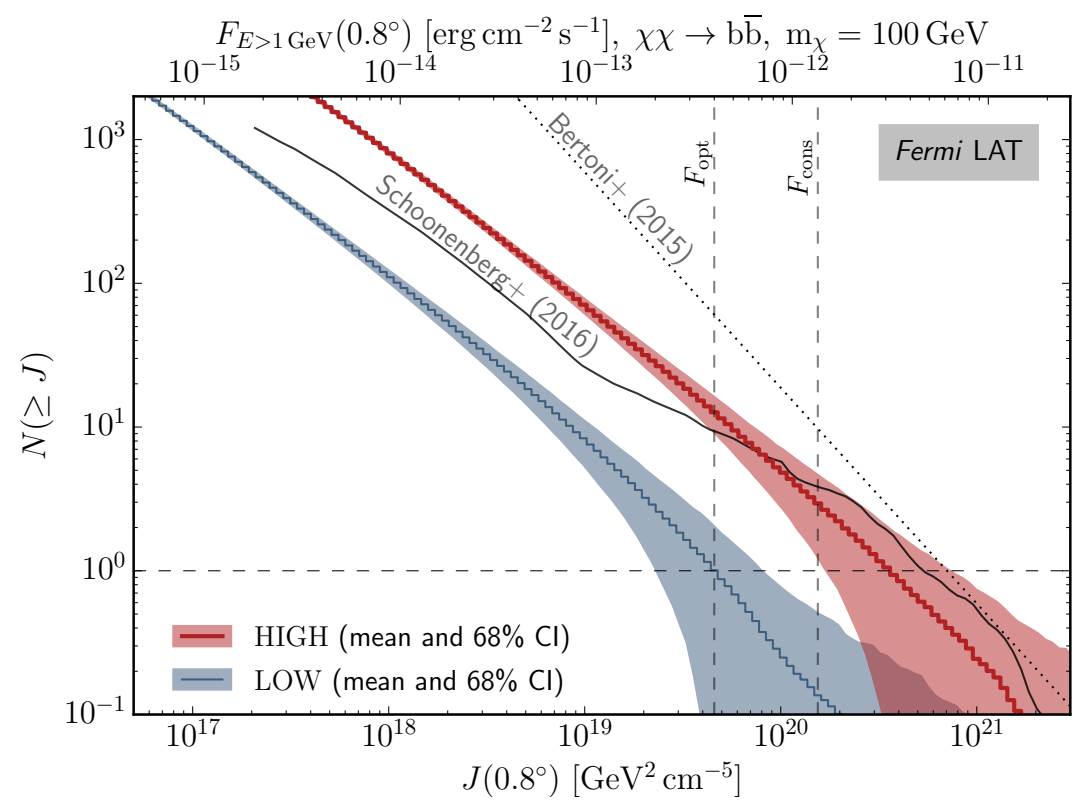

Figure 10: Cumulative source count distribution of DM subhalos for the Fermi-LAT setup. The coloured bands denote the $1 \sigma$ standard deviation around the mean $\bar{N}$ from the 500 simulations. The lower $x$-axis gives $J$-factors and the upper $x$-axis the corresponding flux for a given particle physics model, using $\langle\sigma v\rangle=3 \cdot 10^{-26} \mathrm{~cm}^{3} \mathrm{~s}^{-1}$. The Bertoni et al. [80] (dotted line) and Schoonenberg et al. [81] results (solid line) are also displayed. The vertical dashed lines show the conservative and optimistic detection thresholds chosen by [81]. Taking into account an up-to-date LAT sensitivity (see text), these thresholds would move by an approximate factor 2 to the left.

us to directly compare our modelling to the findings of Schoonenberg et al. [81] (black solid line $)^{13}$ and, with some limitations, Bertoni et al. [80] (black dotted line). Ref. [80] considers only subhalos at $|b|>20^{\circ}$, while we use $|b|>10^{\circ}$, meaning that, compared to our setup, the dotted line should be even higher than that shown in figure $10 .{ }^{14}$ For low $J$ values, HIGH and LOW nicely encompass the Schoonenberg et al. results. For the largest $J$-factors, the HIGH model is consistent with [81] within uncertainties, ${ }^{15}$ and in some tension with [80].

The authors of [81] estimate the number of detectable subhalos for a specific DM particle physics model from the Fermi-LAT detection threshold in the 3FGL, for sources with a similar, relatively hard spectral shape as expected from DM annihilations. In particular considering the $\chi \chi \rightarrow b \bar{b}$ channel, they assume a conservative detection threshold $F_{\text {cons }, \mathrm{b} \overline{\mathrm{b}}}=1.35 \cdot 10^{-12} \mathrm{erg} \mathrm{cm}^{-2} \mathrm{~s}^{-1}$, and an optimistic detection threshold $F_{\text {opt, } \overline{\mathrm{b}}}=$ $4.0 \cdot 10^{-13} \mathrm{erg} \mathrm{cm}^{-2} \mathrm{~s}^{-1}$ (both fluxes integrated above $1 \mathrm{GeV}$ ). For $F_{\mathrm{opt}}$, bु and the particle physics model chosen in figure 10, model HIGH predicts $13 \pm 4$ detectable subhalos to be present in the 3FGL, while still $1 \pm 1$ halo could be found for the conservative model LOW;

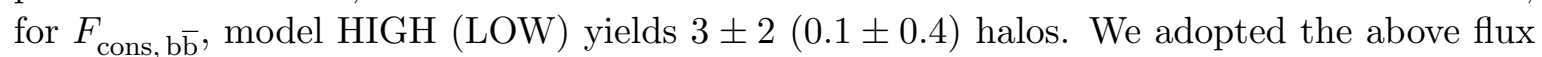
thresholds and quote the number of detectable clumps based on the 3FGL to ease comparison

\footnotetext{
${ }^{13}$ We added their distributions of point-like and extended halos which they discuss separately. Note that they use a simplified approach to calculate the $J$-factors. Performing the full line-of-sight integration in CLUMPY, we do not find a strict difference between point-like and extended objects.

${ }^{14}$ The integration angle used in [80] is not specified and the comparison is only valid provided that their calculation of the DM spectra is consistent with [102].

${ }^{15}$ Ref. [81] finds a similar sample variance of $N(68 \% \mathrm{CI})$, which is not shown in figure 10.
} 
with the results found by [81]. However, after eight years of operation, and with the improved Pass8 event reconstruction [140], the LAT sensitivity to faint sources has significantly improved since the 3FGL release. For the background-dominated regime between $1 \mathrm{GeV}$ and $10 \mathrm{GeV}$, the double exposure yields a factor $\sqrt{2}$ in improved sensitivity, and the Pass 8 reconstruction additionally improves the sensitivity by about $30 \%$ compared to the Pass 7 analysis chain. ${ }^{16}$ With this, we approximate the available flux thresholds above $1 \mathrm{GeV}$ after eight years observations with Pass8 reconstruction to be $F_{8 \text { years }} \approx 0.5 F_{3 \mathrm{FGL}}$. Correspondingly, the number detectable clumps increases by factor $\sim 0.5^{1-\alpha} \sim 2$, with $1-\alpha \approx-1$ the slope of the source count distribution in figure 10. Therefore, assuming $\langle\sigma v\rangle \approx 3 \cdot 10^{-26} \mathrm{~cm}^{3} \mathrm{~s}^{-1}$ and for $m_{\chi} \lesssim 100 \mathrm{GeV}, \mathrm{DM}$ subhalos might already have been detected by Fermi-LAT, even under conservative assumptions about the subhalo model and the detection threshold. Conversely, we remark that an exclusion of the DM hypothesis for most of the unidentified 3FGL objects is consistent with Fermi-LAT's finding from stacked dSph galaxy observations, $\langle\sigma v\rangle<3 \cdot 10^{-26} \mathrm{~cm}^{3} \mathrm{~s}^{-1}$ for $m_{\chi}<100 \mathrm{GeV}$ [30], and these limits are now more stringent after four more years data taking.

We remark that the Fermi-LAT angular resolution strongly improves at energies above $1 \mathrm{GeV}$, reaching a $68 \%$ containment radius of $0.2^{\circ}$ at $\sim 10 \mathrm{GeV}$, and $0.1^{\circ}$ above $\sim 100 \mathrm{GeV}[15$, 140]. Therefore, we stress that the choice of $\theta_{\text {int }}=0.8^{\circ}$ in this paragraph is not a description of the LAT performance, but serves for comparison of the source count distribution from [81].

For the plausibility of dark subhalo searches, it is of interest to investigate the physical properties (mass, distance, angular extension) of the brightest subhalo. The median properties of the brightest object within the masked Fermi-LAT sky and for the model HIGH are presented in table 3 . The properties of the brightest subhalo may depend on the angular resolution of the instrument and we find that the object with the largest overall $J$-factor, $J\left(\theta_{\text {vir }}\right)$, is not necessarily the same as the object with the largest $J$-factor within some $\theta_{\text {int }}<\theta_{\text {vir }}$. This is accounted for in table 3 , and the brightest object is listed separately within the integration angles $\theta_{\text {vir }}, 0.8^{\circ}$ and $0.1^{\circ}$. However, this differentiation results into barely different objects within the resolutions of Fermi-LAT (in contrast to CTA, which is discussed later). For Fermi-LAT, the subhalo with the median largest $J$-factor is typically $8_{-6}^{+11} \mathrm{kpc}$ away, and has a mass between $\sim 10^{7} \mathrm{M}_{\odot}-10^{9} \mathrm{M}_{\odot}$.

We finally remark that in case of potential DM subhalo candidates, alternative origins for the signal (VHE blazars, milli-second pulsars) have to be ruled out. Various approaches for disentangling DM and astrophysical sources are presented in [75, 76, 81, 154]. Especially CTA will be a suitable instrument to perform dedicated follow-up observations of source candidates, and to resolve different origins by the temporal, spectral and spatial morphology of a candidate. Besides, CTA will be able to perform a large-area survey on its own, entering an energy regime beyond the accessibility of Fermi-LAT. In the following, we will now investigate whether a CTA survey can be used to search for DM subhalos.

\section{CTA sensitivity to dark clumps}

This section presents the CTA sensitivity to dark clumps (for the sake of readability, all the technical details are postponed to appendix E). We first introduce the salient features of CTA and CTA's extragalactic survey ( $§ 5.1$ ), present the instrument background ( $(5.2)$, the characteristics of the brightest clump for CTA $(\S 5.3)$, the likelihood and statistics used to draw

\footnotetext{
${ }^{16}$ http://www.slac.stanford.edu/exp/glast/groups/canda/lat_Performance.htm
} 


\begin{tabular}{|c|ccc|}
\hline $\begin{array}{c}\text { Energy } \\
\text { threshold }\end{array}$ & $\begin{array}{c}\text { Diffuse } \gamma \text {-ray rate } \\
{\left[\mathrm{Hz} \mathrm{deg}^{-2}\right]}\end{array}$ & $\begin{array}{c}\text { Background rate } \\
{\left[\mathrm{Hz} \mathrm{deg}^{-2}\right]}\end{array}$ & $\begin{array}{c}\gamma \text {-rays/background } \\
\text { ratio }\end{array}$ \\
\hline $30 \mathrm{GeV}$ & $2.8 \cdot 10^{-3}$ & 6.1 & $0.5 \cdot 10^{-3}$ \\
$100 \mathrm{GeV}$ & $1.1 \cdot 10^{-3}$ & 1.1 & $1.1 \cdot 10^{-3}$ \\
$300 \mathrm{GeV}$ & $3.0 \cdot 10^{-4}$ & 0.27 & $1.2 \cdot 10^{-3}$ \\
\hline
\end{tabular}

Table 2: Diffuse $\gamma$-ray and background rates after gamma-hadron separation cuts for the CTA on-axis performance, integrated over the energy above different energy thresholds.

our CIs ( $\S 5.4$ ), and then the ensuing sensitivity for CTA to these objects ( $§ 5.5)$. We underline that the knowledge of the probability distribution of the brightest clumps is mandatory to set robust constraints on DM detection. As discussed and shown in appendix B.2, this distribution has a long tail, so that the construction of credible intervals (CI) must rely on large samples to be correctly defined. For this reason, all the results presented in this section are based on $10^{4}$ CLUMPY runs of the models LOW and HIGH.

\subsection{Observation setup and nominal sensitivity}

CTA is the next-generation ground-based $\gamma$-ray observatory, using the technique of imaging atmospheric Cherenkov radiation ('Imaging Atmospheric Cerenkov Telescope', IACT). It will feature an unprecedented resolution in energy and angular separation for $\gamma$-rays in the range between $30 \mathrm{GeV} \lesssim E_{\gamma} \lesssim 200 \mathrm{TeV}$, and, over the whole energy range, an effective collection area of about an order of magnitude larger than current IACT [82]. Having a large field-ofview, CTA will be the first IACT to efficiently perform large-area surveys in VHE (very high energy, $\gtrsim 100 \mathrm{GeV}$ ) $\gamma$-rays. In particular, it plans to perform an extragalactic survey with an approximately uniform exposure over $25 \%$ of the sky [85]. This survey aims at an unbiased population study of extragalactic sources, primarily to search for 'dark particle accelerators' without any counterparts at other wavelengths. Analogously, it can be used for a search for Galactic DM annihilation in dark subhalos.

CTA will consist of two arrays, one in the northern and one in the southern hemisphere. We assume, for simplicity, that most of the survey will be performed by the southern array, in circular region around the Galactic south pole $\left(b<-30^{\circ}\right) .{ }^{17}$ This choice covers the projected $f_{\text {sky }}=25 \%$, as illustrated in figure 8 , and excludes the area close to the GC (see figure 9), where, for the model HIGH, bright subhalos are found with the highest probability; therefore, scanning lower Galactic latitudes or the combination with a Galactic plane survey could in principle increase the sensitivity to dark halos. Around 400 to 600 hours will be available for the extragalactic survey with CTA [85]. With an uniform distribution of this observing time over the whole survey area, a sensitivity to fluxes of about $2.5 \cdot 10^{-12} \mathrm{~cm}^{-2} \mathrm{~s}^{-1}$ to $1 \cdot 10^{-11} \mathrm{~cm}^{-2} \mathrm{~s}^{-1}$ above $100 \mathrm{GeV}$ (approximately corresponding to $0.5 \%-2 \%$ the flux of the Crab nebula [155]) can be reached for a Crab-nebula-like energy spectrum [85]. We discuss further the uniform observation strategy in the context of dark subhalos in appendix E.1.

\subsection{Diffuse $\gamma$-rays and residual background}

Earth-based $\gamma$-ray observatories suffer from a large residual background from cosmic rays. While sophisticated analysis techniques are able to efficiently discriminate the $\gamma$-ray signal

\footnotetext{
${ }^{17}$ Depending on the final location/latitude of CTA (southern site), a substantial part of the extragalactic survey area has to be covered by CTA North. Moreover, some of the area we selected may be unreachable for CTA (only observable at elevations $<40^{\circ}$ ), or the survey may reach smaller Galactic latitudes $|b| \lesssim 10^{\circ}$.
} 


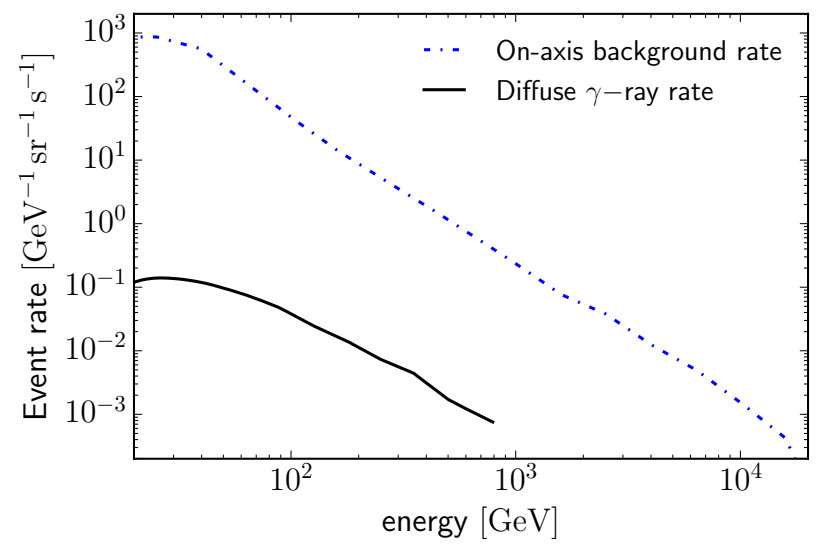

Figure 11: Diffuse $\gamma$-ray and background rates after gamma-hadron separation cuts for the CTA on-axis performance. The diffuse $\gamma$-rays (black solid line) are taken from [156] and comprise the intensity of all $\gamma$-rays above $|b|>20^{\circ}$, measured by Fermi-LAT up to $820 \mathrm{GeV}$, including the resolved sources. The background rate (blue dashed-dotted line) is given on-axis, optimised for 30 minutes of observation (as used throughout this paper).

from the hadronic background, it is almost impossible to separate it from cosmic electrons. In figure 11, we show the residual background rate estimation for CTA (blue dashed-dotted line), after applying background rejection cuts optimised for an observation over $30 \mathrm{~min}$, the typical observing time per field. This background rate is computed by MC simulations of the detector response to the cosmic-ray intensity, and represents the events passing the analysis cuts. In black, we show the event rate of the diffuse $\gamma$-rays, resulting from integrating the $\gamma$-ray intensity over the CTA on-axis effective area. We take the total $\gamma$-ray intensity above $|b|>20^{\circ}$, measured by Fermi-LAT up to $820 \mathrm{GeV}$ [156], to compare it with the CTA residual background. This intensity contains all $\gamma$-rays outside the Galactic plane, from resolved and unresolved sources. Thus, this spectrum gives a rather conservatively large value for the total diffuse $\gamma$-ray emission outside the Galactic plane. From figure 11 (differential rates) and table 2 (integrated rates), it can be seen that the residual background outweighs the diffuse $\gamma$-ray emission by a factor of 1000 . Therefore, we can safely ignore all diffuse $\gamma$-ray backgrounds on top of the residual cosmic-ray background, including those from Galactic and extragalactic DM.

\subsection{Characteristics of the brightest halos}

Similarly to the discussion in section 4 for Fermi-LAT, we investigate the source count distribution for a $J$-factor integration angle tailored to the angular resolution of the instrument, and display the result in figure 12 for models LOW and HIGH. We present the distributions for the integration angles $\theta_{\text {int }}=0.05^{\circ}$ (angular resolution of CTA at $\gtrsim 1 \mathrm{TeV}$ ), $\theta_{\text {int }}=0.1^{\circ}$ (angular resolution of CTA at $\lesssim 1 \mathrm{TeV}$ ), and the full emission, $\theta_{\text {int }}=\theta_{\text {vir }}$. Comparing figure 12 (CTA scenario) to figure 10 (Fermi-LAT scenario) shows that the CTA source count distributions shift to lower $J$-factors, due to a factor $\sim 3$ smaller survey field, and smaller integration angles. We also show in figure 12 the subhalo distribution assumed by [84] for a survey field characterised by $f_{\text {sky }}=25 \%$, based on the VL II subhalo catalogue. These authors consider an integration over the entire extent of the subhalos (i.e. $\theta_{\text {int }}=\theta_{\text {vir }}$ ), dismissing only highly extended subhalos, and their distribution is in fair agreement with our model HIGH. This is consistent with the fact that our model HIGH approximately matches 


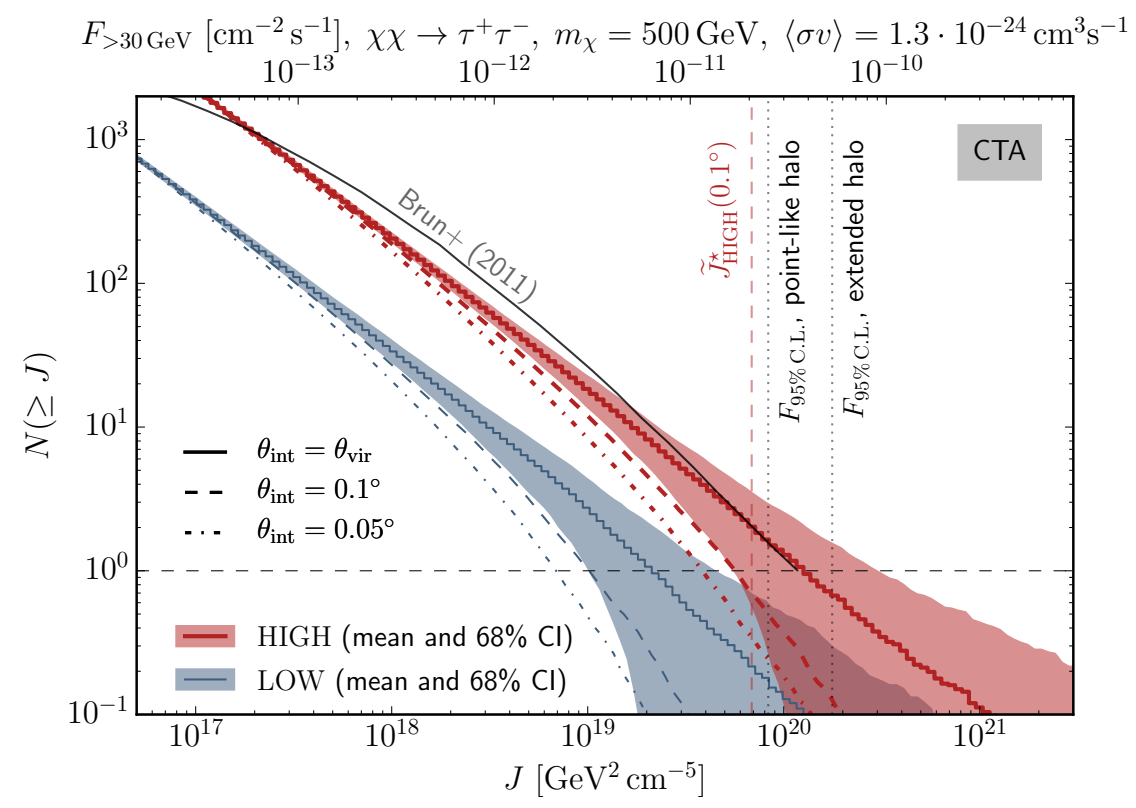

Figure 12: Cumulative source count distribution of DM subhalos for the CTA setup. The upper $x$-axis shows the flux level for the DM particle model to which CTA is most sensitive (adopting the subhalo model HIGH; see figure 13). The annihilation cross-section is chosen so that CTA would observe one subhalo above the flux sensitivity threshold corresponding to the chosen DM annihilation spectrum. Increasing (decreasing) the annihilation cross-section would result in a shift of the upper $x$-axis and the vertical dashed lines to the left (right). We also display the result of Brun et al. [84], who used $\theta_{\text {int }} \approx \theta_{\text {vir }}$.

the distribution derived from VL II also for the Fermi-LAT setup (see figure 10). However, we emphasize that assuming the whole subhalo flux, $J\left(\theta_{\text {vir }}\right)$, originating from a point source heavily overestimates the actual CTA performance. An exact treatment of the sensitivity must account for the energy dependent angular resolution and the extension of the source, and is done in the next subsections. In figure 12, we show the result from this rigorous treatment. The dashed lines show that for the considered DM particle, the sensitivity to the full emission from the brightest, extended halo (model HIGH) roughly corresponds to the sensitivity to a point source with the smaller flux within $0.1^{\circ}, J\left(0.1^{\circ}\right)$.

Table 3 lists the median properties of the brightest object for Fermi-LAT and CTA at various integration angles. The median $\widetilde{J}^{\star}\left(\theta_{\text {int }}\right)$ is obtained from $10^{4} \mathrm{MC}$ simulations (see also appendix B.2). In terms of halo properties, the population of brightest sources resembles the one for Fermi-LAT, and consists of close and rather massive halos; we remind the reader that we have identified the distance-dependence subhalo concentration as the main driver of this behaviour. The high angular resolution of CTA implies that changing the integration angle more drastically affects the brightest source properties than for FermiLAT: the smaller the integration angle, the lighter (and closer) the average brightest object becomes $\left(M_{\text {vir }} \gtrsim 10^{7} \mathrm{M}_{\odot}\right.$ for $\left.\theta_{\text {int }} \lesssim 0.05^{\circ}\right)$.

The subhalo source count distributions estimated so far include the most massive DM clumps, which would have formed stars and and host the dSph galaxies of the MW. Therefore, VHE $\gamma$-rays from DM annihilation in these objects will most likely be discovered by dedicated pointed VHE observations. Including dSph objects into the estimation of the CTA survey sensitivity to dark clumps might therefore bias the number of potentially detectable sources, even 


\begin{tabular}{|c|ccc|ccc|}
\hline \multirow{2}{*}{$\begin{array}{c}\text { Median properties of } \\
\text { brightest subhalo within }\end{array}$} & \multicolumn{3}{|c|}{ Fermi-LAT scenario $\left(f_{\text {sky }}=82.6 \%\right)$} & \multicolumn{3}{c|}{ CTA scenario } \\
$\left(\theta_{\text {int }}=0.1^{\circ}=25 \%\right)$ \\
\hline$\theta_{\text {int }}=0.8^{\circ}$ & $\theta_{\text {int }}=\theta_{\text {vir }}$ & $\theta_{\text {int }}=0.05^{\circ}$ & $\theta_{\text {int }}=0.1^{\circ}$ & $\theta_{\text {int }}=\theta_{\text {vir }}$ \\
\hline$\widetilde{D}_{\text {obs }}^{\star}[\mathrm{kpc}]$ & $7_{-5}^{+10}$ & $8_{-6}^{+11}$ & $8_{-6}^{+12}$ & $7_{-5}^{+10}$ & $8_{-6}^{+12}$ & $10_{-8}^{+16}$ \\
$\widetilde{R}^{\star}[\mathrm{kpc}]$ & $9_{-3}^{+9}$ & $10_{-3}^{+10}$ & $10_{-3}^{+11}$ & $10_{-2}^{+9}$ & $10_{-3}^{+10}$ & $12_{-4}^{+15}$ \\
$\log _{10}\left(\widetilde{m}_{\mathrm{vir}}^{\star} / \mathrm{M}_{\odot}\right)$ & $7.7_{-1.5}^{+1.3}$ & $8.1_{-1.6}^{+1.2}$ & $8.1_{-1.5}^{+1.3}$ & $7.4_{-1.4}^{+1.4}$ & $7.6_{-1.5}^{+1.4}$ & $8.0_{-1.6}^{+1.3}$ \\
$\widetilde{r}_{\mathrm{vir}}^{\star}[\mathrm{kpc}]$ & $6.7_{-4.6}^{+12}$ & $8.8_{-6.1}^{+14}$ & $9.2_{-6.3}^{+15}$ & $5.4_{-3.5}^{+9.5}$ & $5.9_{-4.0}^{+11}$ & $8.1_{-5.8}^{+14}$ \\
$\widetilde{r}_{\mathrm{s}}^{\star}[\mathrm{kpc}]$ & $0.13_{-0.10}^{+0.42}$ & $0.19_{-0.15}^{+0.55}$ & $0.21_{-0.17}^{+0.62}$ & $0.12_{-0.08}^{+0.36}$ & $0.14_{-0.10}^{+0.43}$ & $0.22_{-0.17}^{+0.69}$ \\
$\widetilde{c}_{\mathrm{vir}}^{\star}$ & $50_{-16}^{+23}$ & $44_{-15}^{+22}$ & $43_{-15}^{+22}$ & $45_{-14}^{+16}$ & $43_{-14}^{+17}$ & $37_{-13}^{+17}$ \\
$\widetilde{\theta}_{\mathrm{vir}}^{\star}[\mathrm{deg}]$ & $45_{-12}^{+16}$ & $48_{-12}^{+15}$ & $49_{-12}^{+14}$ & $37_{-11}^{+16}$ & $38_{-11}^{+15}$ & $39_{-10}^{+15}$ \\
$\widetilde{\theta}_{\mathrm{s}}^{\star}[\mathrm{deg}]$ & $1.2_{-0.6}^{+1.4}$ & $1.5_{-0.8}^{+1.6}$ & $1.6_{-0.8}^{+1.6}$ & $1.0_{-0.5}^{+1.1}$ & $1.1_{-0.5}^{+1.1}$ & $1.3_{-0.6}^{+1.1}$ \\
$\widetilde{\theta}_{\mathrm{h}}^{\star}[\mathrm{deg}]$ & $0.16_{-0.08}^{+0.20}$ & $0.20_{-0.10}^{+0.20}$ & $0.22_{-0.11}^{+0.22}$ & $0.13_{-0.05}^{+0.16}$ & $0.14_{-0.07}^{+0.14}$ & $0.18_{-0.08}^{+0.14}$ \\
$\log _{10}\left(\widetilde{J}^{\star} / \mathrm{GeV} \mathrm{cm}^{-5}\right)$ & $20.3_{-0.3}^{+0.4}$ & $20.7_{-0.3}^{+0.4}$ & $20.8_{-0.4}^{+0.5}$ & $19.7_{-0.3}^{+0.3}$ & $19.9_{-0.3}^{+0.4}$ & $20.3_{-0.4}^{+0.5}$ \\
\hline
\end{tabular}

Table 3: Median properties of the brightest subhalo for the survey setups tailored to the Fermi-LAT and CTA instruments, and for the subhalo model HIGH. The uncertainties denote the $68 \%$ CI around the median. For both instruments, the results for different angular resolutions are given. $D_{\text {obs }}$ is the distance from the observer, and $R$ the distance from the GC. $m_{\mathrm{vir}}$ is the subhalo mass. $r_{\mathrm{vir}}$ and $r_{\mathrm{s}}$ denote its virial and scale radius, $c_{\mathrm{vir}}=r_{\mathrm{vir}} / r_{\mathrm{s}}$, and $\theta_{\mathrm{vir}, \mathrm{s}}=\arctan \left(r_{\mathrm{vir}, \mathrm{s}} / D_{\mathrm{obs}}\right) . \theta_{\mathrm{h}}$ is the radius enclosing half of the total emission, $J\left(\theta_{\mathrm{h}}\right)=0.5 J\left(\theta_{\mathrm{vir}}\right)$. For reliable medians, the values are obtained from a sample of $10^{4}$ simulations.

more so when deep-sky and large-area optical surveys might discover additional faint dSph galaxies. To study the impact of this issue, we computed the subhalo abundance in the CTA scenario (25\% sky coverage), neglecting clumps heavier than $10^{6} \mathrm{M}_{\odot}$ or $10^{7} \mathrm{M}_{\odot}$ (Table 4 in the appendix shows that all the known dSph galaxies have a median mass larger than $10^{6} \mathrm{M}_{\odot}$, and most objects are likely to have masses larger than $\left.10^{7} \mathrm{M}_{\odot}\right)$. We find that when excluding all objects above $10^{7} \mathrm{M}_{\odot}$, the median $J$-factor within $\theta_{\text {int }}=0.05^{\circ}$ of the brightest subhalo is not affected, with $\log _{10}\left(\widetilde{J}^{\star}\left(0.05^{\circ}\right) / \mathrm{GeV}^{2} \mathrm{~cm}^{-5}\right)=19.7_{-0.2}^{+0.3}$. Looking over the full extent of the DM subhalo, we find $\log _{10}\left(\widetilde{J}^{\star}\left(\theta_{\text {vir }}\right) / \mathrm{GeV}^{2} \mathrm{~cm}^{-5}\right)=20.0_{-0.3}^{+0.4}$, i.e. a factor $\sim 2$ decrease compared to table 3 . This behaviour is understood as a lighter, but more concentrated halo is then selected as brightest object by the exclusion criterion. The situation changes when rejecting all objects above $10^{6} \mathrm{M}_{\odot}$, where we obtain $\log _{10}\left(\widetilde{J}^{\star}\left(0.05^{\circ}\right) / \mathrm{GeV}^{2} \mathrm{~cm}^{-5}\right)=19.5_{-0.2}^{+0.3}$ and $\log _{10}\left(\widetilde{J}^{\star}\left(\theta_{\text {vir }}\right) / \mathrm{GeV}^{2} \mathrm{~cm}^{-5}\right)=19.8_{-0.3}^{+0.4}$. The median brightest dark subhalo is then expected to be a factor 2 dimmer within the central $\theta_{\text {int }}=0.05^{\circ}$. The prospects of survey discoveries are therefore only marginally affected by the distinction between dark and bright DM halos. This point is not considered further in the remainder of this paper, where we use the values in table 3 (right) to characterize the brightest halo properties for a CTA extragalactic survey. 


\subsection{Likelihood ratio and test statistic (TS)}

We use the open-source CTA analysis software ctools $^{18}$, based on the gammalib library ${ }^{19}$ [157] to compute the CTA sensitivity to the median brightest dark subhalo. The ctools framework allows the use of a maximum-likelihood inference of hypotheses $\mathcal{M}$ from event data, considering all available spatial and spectral information from the data (see appendix E.3 for the likelihood $\mathscr{L}$ ). The cssens tool is used to simulate events and subsequently calculate the maximum log-likelihood ratio, with the likelihood ratio $\lambda$ given by

$$
\lambda=\frac{\max \mathscr{L}\left(\mathcal{M}_{\mathrm{bkg}}\left(\boldsymbol{\Theta}_{\mathrm{bkg}}\right) \mid \boldsymbol{X}\right)}{\max \mathscr{L}\left(\mathcal{M}_{\mathrm{sig}}\left(\boldsymbol{\Theta}_{\mathrm{sig}}\right)+\mathcal{M}_{\mathrm{bkg}}\left(\boldsymbol{\Theta}_{\mathrm{bkg}}\right) \mid \boldsymbol{X}\right)}=\frac{\mathscr{L}\left(\mathcal{M}_{\mathrm{bkg}}\left(\widehat{\widehat{\boldsymbol{\Theta}}}_{\mathrm{bkg}}\right) \mid \boldsymbol{X}\right)}{\mathscr{L}\left(\mathcal{M}_{\mathrm{sig}}\left(\widehat{\boldsymbol{\Theta}}_{\mathrm{sig}}\right)+\mathcal{M}_{\mathrm{bkg}}\left(\widehat{\boldsymbol{\Theta}}_{\mathrm{bkg}}\right) \mid \boldsymbol{X}\right)} .
$$

with $\boldsymbol{X}=\left(N_{\text {obs }}, E_{\text {obs }, 1 \ldots N_{\text {obs }}}, \vec{k}_{\text {obs }, 1 \ldots N_{\text {obs }}}\right)$ the mock data ${ }^{20}, \boldsymbol{\Theta}$ the adjustable parameters in the models maximising the likelihood, and $\widehat{\boldsymbol{\Theta}}$ the corresponding maximum likelihood estimators $\left(\widehat{\widehat{\Theta}}_{\text {bkg }}\right.$ under the constraint $\left.\mathcal{M}_{\text {sig }}=0\right)$.

For the background fit, we allow the normalisation of the rate to vary. The signal model $\mathcal{M}_{\text {sig }}$ for DM consists, according to Eq. (2.1), of the spatial part of our fixed $J$-factor map (see next subsection), and a spectral part depending on the particle mass $m_{\chi}$ and the annihilation cross-section $\langle\sigma v\rangle$. We scan $24 \mathrm{DM}$ particle masses in $50 \mathrm{GeV} \leq m_{\chi} \leq 100 \mathrm{TeV}$, computing for each $m_{\chi}$ the $\gamma$-ray spectrum $\mathrm{d} N_{\gamma}^{f} / \mathrm{d} E$ from [102]. For each spectrum, we set the flux level to be the only free parameter, such that $\boldsymbol{\Theta}_{\text {sig }}=\langle\sigma v\rangle$.

We use the logarithm of the likelihood ratio Eq. (5.1) as the test statistic TS to exclude the signal hypothesis $\mathcal{M}_{\mathrm{bkg}}+\mathcal{M}_{\text {sig }}$ (at the confidence level $1-p_{\text {pre }}$ ), namely

$$
\mathrm{TS}=-2 \log \lambda \text {. }
$$

The $\operatorname{TS}\left(p_{\text {pre }}\right)$ values were calculated directly from a set of MC simulations, and we refer the interested reader to appendix E.4 for the technical details and used TS values. More importantly, we cross-checked our analysis method by calculating the sensitivity to the Sculptor dSph galaxy, and found our results consistent with Carr et al.(2015) [90].

\subsection{Sensitivity to dark clumps and comparison to other targets}

To calculate the CTA sensitivity to the brightest subhalo in the survey field, we build a template of the median brightest object described in $\S 5.3$. We choose the $J$-factor profile to be that of the object found to be the brightest within $\theta_{\text {int }}=0.05^{\circ}$ (see table 3 ). We emphasize that the latter choice only determines the shape of the template halo, and the Likelihood-based sensitivity analysis is run over the full spatial extent of that halo. The instrument response, required in Eq. (5.1) and in the likelihood function Eq. (E.8), is taken from [83], using the publicly available results from the 'Production 2' simulations. ${ }^{21}$ The available instrument response data corresponds to on-axis observations of Crab-nebula-like point sources, which can be safely adopted for the highly cuspy DM template halo (with a half emission radius of $\theta_{\mathrm{h}}=0.13^{\circ}$, only slightly above the CTA angular resolution). We use the response tables with background rejection cuts optimised for a $30 \mathrm{~min}$. observation at a constant elevation of $70^{\circ}$ with CTA (southern site), ${ }^{22}$ assuming 4 large-size telescopes,

\footnotetext{
${ }^{18}$ http://cta.irap.omp.eu/ctools/

${ }^{19}$ http://cta.irap.omp.eu/gammalib/

${ }^{20} N, E$, and $\vec{k}$ are the number of photons, energy and direction respectively.

${ }^{21}$ http://portal.cta-observatory.org/Pages/CTA-Performance.aspx

${ }^{22}$ We cross-checked the analysis with the 'Production 2' rejection cuts optimized for a $5 \mathrm{~h}$ observation and obtained the same sensitivities.
} 

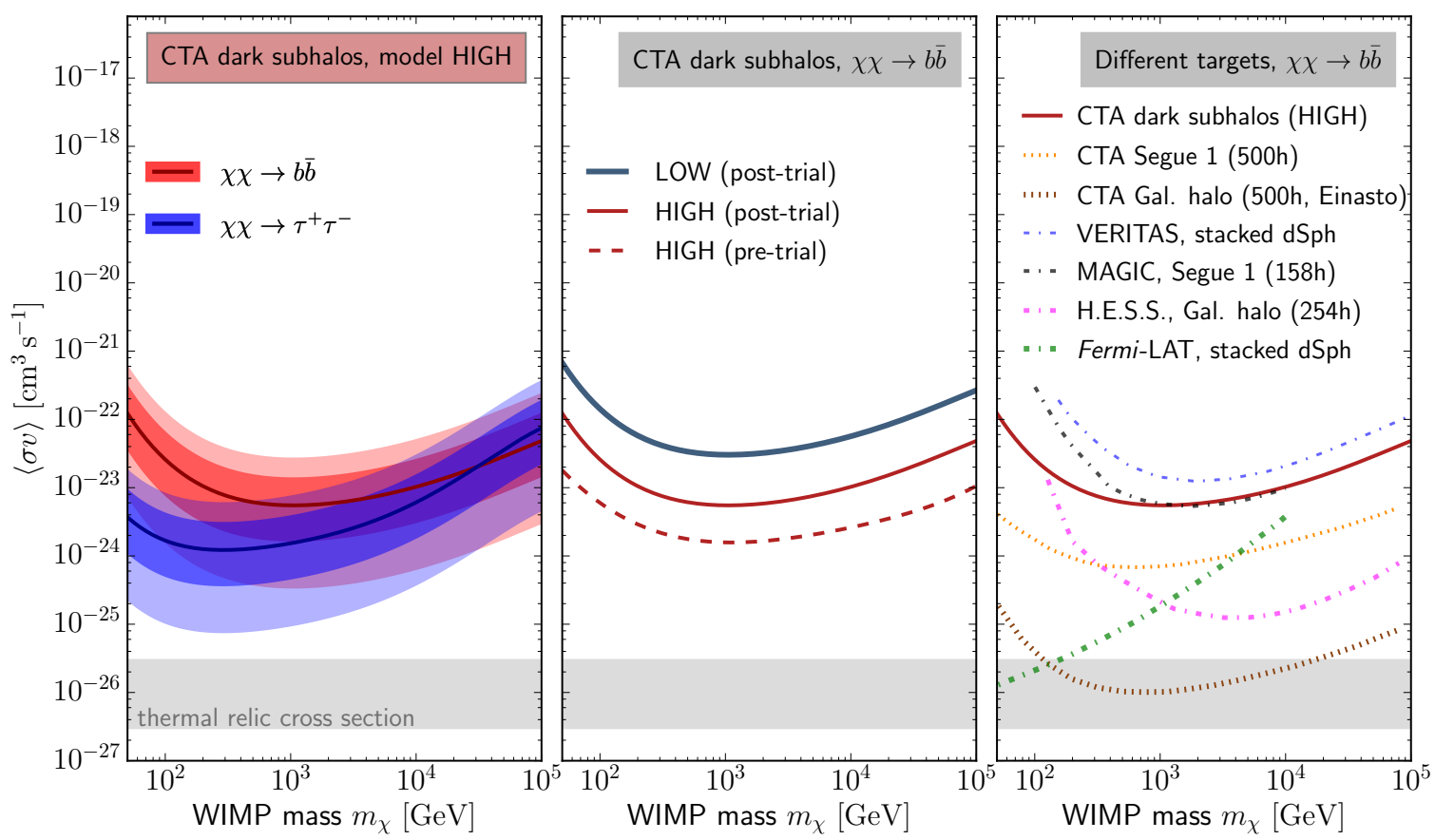

Figure 13: Sensitivity of the CTA extragalactic survey to find the brightest Galactic subhalo in the survey FOV. All sensitivities are given at the 95\% CL. Left: median (solid lines) and 68\% (95\%) $J$-factor (model HIGH) uncertainty around the median (coloured areas) for $b \bar{b}$ and $\tau^{+} \tau^{-}$annihilation channels. Centre: comparison of LOW, HIGH (pre- and post-trial). Right: Comparison of our analysis to the CTA sensitivity for other targets (Segue I and the GC from [90]) and to the limits from running experiments (VERITAS [35], MAGIC [37], H.E.S.S. [158], and Fermi-LAT [30]).

24 medium-size telescopes and 72 small-size telescopes. Different observation strategies have been proposed to raster the CTA survey field with overlapping observations to obtain a preferably homogeneous exposure [85]. We mimic the survey coverage by an on-axis observation of the template halo with a one hour exposure. With this choice, we calibrate our template observation setup to the optimistic benchmark performance projected for the CTA extragalactic survey, and obtain a similar sensitivity to a Crab-like point source as in [85]. ${ }^{23}$

In figure 13, we show the projected sensitivity of CTA to the WIMP DM annihilation cross-section by searching for Galactic dark subhalos in the planned extragalactic survey:

- In the left panel, we present the sensitivity for two benchmark annihilation channels with $100 \%$ branching ratio of $\chi \chi \rightarrow b \bar{b}$ and $\chi \chi \rightarrow \tau^{+} \tau^{-}$, respectively. The solid lines represent the sensitivity to the template halo with median $J$-factor. The shaded bands denote the $68 \%$ (95\%) statistical uncertainty around this median, originating from the $J$-factor variance. It can be seen that the sample-to-sample variation of the sensitivity scatters over almost one order of magnitude within the $68 \% \mathrm{CI}$, and two orders of magnitude within the $95 \%$ CI. Also, the sample variance has a long tail towards low

\footnotetext{
${ }^{23}$ We obtain project a survey sensitivity to fluxes $\gtrsim 4 \cdot 10^{-12} \mathrm{~cm}^{-2} \mathrm{~s}^{-1}$ above $100 \mathrm{GeV}$ (approximately $0.7 \%$ of the Crab nebula flux) for a point source with a Crab-nebula-like spectrum, requiring a test statistic of TS $=25$ and without applying a trials correction. This is in an optimistic agreement with [85]. For comparison, a point source with a spectrum $\chi \chi \rightarrow \tau^{+} \tau^{-}, m_{\chi}=500 \mathrm{GeV}$ (as discussed in figure 12), would be detected at $F_{\mathrm{TS}=25}=7.8 \cdot 10^{-12} \mathrm{~cm}^{-2} \mathrm{~s}^{-1}$ above $100 \mathrm{GeV}$.
} 
values of $\langle\sigma v\rangle$, such that despite a relative modest median sensitivity, the $95 \% \mathrm{CI}$ reaches relatively small annihilation cross-sections. The shape of the $J$-factor sample variance, which underlies the sensitivity variance, is discussed in detail in appendix B.

- In the central panel, we show the impact of the model uncertainty onto the sensitivity. The one order of magnitude difference in the predicted fluxes between the model LOW and HIGH translates into the equivalent difference in sensitivity. We also calculated the sensitivity at the $1-p_{\text {post }} \equiv 1-p_{\text {pre }}$ confidence level (see appendix E.2), neglecting the trials penalty (dashed line), that accounts for a putative improvement of the sensitivity by more than a factor of 5 .

- In the right panel, we put the sensitivity obtained from this study into broader context. A CTA survey search for dark subhalos provides a less powerful probe for DM annihilation than CTA pointed observations of the Galactic halo, and to a lesser extent, than the MW satellite galaxies. However, these other targets also suffer from systematic errors. Figure 13 also shows the limits from current experiments for comparison. It is visible that the GC provides the best limit at high energy, whereas the Fermi-LAT experiment already reaches the CTA parameter space below $\mathrm{TeV}$ energies.

Before concluding, we briefly comment on the results of Ref. [84], who previously discussed the CTA sensitivity to dark clumps. Based on the Via Lactea II simulation and a similar survey in area (1/4 of the sky, though towards the GC), they find a more favourable sensitivity than the one we obtain. For instance, in the $b \bar{b}$ channel, our calculation reaches a minimum (pre-trial) $\langle\sigma v\rangle \gtrsim 2 \times 10^{-24}$ to compare to $\langle\sigma v\rangle \gtrsim 4 \times 10^{-26}$ in [84]. Several reasons may be at the origin of this difference. First, our limit is based on the 95\% CL whereas theirs is based on $90 \%$ CL. With this choice, we find a factor of 2 improvement on the pre-trial sensitivity (the post-trial sensitivity is not affected). Second, Ref. [84] models the CTA instrument characteristics starting from the H.E.S.S. instrumental response, assuming a factor 10 larger effective area and a factor 2 better background rejection. However, the improvement of the CTA performance is energy-dependent, and according to the 'Production 2' simulations, the largest improvement in differential sensitivity compared to current instruments will be reached at energies above $\sim 1 \mathrm{TeV}$. Because CTA will be most sensitive to WIMP masses of $m_{\chi} \approx 1 \mathrm{TeV}$ (annihilation products below $1 \mathrm{TeV}$ ), the applied H.E.S.S. extrapolation most likely overestimated the CTA sensitivity to DM. Third, Ref. [84] assumes that the total subhalo $J$-factors, $J\left(\theta_{\text {vir }}\right)$, is enclosed in the instrumental resolution. This overestimates the flux of the brightest subhalo by another factor $\sim 2$. For these reasons, we are confident our analysis provides a more realistic estimate of the CTA sensitivity to dark subhalos.

\section{Summary and discussion}

In this paper, we have revisited the detectability of dark clumps for present and future $\gamma$-ray instruments. Using the CLUMPY code, we have simulated several distributions of the subhaloe in the Galaxy, in order to critically assess the range of potential number of detectable clumps and to identify the most important parameters of such prediction. For each model, several hundreds of skymaps have been generated to obtain the statistical properties (mass, distance, $J$-factor, etc.) for these configurations. The model dubbed HIGH (LOW) provides a realistic (conservative) benchmark model for the number of expected dark clumps. These benchmark models have been used to discuss the prospects of dark clumps detection for the Fermi-LAT 
and CTA instruments, and we compared our results and exclusion limits on annihilating DM to several previous calculations. We underline that we have carefully validated our analysis at each step of the calculation (validation and cross-checks for the distribution of $J$ and the APS from CLUMPY, and for the sensitivity calculations for CTA). Our findings are summarised below. First, for the substructure modelling, we find that:

- The concentration parametrisation is the main uncertainty in the determination of the number of halos with the largest $J$-factors. Indeed, the brightest detectable dark clumps are found to be close by, deep in the potential well of the Galaxy, where the impact of tidal stripping on the concentration and brightness is the most critical. As such, our LOW model serves as a baseline for a conservative estimate, in which no tidal stripping is accounted for (subhalos assumed to be as 'field' halos'). Our HIGH model is based on [67], which agrees well, in the regime that matters for detecting dark clumps, with the very recently estimated distance-dependent concentration parametrisation of Ref. [129]. This makes HIGH a likely realistic benchmark model, though further improvements and understanding of the stripping effect is necessary to strengthen this conclusion.

- The number of calibration subhalos $N_{\text {calib }}$ (between $10^{8}$ and $10^{10} \mathrm{M}_{\odot}$ ) and the boost from sub-subhalos in the external parts of the subhalos are the next impacting, though subdominant factors. First, moving from $N_{\text {calib }}=300$ in model HIGH to half this number in model VAR6, as hinted at by hydrodynamical simulations [105, 106], translates into a similar decrease for $J$ of the brightest object and the corresponding sensitivity. It is thus desirable to better constrain this number. Second, the boost factor is expected to be at most of a few for $10^{7}-10^{8} \mathrm{M}_{\odot}$ subhalos $[126,129]$. Compared to our conservative analysis in which this boost was discarded, this could slightly improve the prospects for Fermi-LAT, but probably not for CTA: for background-dominated instruments like CTA, the best sensitivity is achieved at the angular resolution (to decrease the background), for which the 'boosted' outskirts of the subhalos are not encompassed.

- The uncertainties in the other parameters (inner profile, index of the clump mass distribution, spatial distribution, width of the mass-concentration distribution) impact the overall level of the diffuse DM emission in the Galaxy, hence changes the contrast of the subhalos. However, it leaves mostly unchanged the number and signal strength of the brightest clumps.

A second aspect of our analysis, made possible by the reasonable running time of CLUMPY, is the determination of the statistical properties of the detectable clumps. We have shown that:

- The brightest clumps are typically located at $\sim 10-20 \mathrm{kpc}$ from the GC and from us, with concentration values $\sim 40-50$. Fermi-LAT and CTA probe slightly different populations, with larger and more massive subhalos for Fermi-LAT $\left(M \lesssim 10^{8} \mathrm{M}_{\odot}\right.$, $\theta_{\mathrm{h}} \lesssim 0.2^{\circ}$, and $\left.J \sim 3 \cdot 10^{20} \mathrm{GeV}^{2} \mathrm{~cm}^{-5}\right)$ than for CTA $\left(M \gtrsim 10^{7} \mathrm{M}_{\odot}, \theta_{\mathrm{h}} \gtrsim 0.1^{\circ}\right.$, and $\left.J \sim 10^{19} \mathrm{GeV}^{2} \mathrm{~cm}^{-5}\right)$. These halo masses and $J$-factors are close to the values obtained for dSph galaxies, and this raises the question whether the calculated sensitivity applies for truly dark halos or objects that could be discovered as nearby and very faint dSph galaxies in the future. However, we have shown that the calculated sensitivities are only mildly degraded (less than a factor 2) when rejecting masses above $M>10^{6} \mathrm{M}_{\odot}$ in a CTA large-sky survey scenario. In any case, CTA is sensitive to smaller mass ranges 
than Fermi-LAT. This difference is related to the angular resolution of the instruments $\left(\sim 0.05^{\circ}\right.$ for CTA compared to $\gtrsim 0.1^{\circ}$ for Fermi-LAT), illustrating that instruments with better angular resolution are sensitive to lower mass subhalos, which are more likely to be dark. CTA will therefore provide a complementary view to the observation of MW satellite galaxy observations.

- The properties of the brightest clumps derived from the statistical assessment underlie a large sample variance. The $J$-factor of the brightest clump varies by almost a factor 10 at $68 \% \mathrm{CI}(100$ at $95 \% \mathrm{CI})$. In particular, the distribution has a long tail towards large $J$-values. On the one hand, this means that a dark DM subhalo might be detected even for a relatively small annihilation cross-section. On the other hand, the large sample variance introduces a large systematic uncertainty for the limits derived from non-observation of DM candidates in the surveys.

- Tidal effects of subhalos, modelled here via the distance-dependent concentration, leads to an anisotropy in the distribution of the directions of the brightest clumps, as seen in the small dipole power excess (compared to models using field halos for the concentration leading to an isotropic distribution). The impact of this effect on detectability and sensitivity is not straightforward to assess: (i) the contrast of the dark clumps w.r.t. the DM diffuse emission decreases towards the GC, which should mostly affect the detectability when pointing towards $\theta \lesssim 10^{\circ}$; (ii) however, this should be balanced with the existence of a preferential direction to search for these dark halos (4 times more likely to lie towards the GC direction than towards the anticentre), for which the analysis could be optimised. In any case, the number of bright dark halos searched for in an analysis is small (by definition) and variance dominated at low $-\ell$. A dedicated study is necessary to assess the potential benefit of an optimised search.

Finally, a last aspect of our analysis deals with the sensitivity of CTA, and to some extent of Fermi-LAT, to dark clumps, compared to previous calculations and other targets:

- For an observation setup such as that of Fermi-LAT, a comparison of the expected number of clumps to previous calculations confirms that Ref. [80] obtain a subhalo abundance slightly larger than our model HIGH. Our results are more in line with those of Ref. [81], which discusses possible explanations for the difference to the results by [80]. However, compared to [81], we do not find two different populations and behaviours 'point-like' vs 'extended' source in our analysis (we perform the full integration along the line of sight, whereas [81] do not). As a result the shape of the number of expected dark clumps as a function of $J$ is not the same. The imprint of the population of DM galactic subhalos also shows on the APS, and our models LOW and HIGH encompass previously published studies.

- For a CTA large-sky survey scenario, we have based our sensitivity calculation on the projected extragalactic survey [85]. We provide both a pre-trial and post-trial sensitivity, as scanning a quarter of the sky results in a million independent trials that must be accounted for in setting the limits. For pure annihilations into $b \bar{b}$, we find the best post-trial median sensitivity reach of $\langle\sigma v\rangle \sim 6 \times 10^{-24} \mathrm{~cm}^{3} \mathrm{~s}^{-1}$ at $m_{\chi} \sim 1 \mathrm{TeV}$, and for the $\tau^{+} \tau^{-}$annihilation channel, the best sensitivity of $\langle\sigma v\rangle \sim 1 \times 10^{-24} \mathrm{~cm}^{3} \mathrm{~s}^{-1}$ at $m_{\chi} \sim 500 \mathrm{GeV}$. The $68 \%(95 \%)$ CI changes these values by a factor $\sim 5(\sim 10)$. 
In any case, taking the post-trial limit into account worsens the sensitivity by a factor $\sim 5$.

- The sensitivity reach of CTA (in model HIGH) for dark clumps in the extragalactic survey is quite competitive with dedicated dSph galaxies observations. Looking at the sensitivity based on the most-likely values favours the $500 \mathrm{hrs}$ single bright dSph observation over that of the extragalactic survey (factor $\sim 3$ ), but we recall that both suffer from large uncertainties. In particular, it has been shown that Segue 1's J-factor (used in our comparison plots) could be significantly lower than previously estimated $[98,141]$. In any case, the best target remains the GC region.

To conclude, we have shown that a CTA dark halo search provides a complementary view on a different population of subhalos compared to observing dSph galaxies. The planned CTA astronomical surveys will therefore nicely complement the dedicated DM searches programme (a similar analysis as done here could be performed for decaying dark matter). Given the unprecedented angular resolution of CTA, the search for small-scale anisotropies in its data, e.g., as proposed by [153], may provide another complementary constraint on $\gamma$-ray emission from Galactic dark clumps to that we have presented in this article.

\section{Acknowledgments}

This article has gone through internal review by the CTA Consortium, and we thank Hannes Zechlin and Josep Martí for their careful reading and suggestions that helped to improve the manuscript. We also thank the anonymous referee for useful suggestions that have improved the quality of the paper. We warmly thank V. Bonnivard for helping out with the Jeans analysis of the recently discovered $\mathrm{dSph}$ galaxies, and L. Gérard for valuable help with the CTA analysis. We also thank I. Sadeh for useful discussions and comments to the manuscript, and R. Kieokaew for performing useful studies preceding this work. This work has been supported by the Research Training Group 1504, "Mass, Spectrum, Symmetry", of the German Research Foundation (DFG), by the "Investissements d'avenir, Labex ENIGMASS", and by the French ANR, Project DMAstro-LHC, ANR-12-BS05-0006. Some of the results in this article have been derived using the HEALPix package [142].

\section{A Halo overdensity definition $\Delta$ and cosmology}

At a given redshift $z$, the enclosed mass $M_{\Delta}$ in a dark matter halo is generically defined as the mass contained within a radius $R_{\Delta}$ inside of which the mean interior density is $\Delta$ times the critical density $\rho_{c}$ :

$$
M_{\Delta}=\frac{4 \pi}{3} R_{\Delta}^{3} \times \Delta \times \rho_{c} .
$$

The spherical top-hat collapse model provides the virial overdensity $\Delta=\Delta_{\text {vir }}[159]$ which, for the family of flat cosmologies, $\Omega_{m}+\Omega_{\Lambda}=1$ (i.e., $\Omega_{K}=0$ ), can be approximated by $\Delta_{\text {vir }} \simeq\left(18 \pi^{2}+82 x-39 x^{2}\right) / \Omega_{m}(z)$, with $x=\Omega_{m}(z)-1, \Omega_{m}(z)=\Omega_{m}(0)(1+z)^{3} / E^{2}(z)$, and $E^{2}(z)=\Omega_{m}(0)(1+z)^{3}+\Omega_{\Lambda}[160]$. In this paper we use $\Omega_{m}(0)=0.308$ and $\Omega_{\Lambda}=0.692$ [161], leading to $\Delta_{\text {vir }} \simeq 332$.

In principle, the virial radius $R_{\text {vir }}$ can be used as a criterion to identify bound objects. In the spherical collapse model, this radius can be interpreted as a threshold separating a region within which the material is virialized and an external region where mass is still 
collapsing onto the object. Because the details of the collapse and virialization are not-well understood, several choices have been made in the literature to identify halos in simulations, such as $\Delta=\Delta_{\text {vir }}, 200,500, \ldots$ (see, e.g., [162]). We refer the reader to [163] for a study on whether there exists a more 'universal' choice.

CLUMPY works with $\Delta=\Delta_{\text {vir }}$, and it uses conversion formulae from [164] to convert '200' to 'vir' quantities, whenever necessary. This choice can in principle impact the estimation of the halo and subhalo mass shown in several plots of this study. However, in CLUMPY, the mass is mostly a proxy to calculate structural parameters of subhalos, via the $c_{\Delta}-M_{\Delta}$ relationship: the exact definition for $\Delta$ does not matter as long as the calculation and conversions are done consistently - the uncertainties on the signal are dominated by our ignorance on the dark matter subhalo population, not by the conversion factors. Then, for comparisons to the mass or $J$-factor of real data, as discussed in appendix $\mathrm{D}$, the uncertainties are larger than the difference one would obtain by using different choices for $\Delta$. Finally, we could also ask how sensitive is the $c_{\Delta}-M_{\Delta}$ relationship to cosmological parameters. For instance, in $\S 2.3$, the results based on the Via Lactea II [66], Aquarius [58], and ELVIS [165] simulations all start with different cosmologies (from WMAP 3 to WMAP 7 [166]). However, [129] finds a very weak dependence of $c_{\Delta}-M_{\Delta}$ on the cosmological parameters, within their statistical dispersion. We refer the reader to [167] for a pedagogical introduction to the link between various choices of $\Delta$, the $c_{\Delta}-M_{\Delta}$ relationship, and the cosmological parameters.

\section{B Mean and variance from analytical approximations}

Analytical formulae are always useful to cross-check numerical calculations and to better identify the underlying important parameters. Under the approximation of power-law source count distributions, we focus in this appendix on (i) the mean and median of the number of dark clumps above a given $J$-factor, which is ultimately related to the sensitivity of an instrument to dark clumps and on (ii) the mean and variance of the angular power spectrum of the $\gamma$-ray signal. The analytical results are compared to the direct calculations from our simulations in several places in the paper.

\section{B.1 Power-law source count distribution}

As seen in figure 3 , the mean number of halos $\bar{N}(>J)$ whose annihilation factor is larger than $J$ can be approximated by a power-law distribution over a large range of $J$-factors,

$$
\bar{N}(>J) \equiv\left\langle\int_{J}^{\infty} \frac{\mathrm{d} N}{\mathrm{~d} J^{\prime}} \mathrm{d} J^{\prime}\right\rangle \approx\left(\frac{J}{J_{\lim }}\right)^{1-\alpha},
$$

where $J_{\lim }$ is defined by $\bar{N}\left(>J_{\lim }\right)=1$, and provided that $\alpha>1 .^{24}$

\section{B.2 Mean and median of the brightest DM halo}

We define the probability $P_{\geq 1}$ to obtain at least one object brighter than a given flux $J$ as

$$
P_{\geq 1}(J)=\sum_{N=1}^{\infty} p[N(>J) \mid \bar{N}(>J)],
$$

\footnotetext{
${ }^{24}$ Eq. (B.1) can be easily generalized including an exponential cut-off at $J_{\mathrm{c}}, \quad \bar{N}(>\quad J)=$ $\left(J / J_{\lim }\right)^{1-\alpha} \exp \left[-\left(J-J_{\lim }\right) / J_{\mathrm{c}}\right]$, obtaining the form of a Schechter luminosity function [168].
} 
with $p(N \mid \bar{N})$ the probability density to obtain exactly $N$ objects brighter than $J$ for an expectation value of $\bar{N}$. If $p(N \mid \bar{N})$ follows a Poisson distribution, then the cumulative density function $P_{\geq 1}$ is given by

$$
P_{\geq 1}(J)=1-\exp [-\bar{N}(>J)]
$$

For example, Eq. (B.3) implies that one obtains at least one subhalo brighter than $J_{\text {lim }}$ with a chance of $1-e^{-1}=63 \%$. For $\bar{N}(>J)$ a power-law distribution, Eq. (B.1), plugged into Eq. (B.3), gives the probability density function

$$
\frac{\mathrm{d} P \geq 1}{\mathrm{~d} J}(J)=\frac{\alpha-1}{J_{\lim }} \exp \left[-\left(\frac{J}{J_{\lim }}\right)^{1-\alpha}\right]\left(\frac{J}{J_{\lim }}\right)^{-\alpha} .
$$

The expectation value $\bar{J}_{\geq 1}$ is calculated from Eq. (B.4) and the median $\widetilde{J}_{\geq 1}$ from Eq. (B.3). If the subhalo distribution can be approximated by a power-law distribution, Eq. (B.1), then $\bar{J}_{\geq 1}$ and $\widetilde{J}_{\geq 1}$ are suitable approximations for the mean and median of the brightest halo:

$$
\begin{aligned}
& \bar{J}^{\star} \approx \bar{J}_{\geq 1}=\int_{0}^{\infty} J \frac{\mathrm{d} P_{\geq 1}}{\mathrm{~d} J} \mathrm{~d} J=\Gamma\left(\frac{1}{1-\alpha}+1\right) \times J_{\lim }, \\
& \widetilde{J}^{\star} \approx \widetilde{J}_{\geq 1}=\log (2)^{1 /(1-\alpha)} \times J_{\lim } .
\end{aligned}
$$

The probability distribution Eq. (B.4) is defined for $\alpha>1$ and always positively skewed, even w.r.t. to a log-normal distribution. The long tail follows a power-law proportional to $J^{-\alpha}$, and thus the mean is only defined for $\alpha>2\left(\bar{J}_{\geq 1} \rightarrow \infty\right.$ for $\left.\alpha \leq 2\right)$. The mean is also always larger than the median value. For all our models in table 1 we obtain $\alpha \gtrsim 2$, with a steepening of the $\bar{N}(>J)$ distribution at the brightest halos (see figure 3). Even if a mean brightest halo could be computed in all our models, the median can always be defined (even for $\alpha \rightarrow 2$ ) and offers a much better characterisation of the brightest object.

The success of this analytic approximation is illustrated in figure 14, where the distributions from the power-law approximation (black line) and from the direct calculation over $10^{4}$ samples (red histogram) are compared for model HIGH. The two results are in excellent agreement. As stated in the main text, the skewness means that the limits on DM clump detection are extremely sensitive to the long tail of the distribution. The quantiles defining the $68 \%$ CIs are also shown: they are used for the sensitivity calculations in section 5 . We remark that similar calculations as presented in this section recently have been carried out in the context of star cluster luminosities by [169].

\section{B.3 APS mean, median, and variance}

For a particular realisation of a point-like source count distribution, the intensity power $C_{\mathrm{P}}^{I}$ defined in Eq. (3.4) scatters. The mean number of halos $\bar{N}(>F)$ brighter than a flux $F$ behaves like $\bar{N}(>L)$, i.e. it can be approximated by a power-law. Similarly to Eq. (B.1), we define $F_{\text {lim }}$ such as $\bar{N}\left(>F_{\lim }\right)=1$, and obtain

$$
N\left(F_{\min }, F^{\star}\right)=\int_{F_{\min }}^{F_{\max }=F^{\star}}\left\langle\frac{\mathrm{d} N}{\mathrm{~d} F^{\prime}}\right\rangle \mathrm{d} F^{\prime} \approx\left(\frac{F_{\min }}{F_{\lim }}\right)^{1-\alpha}-\left(\frac{F^{\star}}{F_{\lim }}\right)^{1-\alpha},
$$




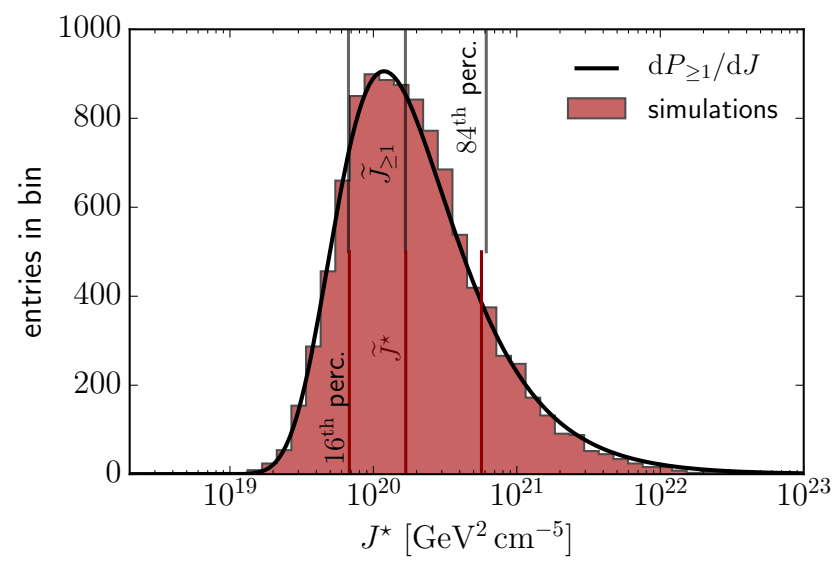

Figure 14: Probability distribution of the brightest subhalo $J^{\star}$ within the CTA survey FOV for model HIGH; $J^{\star}$ is chosen here w.r.t. to an integration angle $\theta_{\text {int }}=0.05^{\circ}$, but integrated over $\theta_{\text {vir }}$ (total emission). The histogram (red) is based on $10^{4}$ skymap realisations. The line relies on the power-law approximation $\mathrm{d} P_{\geq 1} / \mathrm{d} J$ from Eq. (B.4), with $J_{\text {lim }}=1.2 \cdot 10^{20} \mathrm{GeV}^{2} \mathrm{~cm}^{-5}$ (fixed by the $37^{\text {th }}$ percentile from the skymap realisations) and $\alpha=2.06$ (obtained from a least-square fit to the histogrammed skymap realisations). The vertical lines give the $16^{\text {th }}, 50^{\text {th }}$, and $84^{\text {th }}$ percentiles - from the skymap realisations (bottom half) or from the distribution $\mathrm{d} P \geq 1 / \mathrm{d} J$ (top half) - encompassing the $68 \%$ CI.

by integrating $\langle\mathrm{d} N / \mathrm{d} F\rangle$ up to the flux of the brightest halo $F^{\star}$. Analogously, by using the power-law $\langle\mathrm{d} N / \mathrm{d} F\rangle=(\alpha-1) / F_{\lim } \cdot\left(F / F_{\lim }\right)^{-\alpha}, \alpha>1$, and replacing $F_{\min }$ by $N$ from Eq. (B.7), one can rewrite Eq. (3.4) as

$$
C_{\mathrm{P}}^{I}\left(F^{\star}, N\right) \approx \frac{1}{4 \pi \beta} F_{\lim }^{2}\left[\left(\frac{F^{\star}}{F_{\lim }}\right)^{3-\alpha}-\left\{\left(\frac{F^{\star}}{F_{\lim }}\right)^{1-\alpha}+N\right\}^{-\beta}\right],
$$

with $\beta \equiv(3-\alpha) /(\alpha-1)$ and $\alpha \neq 3$. Thanks to this approximation, $C_{\mathrm{P}}^{I}$ now became only a function of the brightest object $F^{\star}$ and of the total number of objects $N$. A further simplification arises assuming $N \rightarrow \infty$, in which case $F_{\min } \rightarrow 0$ and Eq. (B.8) simplifies to

$$
C_{\mathrm{P}}^{I}\left(F^{\star}, N\right) \stackrel{N \rightarrow \infty}{\approx} \frac{1}{4 \pi \beta} F_{\lim }^{2}\left(\frac{F^{\star}}{F_{\lim }}\right)^{3-\alpha}
$$

Note that $C_{\mathrm{P}}^{I}$ becomes highly sensitive to a finite $N\left(F_{\min } \neq 0\right)$ in the case $\alpha \rightarrow 3$, such that the latter approximation is only applicable for $\alpha$ sufficiently smaller than 3 , and does not hold for $\alpha \geq 3$. For the case of Eq. (B.9) being valid, the median $\widetilde{C}_{\mathrm{P}}^{I}$ is directly related to $\widetilde{F}^{\star}$

$$
\widetilde{C}_{\mathrm{P}}^{I}\left(F^{\star}, N\right) \stackrel{N \rightarrow \infty}{\approx} C_{\mathrm{P}}^{I}\left(\widetilde{F}^{\star}\right) \approx \frac{1}{4 \pi \beta} F_{\lim }^{2} \log (2)^{-\beta}
$$

It is also useful (see appendix C) to fix $N \equiv \bar{N}$ in Eq. (B.8), such that $C_{\mathrm{P}}^{I}$ only becomes a function of $F^{\star}$. Then again, $\widetilde{C}_{\mathrm{P}}^{I}\left(F^{\star}\right) \approx C_{\mathrm{P}}^{I}\left(\widetilde{F}^{\star}\right)$ holds, and one can simplify the ratio of the medians to

$$
\frac{\widetilde{C}_{\mathrm{P}}^{I}\left(F^{\star} ; \bar{N}\right)}{\widetilde{C}_{\mathrm{P}}^{I}\left(F^{\star} ; N \rightarrow \infty\right)} \approx 1-\left(1+\frac{\bar{N}}{\log (2)}\right)^{-\beta} .
$$


Finally, one can extract the probability density function $\mathrm{d} P / \mathrm{d} C\left(C \equiv C_{\mathrm{P}}^{I}\right)$,

$$
\frac{\mathrm{d} P}{\mathrm{~d} C}(C)=\frac{\mathrm{d} P \geq 1}{\mathrm{~d} F}\left(F^{\star}(C)\right) \times\left|\frac{\mathrm{d} F^{\star}}{\mathrm{d} C}(C)\right|,
$$

from which one can calculate analytic expressions for the mean $\bar{C}_{\mathrm{P}}^{I}$ and standard deviation $\sigma_{C_{\mathrm{P}}}$. The latter expression shows that the PDF of $C_{\mathrm{P}}^{I}$ is proportional to the PDF of $F^{\star}$, as given in Eq. (B.4). This is in fact observed in our simulations (see figure 7), where the power at each $\ell$ shows a variance skewed w.r.t a log-normal distribution, with a long tail towards high $C_{\ell}$ values.

We stress that the above discussion only involves the variance of the APS from the varying flux of the objects. For randomly distributed objects on the sphere, the APS additionally scatters because of their random positions in space. This variance, which decreases for larger multipoles, can, on the full sky, be estimated by [170]:

$$
\sigma_{C_{\ell}}=C_{\ell} \sqrt{\frac{2}{2 \ell+1}} .
$$

\section{APS convergence}

As described in [86, 87], CLUMPY relies on a combination of the calculation of the mean signal $\left\langle J_{\text {subs }}\right\rangle$ from subhalos and the calculation of individual drawn clumps $J_{\text {drawn }}$ to ensure a quick calculation of skymaps. A critical parameter of a CLUMPY run is the relative error $R E_{J_{\text {drawn }}}$ [86], which ultimately controls the number $N_{\text {sub }}$ of clumps to be drawn. In practice, a critical distance is obtained for each mass decade, by requiring the relative error of the signal integrated from $l_{\text {crit }}$ to remain lower than this user-defined precision requirement (level of fluctuation selected w.r.t. the mean signal). This reduces the number of clumps to draw in the Galaxy from $\sim 10^{15}$ to $\sim 10^{4}$ for angular resolutions $\theta_{\text {int }} \sim 0.1^{\circ}$ and $R E_{J_{\text {drawn }}}=5 \%$. This appendix shows that this choice is a good compromise between speed and precision, and that it ensures convergence of our results to the expected values up to the highest multipoles the considered $\gamma$-ray instruments are sensitive to. The fair agreement with the approximate analytical further validates the CLUMPY code.

Figure 15 shows the ratio $\widetilde{C}_{\ell} / \widetilde{C}_{\ell}\left(N_{\text {sub }} \rightarrow \infty\right)$ for DM subhalos (model LOW) at different multipoles $\ell$, calculated over $N_{\text {sample }}=5000$ simulations. The sample median was chosen because of its robustness compared to the mean, which is ill-defined for a power law index close to $\alpha=2$. The sample size $N_{\text {sample }}=5000$ was taken in order to reach at least a $5 \%$ relative accuracy of the ratio of the medians at the lowest multipoles, as calculated by the sample error of the median (shown by the coloured area)

$$
\Delta \widetilde{C}=\frac{1}{2 \frac{\mathrm{d} P}{\mathrm{~d} C}(\widetilde{C}) \sqrt{N_{\text {sample }}}} .
$$

The APS converges differently at different multipoles, but reaches $95 \%$ of the overall power $\widetilde{C}_{\ell}\left(N_{\text {sub }} \rightarrow \infty\right)$ at all multipoles $\ell \leq 1024$ whenever $N_{\text {sub }} \gtrsim 10^{4}$ drawn objects. All power spectra presented in this work meet this requirement. For comparison purpose, the black dotted line shows the expected convergence calculated from the $\ell$-independent power-law $(\alpha=2.03)$ approximation Eq. (B.11), which gives a fair description of the sample calculation for fixed $\bar{N}_{\text {sub }}$. This approximation reveals the interesting result $\widetilde{C}_{\mathrm{P}}^{I}\left(\bar{N}_{\text {sub }}=1\right) / \widetilde{C}_{\mathrm{P}}^{I}\left(N_{\text {sub }} \rightarrow\right.$ 


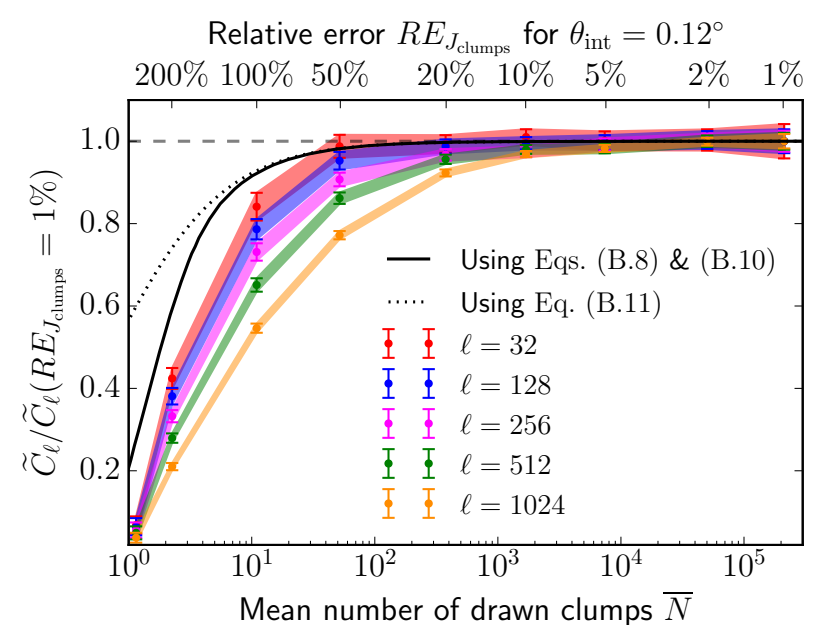

Figure 15: Convergence study of the median power $\widetilde{C}_{\ell}$ (integration angle $\theta_{\text {int }}=0.12^{\circ}$, i.e. HEALPix $N_{\text {side }}=512$ of the $J$-factor maps) as a function of $R E_{J_{\text {drawn }}}$ (upper $x$-axis) or, equivalently, the number of drawn clumps $\bar{N}_{\text {sub }}$ (lower $x$-axis). The different colours show different multipoles $\ell$ for the model LOW, with error bars from Eq. (C.1). For comparison, we also show two analytical calculations (see appendix B), based on Eq. (B.8) (black solid line) or the further approximated Eq. (B.11) (dotted line).

$\infty)=0.57$, indicating that in median, the brightest point-like object accounts for more than $50 \%$ to the overall power. The additional degree of freedom of a Poisson-distributed $N$ from sample to sample shifts down the median power for a low average $\bar{N}_{\text {sub }}$. This is accounted for in a more accurate numerical calculation of the median from Eq. (B.8), divided by Eq. (B.10), which gives an even fairer agreement with the sample median (black solid line).

\section{Virial mass and and J-factor for Milky Way satellites}

This appendix presents the derivation of $M_{\text {vir }}$ and $J\left(0.5^{\circ}\right)$ factors for spectroscopically confirmed satellite galaxies in the Milky Way. We recall that these values are used in figure 3 and figure 6 for comparisons purpose with the drawn clumps of our simulations.

Jeans and Markov Chain Monte Carlo (MCMC) analysis Most of our values come from a reprocessing the chains from the recent analyses of classical and ultrafaint dSphs of [96] for the pre-2015 dSphs, [97] for Ret II, and [99] for Tuc 2. In these papers, a Jeans analysis of the light and velocity data coupled to an MCMC engine was used to recover the DM profiles, and then extract the median values and credible intervals on several quantities deriving from these profiles (e.g., mass, $J$-factor). ${ }^{25}$ Moreover, in the last few months, and since the published results mentioned above, kinematic data of several new dSph galaxies have become publicly available. We therefore take the opportunity of this study to apply the same Jeans analysis on these dSphs, allowing for a more complete census for the objects shown in several plots of this paper. For these ultrafaint objects the number of spectroscopically measured stars is generally $\sim 5-10$. Draco II data has been taken from [171], Horologium I

\footnotetext{
${ }^{25}$ The robustness of the analysis has been validated on mock data [95]. All the tools to achieve of the steps of the analysis are public, they are described in the second release of the CLUMPY code [86, 87].
} 
from [172], Pisces II from [173] and Triangulum II from [174]. ${ }^{26}$ We do not consider in this study the faint and/or uncertain Boötes II $[176,177]$ and Hydra II $[173,178]$ dSph galaxies. We cannot exclude that several of the dSph galaxies in our selection suffer from tidal effects (see, e.g., [179] for Willman 1), but only Sagittarius dSph galaxy is undoubtedly flagged for this effect (see, e.g., [180] and references therein) and was therefore also excluded from this sample.

Tidal mass as a proxy for the virial mass As recalled in $\S 2.3$, CLUMPY works with virial quantities so that comparisons must be made w.r.t. this choice. The quantity $m_{\text {vir }}$ depends on the definition of $r_{\text {vir }}$ which, strictly speaking, depends on cosmological parameters. However,

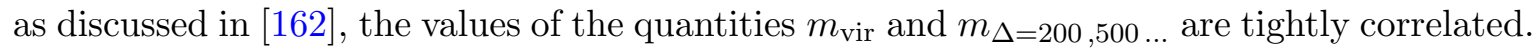
Moreover, the differences between the mass reconstructed from these various definitions is much smaller than the uncertainties we obtain from the MCMC analysis $(\sim 2$ orders of magnitude). We therefore use the mass inside the virial radius $r_{\text {vir }}$ as a measure of the total mass inside a DM halo. The authors of [58] have shown the tidal radius to be a good proxy of the virial radius, this property holding even in the presence of baryons [105]. The tidal radius is defined as [181]

$$
r_{\mathrm{tid}}=\left[\frac{m\left(r_{\mathrm{tid}}\right)}{\left[2-\mathrm{d} \ln M_{\mathrm{MW}} / \mathrm{d} \ln R\right] \times M_{\mathrm{MW}}(R)}\right]^{(1 / 3)} \times R,
$$

where $M_{\mathrm{MW}}(R)$ is the mass of the MW enclosed within the galactocentric distance $R$ of the $\mathrm{dSph}$, and $m\left(r_{\text {tid }}\right)$ is the subhalo mass inside $r_{\text {tid }}$. For each model of our MCMC analysis, we compute the enclose mass within $r_{\text {tid }}$ and define it to be $m_{\text {vir }}$ of that particular model. We underline that integrating up to $r \gg r_{\text {tid }}$ instead of $r_{\text {tid }}$ only changes by a factor of 2 the total mass, which is much smaller than the uncertainties derived from the MCMC analysis.

dSph, SMC and LMC values Table 4 gathers the distance and median (and 68\% CI) of the mass $M_{\text {vir }}$ and $J\left(0.5^{\circ}\right)$ for all the dSphs mentioned above. Given the very recent status of some of these spectroscopic measurements and the sometime intriguing kinematics they suggest (e.g., Triangulum II [175]), we urge caution in interpreting the new $J$-factors (especially the large J-factor of Triangulum II) of all the objects denoted by the star symbol $\left.{ }^{\star}\right]$ in the table. ${ }^{27}$ We also include estimation for the LMC and SMC in boldface. For these irregular objects, a spherical Jeans analysis cannot be applied, and we have taken the values from [183-185] for the LMC, and from [183, 185-187] for the SMC.

\section{E Details of the CTA analysis}

To avoid too lengthy a discussion in the core of the text, several aspects of the CTA analysis were postponed to this appendix. The key points developed here are related to the observation strategy (appendix E.1), the post-trial sensitivity (appendix E.2), the calculation of the maximum log-likelihood ratio (appendix E.3), and the robustness and values of the test statistic (appendix E.4) used in our analysis.

\footnotetext{
${ }^{26}$ Thirteen member stars in Triangulum II have recently been measured by [175] but this dataset, showing distinct behaviours in the inner and outer part of the dSph galaxy, has not been used here.

${ }^{27}$ Near the completion of this paper, the non-spherical Jeans analysis of dSph galaxies by [141] became available on arXiv and the $J$-factors of all dSph galaxies of table 4 (including the most recently discovered) are compatible at $1 \sigma$ with their values but for Segue 2 and UMa 1. Also using CLUMPY, [182] reports similar value for Triangulum II as quoted here.
} 


\begin{tabular}{|l|cc|cc|cc|}
\hline Name & $\begin{array}{c}D_{\mathrm{obs}} \\
{[\mathrm{kpc}]}\end{array}$ & $\begin{array}{r}r_{h} \\
{[\mathrm{kpc}]}\end{array}$ & \multicolumn{2}{|c|}{$\log _{10}\left[m_{\text {vir }} / \mathrm{M}_{\odot}\right]$} & \multicolumn{2}{|c|}{$\log _{10}\left[J\left(0.5^{\circ}\right) /\left(\mathrm{GeV}^{2} \mathrm{~cm}^{-5}\right)\right]$} \\
\hline${ }^{\star}$ Draco II & 20 & 0.019 & 6.80 & {$[3.63-9.20]$} & 18.1 & {$[14.9-20.5]$} \\
Segue I & 23 & 0.03 & 6.71 & {$[4.43-9.16]$} & 17.2 & {$[14.9-19.0]$} \\
Ursa Major II & 30 & 0.14 & 9.49 & {$[8.31-10.9]$} & 19.9 & {$[19.3-20.5]$} \\
Reticulum II & 30 & 0.032 & 8.95 & {$[7.46-10.7]$} & 19.5 & {$[18.8-20.5]$} \\
${ }^{\star}$ Triangulum II & 30 & 0.034 & 8.90 & {$[7.26-11.1]$} & 20.9 & {$[19.7-22.3]$} \\
Segue II & 35 & 0.03 & 8.87 & {$[7.13-10.5]$} & 18.9 & {$[17.8-20.0]$} \\
Willman I & 38 & 0.02 & 8.26 & {$[7.08-10.3]$} & 19.5 & {$[18.9-20.7]$} \\
Coma & 44 & 0.08 & 9.45 & {$[8.05-10.9]$} & 19.6 & {$[18.9-20.4]$} \\
Tucana II & 57 & 0.165 & 8.72 & {$[7.38-10.3]$} & 18.6 & {$[17.7-19.5]$} \\
Ursa Minor & 66 & 0.28 & 8.64 & {$[8.31-9.24]$} & 19.0 & {$[18.9-19.1]$} \\
Boötes I & 66 & 0.24 & 8.95 & {$[8.13-10.0]$} & 18.5 & {$[18.1-19.2]$} \\
Sculptor & 79 & 0.26 & 8.29 & {$[8.06-8.68]$} & 18.5 & {$[18.5-18.7]$} \\
${ }^{\star}$ Horologium I & 79 & 0.03 & 8.84 & {$[7.06-10.9]$} & 19.8 & {$[18.8-21.3]$} \\
Draco & 82 & 0.20 & 9.62 & {$[8.85-10.5]$} & 19.1 & {$[18.8-19.5]$} \\
Sextans & 86 & 0.68 & 9.02 & {$[8.57-9.68]$} & 17.6 & {$[17.4-17.8]$} \\
Ursa Major I & 97 & 0.32 & 9.13 & {$[8.34-10.2]$} & 18.7 & {$[18.3-19.3]$} \\
Carina & 101 & 0.24 & 8.68 & {$[8.16-9.35]$} & 17.9 & {$[17.8-18.1]$} \\
Hercules & 132 & 0.33 & 8.93 & {$[8.24-9.92]$} & 17.5 & {$[16.8-18.2]$} \\
Fornax & 138 & 0.67 & 8.93 & {$[8.67-9.26]$} & 17.7 & {$[17.6-17.8]$} \\
Leo IV & 160 & 0.11 & 7.87 & {$[6.61-9.29]$} & 16.2 & {$[14.6-17.5]$} \\
Canes Venatici II & 160 & 0.07 & 9.38 & {$[8.10-10.8]$} & 18.5 & {$[17.6-19.7]$} \\
Leo V & 180 & 0.03 & 8.11 & {$[6.88-9.38]$} & 16.1 & {$[15.0-17.3]$} \\
^Pisces II & 182 & 0.058 & 7.75 & {$[6.35-9.72]$} & 16.9 & {$[15.2-18.4]$} \\
Leo II & 205 & 0.14 & 9.06 & {$[8.37-10.0]$} & 18.0 & {$[17.8-18.6]$} \\
Canes Venatici I & 218 & 0.57 & 9.20 & {$[8.74-9.92]$} & 17.5 & {$[17.3-17.9]$} \\
Leo I & 250 & 0.24 & 9.41 & {$[8.63-10.2]$} & 17.8 & {$[17.6-18.3]$} \\
LeoT & 407 & 0.18 & 9.12 & {$[8.27-10.4]$} & 17.6 & {$[17.1-18.6]$} \\
\hline & & Type & Central & Min/Max & Central & Min/Max \\
LMC & 50 & SBm & 10.5 & $10.2 / 11.4$ & 19.8 & $19.1 / 21.7$ \\
SMC & 62 & dIrr & 9.81 & $9.43 / 10.6$ & 18.4 & $16.3 / 18.6$ \\
\hline
\end{tabular}

Table 4: The Galaxy satellites are ordered by distance and the columns correspond to (from left to right): name, distance, typical half-light radius (for dSphs) or type (for Magellanic clouds), median and $68 \%$ CI virial mass, median and $68 \%$ CI $J$-factor in an integration angle of $0.5^{\circ}$. In our calculations, we do not account for the uncertainties on the distance $D_{\text {obs }}$ and the half-light radius $r_{h}$ of the $\mathrm{dSph}$ galaxies, and only quote the values we have actually used in the analysis. Objects in italic are confirmed ultrafaint dSphs while the ones denoted by the star symbol [^] are the most recently discovered objects, tentatively analysed here. The boldface objects correspond to the large and small Magellanic clouds, for which the central and Min/Max values represent the range of modelling uncertainties taken from the literature (see text).

\section{E.1 Observation strategy}

For isotropically distributed sources on the sky, one may ask if a large field survey with a shallow exposure is the most sensitive strategy to detect these objects. It has been shown 
that observing a small portion of the sky with the same available total time is not beneficial for most source populations, and the loss of chance to encounter a very bright object within a small field of view outweighs the gain in deep-exposure sensitivity [85]. We show below that this also applies to Galactic DM subhalos isotropically distributed on large scales. The isotropy assumption does not strictly hold for model HIGH, but still yields a fair characterisation of the subhalo abundance.

We assume isotropically distributed sources on the sky obeying a power-law source count distribution, Eq. (B.1). Then one obtains for the mean number $\bar{N}$ of subhalos with a flux above a given threshold and on a survey area $A$ :

$$
\bar{N}(>F, A)=\left(\frac{F}{F_{\text {lim }, \text { fullsky }}}\right)^{1-\alpha} \frac{A}{4 \pi \mathrm{sr}} .
$$

so that

$$
\frac{\bar{N}\left(>F_{1}, A_{1}\right)}{\bar{N}\left(>F_{2}, A_{2}\right)}=\left(\frac{F_{1}}{F_{2}}\right)^{1-\alpha} \frac{A_{1}}{A_{2}} .
$$

For a fixed total observation time $T$ available to uniformly cover an area of the total size $A$, then an area within $A_{\mathrm{FOV}}$ can be observed for $t=T \times\left(A_{\mathrm{FOV}} / A\right)$. As the sensitivity to a flux $F$ goes as $1 / \sqrt{t}$, we have $F_{1} / F_{2}=\sqrt{t_{2} / t_{1}}$, and $t_{1} / t_{2}=A_{2} / A_{1}$, so that

$$
\frac{\bar{N}_{\text {detectable }}\left(A_{1}, T\right)}{\bar{N}_{\text {detectable }}\left(A_{2}, T\right)}=\left(\frac{A_{1}}{A_{2}}\right)^{\frac{3-\alpha}{2}},
$$

with $A_{1}$ and $A_{2}$ the observed area on the sky and $\bar{N}_{\text {detectable }}$ the mean number of detectable objects. Thus, for a power-law index $\alpha<3$, the average number of detectable subhalos is $\bar{N}\left(A_{1}\right)>\bar{N}\left(A_{2}\right)$ for $A_{1}>A_{2}$ independent of $T$, and the probability of detecting an object from the population rises for increasing the survey area. All our subhalo models meet this requirement, with $2 \lesssim \alpha<3$. It is also useful to extract from Eq. (E.1) the relation

$$
\frac{F_{\lim , \mathrm{A}_{1}}}{F_{\lim , \mathrm{A}_{2}}}=\left(\frac{A_{1}}{A_{2}}\right)^{\frac{1}{\alpha-1}} .
$$

For a power-law distribution, according to Eq. (B.5) and (B.6), this ratio also holds for the mean and median brightest halo,

$$
\frac{F_{\lim , \mathrm{A}_{1}}}{F_{\lim , \mathrm{A}_{2}}}=\frac{\bar{F}_{A_{1}}^{\star}}{\bar{F}_{A_{2}}^{\star}}=\frac{\widetilde{F}_{A_{1}}^{\star}}{\widetilde{F}_{A_{2}}^{\star}} .
$$

For a power-law index $\alpha \approx 2$, the ratio of mean/median fluxes ( $J$-factors) of the brightest halo within two survey fields $A_{1}, A_{2}$ is proportional to the ratio of the field sizes. We remark that this result also applies to the APS behaviour discussed in appendix B.3. Adopting Eq. (B.10), one obtains for $\alpha \approx 2$

$$
\widetilde{C}_{\mathrm{P}}^{I}\left(A_{1}\right) \approx\left(\frac{A_{1}}{A_{2}}\right)^{2} \widetilde{C}_{\mathrm{P}}^{I}\left(A_{2}\right) .
$$

For $A_{2}=4 \pi \mathrm{sr}$, it is $A_{1} / A_{2}=f_{\text {sky }}$, the fraction of the sky. Note the $f_{\text {sky }}^{2}$ scaling of Eq. (E.6) in the latter case. 


\section{E.2 Pre- and post-trial sensitivity}

To realistically assess a survey sensitivity, one has to account for the trials when searching for a signal from unspecified locations. Assuming an average $68 \%$ containment radius of the CTA point spread function of $0.05^{\circ}$, corresponding to a containment area of $2.4 \cdot 10^{-6} \mathrm{sr}$, scanning a quarter of the sky results in approximately $\pi /\left(2.4 \cdot 10^{-6}\right)=1.3 \cdot 10^{6}$ independent trials. $^{28}$ In order to reject a background fluctuation in the survey search at a trials corrected confidence level $1-p_{\text {post }}$, we calculate the required confidence level, $1-p_{\text {pre }}$, for the template observation setup according to

$$
p_{\text {pre }}=1-\left(1-p_{\text {post }}\right)^{1 / N_{\text {trials }}} .
$$

Presenting our results at the $1-p_{\text {post }}=95 \%$ confidence level, with $N_{\text {trials }}=1.3 \cdot 10^{6}$, we thus require $p_{\text {pre }}=3.9 \cdot 10^{-8}$. This corresponds to a Gaussian one-sided confidence level, $p=\alpha / 2$, of $5.4 \sigma$.

\section{E.3 Likelihood}

The full unbinned likelihood function for a specific model $\mathcal{M}$ considered in our study is

$$
\mathscr{L}\left(\mathcal{M} \mid N_{\text {obs }}, E_{\text {obs }, 1 \ldots N_{\text {obs }}}, \vec{k}_{\text {obs }, 1 \ldots N_{\text {obs }}}\right)=p\left(N_{\text {obs }} \mid N_{\text {pred }}(\mathcal{M})\right) \times \prod_{1}^{N_{\text {obs }}} p\left(E_{\text {obs }, \mathrm{i}}, \vec{k}_{\text {obs }, \mathrm{i}} \mid \mathcal{M}\right) .
$$

Here, $N_{\text {obs }}$ is the total number of observed events, $E_{\mathrm{obs}, \mathrm{i}}$ and $\vec{k}_{\mathrm{obs}, \mathrm{i}}$ denote the reconstructed energy and angular direction of each event. For Poisson statistics,

$$
p\left(N_{\text {obs }} \mid N_{\text {pred }}\right)=\frac{N_{\text {pred }}^{N_{\text {obs }}} e^{-N_{\text {pred }}}}{N_{\text {obs }} !} .
$$

The total number of predicted events, $N_{\text {pred }}$, is calculated by

$$
N_{\text {pred }}(\mathcal{M})=T_{\text {obs }} \int_{E_{\min }=30 \mathrm{GeV}}^{E_{\max }=200 \mathrm{TeV}} \int_{\Delta \Omega_{\mathrm{obs}}} p\left(E_{\text {obs }}, \vec{k}_{\text {obs }} \mid \mathcal{M}\right) \mathrm{d} E_{\text {obs }} \mathrm{d} \Omega_{\text {obs }}
$$

with $T_{\text {obs }}$ the duration of the observation. ${ }^{29}$ The probability $p\left(E_{\text {obs }}, \vec{k}_{\text {obs }} \mid \mathcal{M}\right)$ for each event is given by the differential intensity $\mathrm{d} \Phi_{\mathcal{M}} /(\mathrm{d} E \mathrm{~d} \Omega)$ predicted by the model $\mathcal{M}$, integrated over the effective area, $A_{\text {eff }}$, and convolved with the energy and angular response of the instrument,

$$
p\left(E_{\mathrm{obs}}, \vec{k}_{\mathrm{obs}} \mid \mathcal{M}\right)=\int_{E, \Omega, A_{\text {eff }}(E)} p\left(E_{\mathrm{obs}} \mid E, \vec{k}\right) \times p\left(\vec{k}_{\mathrm{obs}} \mid E, \vec{k}\right) \times \frac{\mathrm{d} \Phi_{\mathcal{M}}}{\mathrm{d} E \mathrm{~d} \Omega}(E, \vec{k}) \mathrm{d} A \mathrm{~d} E \mathrm{~d} \Omega,
$$

with $E$ and $\vec{k}$ the true energy and direction of the event. ${ }^{30}$ For computation reasons, we neglect the energy dispersion of the events, i.e. we set $p\left(E_{\mathrm{obs}} \mid E, \vec{k}\right)=\delta\left(E-E_{\mathrm{obs}}\right)$, whereas

\footnotetext{
${ }^{28}$ By chance, this number comes close to the frequency one expects a $5 \sigma$ background up-fluctuation in $1 / p=3.5 \cdot 10^{6}$ repetitions of a random experiment.

${ }^{29}$ We do not include the time coordinates of the events into the likelihood calculation, as both the event rates from background and DM annihilation are assumed to be constant in time.

${ }^{30}$ In general, the effective area additionally depends on the time-dependent zenith and azimuthal coordinates of the observation, i.e. $A_{\text {eff }}=A_{\text {eff }}(E, \vec{k}, t)$, which would introduce a time dependence to $p\left(E_{\text {obs }}, \vec{k}_{\text {obs }}, t \mid \mathcal{M}\right)$ and to the likelihood Eq. (E.8). However, for simplicity, we perform our study with a constant $A_{\text {eff }}$.
} 


\begin{tabular}{|c|ccc|ccc|}
\hline & \multicolumn{3}{|c|}{$p_{\text {pre }}=0.05(1.6 \sigma)$} & \multicolumn{3}{c|}{$p_{\text {pre }}=3.9 \cdot 10^{-8}(5.4 \sigma)$} \\
$m_{\chi}$ range $[\mathrm{GeV}]$ & $\chi \chi \rightarrow b \bar{b}$ & $\chi \chi \rightarrow \tau^{+} \tau^{-}$ & $0.5 \chi_{k=1}^{2}$ & $\chi \chi \rightarrow b \bar{b}$ & $\chi \chi \rightarrow \tau^{+} \tau^{-}$ & $0.5 \chi_{k=1}^{2}$ \\
\hline $50-100$ & 1.5 & 1.5 & 2.71 & 24 & 28 & 29.1 \\
$150-500$ & 2.5 & 2.9 & 2.71 & 31 & 32 & 29.1 \\
$600-1000$ & 3.4 & 2.5 & 2.71 & 35 & 30 & 29.1 \\
$1500-7500$ & 3.0 & 1.9 & 2.71 & 34 & 28 & 29.1 \\
$10^{4}-10^{5}$ & 2.0 & 1.1 & 2.71 & 29 & 26 & 29.1 \\
\hline
\end{tabular}

Table 5: Test statistic values used for the CTA sensitivity analysis in this study. The values given in the left and middle columns $(\chi \chi \rightarrow X X)$ are obtained from our MC calculation, the right columns are expected from Wilks' theorem $\left(0.5 \chi_{k=1}^{2}\right)$. The pre-trial p-value from the left block results into a post-trial p-value of 0.05 for $N_{\text {trials }}=1.3 \cdot 10^{6}$. See appendix E.2 for pre- and post-trial definitions.

the point spread function, $p\left(\vec{k}_{\text {obs }} \mid E, \vec{k}\right)$, is modelled as a two-dimensional Gaussian with energy-dependent width $\sigma(E)$.

For the signal model $\mathcal{M}_{\text {sig }}, \mathrm{d} \Phi_{\mathcal{M}_{\text {sig }}} /(\mathrm{d} E \mathrm{~d} \Omega)$ is the $\gamma$-ray intensity from DM annihilation, given by Eq. (2.1). For the background model $\mathcal{M}_{\mathrm{bkg}}, \mathrm{d} \Phi_{\mathcal{M}_{\mathrm{bkg}}} /(\mathrm{d} E \mathrm{~d} \Omega)$ corresponds to the residual cosmic ray background after cuts, and we directly make use of the background rate model shown in figure 11, so that ${ }^{31}$

$$
p\left(E_{\text {obs }}, \vec{k}_{\text {obs }} \mid \mathcal{M}_{\text {bkg }}\right)=\frac{\mathrm{d} N_{\text {bkg }}}{\mathrm{d} E_{\text {obs }} \mathrm{d} \Omega_{\text {obs }} \mathrm{d} t}=f\left(E_{\text {obs }}, \vec{k}_{\text {obs }}\right) .
$$

\section{E.4 Distribution and values of the test statistic (TS)}

For the test statistic $\lambda$ defined in Eq. (5.2), the hypothesis $\mathcal{M}_{\mathrm{bkg}}+\mathcal{M}_{\text {sig }}$ has one more degree of freedom than the hypothesis background only. In the limit of a large data sample (event number) and provided the physical bound $\langle\sigma v\rangle \geq 0$, TS is expected to be distributed according to $[188,189]$

$$
p(\mathrm{TS})=\frac{1}{2} \delta(\mathrm{TS})+\frac{1}{2} \chi_{k=1}^{2}(\mathrm{TS}) .
$$

However, we found from $10^{5} \mathrm{MC}$ simulations that for our setup, the TS distribution is poorly described by Eq. (E.13), and depends on the spectral shape of $\mathcal{M}_{\text {sig. }}$. Table 5 shows the used test statistic (TS) values for the sensitivity calculation in section 5 . We performed $N_{\text {sim }}=10^{5} \mathrm{MC}$ simulations distributed over 24 spectra. We then merged the spectra into five groups, and calculated $\operatorname{TS}\left(p_{\text {pre }}=0.05\right)$ separately for each group. Because performing $10^{8} \mathrm{MC}$ simulations for $p_{\text {pre }}=3.9 \cdot 10^{-8}$ was not feasible, we fitted an exponential tail to our distributions, and computed $\operatorname{TS}\left(p_{\text {pre }}=3.9 \cdot 10^{-8}\right)$ from this extrapolation. Table 5 shows that the obtained TS values (left and middle columns) approximately correspond to the assumption of a $\chi_{k=1}^{2}$ distribution (right columns, constant and independent of the DM spectrum), but deviate for very low and high DM masses.

\section{References}

[1] J. E. Gunn, B. W. Lee, I. Lerche, D. N. Schramm and G. Steigman, Some astrophysical consequences of the existence of a heavy stable neutral lepton, ApJ 223 (Aug., 1978) $1015-1031$.

\footnotetext{
${ }^{31}$ The background rate model is taken for the chosen $A_{\text {eff }}$ and thus is also constant in time for our study.
} 
[2] F. W. Stecker, The cosmic $\gamma$-ray background from the annihilation of primordial stable neutral heavy leptons, ApJ 223 (Aug., 1978) 1032-1036.

[3] L. Bergström, Dark matter candidates, New Journal of Physics 11 (Oct., 2009) 105006, [0903.4849].

[4] J. Silk and H. Bloemen, A $\gamma$-ray constraint on the nature of dark matter, ApJ 313 (Feb., 1987) L47-L51.

[5] F. W. Stecker, Gamma ray constraints on dark matter reconsidered, Physics Letters B 201 (Feb., 1988) 529-532.

[6] F. Aharonian et al., Very high energy gamma rays from the direction of Sagittarius $A^{*}, A \mathscr{E} A$ 425 (Oct., 2004) L13-L17, [arXiv: astro-ph/0406658].

[7] D. Horns, TeV $\gamma$-radiation from Dark Matter annihilation in the Galactic center, Physics Letters B 607 (Feb., 2005) 225-232, [astro-ph/0408192].

[8] G. Lake, High dark matter densities and the formation of extreme dwarf galaxies, ApJ $\mathbf{3 5 6}$ (June, 1990) L43-L46.

[9] G. Lake, Detectability of gamma-rays from clumps of dark matter, Nature 346 (July, 1990) 39.

[10] J. Silk and A. Stebbins, Clumpy cold dark matter, ApJ 411 (July, 1993) 439-449.

[11] L. Bergström, P. Ullio and J. H. Buckley, Observability of $\gamma$ rays from dark matter neutralino annihilations in the Milky Way halo, Astropart. Phys. 9 (Aug., 1998) 137-162, [astro-ph/9712318].

[12] V. Berezinsky, V. Dokuchaev and Y. Eroshenko, Small-scale clumps in the galactic halo and dark matter annihilation, Phys. Rev. D 68 (Nov., 2003) 103003, [astro-ph/0301551].

[13] C. Calcáneo-Roldán and B. Moore, Surface brightness of dark matter: Unique signatures of neutralino annihilation in the galactic halo, Phys. Rev. D 62 (Dec., 2000) 123005, [astro-ph/0010056].

[14] A. Tasitsiomi and A. V. Olinto, Detectability of neutralino clumps via atmospheric Cherenkov telescopes, Phys. Rev. D 66 (Oct., 2002) 083006, [astro-ph/0206040].

[15] W. B. Atwood, A. A. Abdo, M. Ackermann, W. Althouse, B. Anderson, M. Axelsson et al., The Large Area Telescope on the Fermi Gamma-Ray Space Telescope Mission, ApJ 697 (June, 2009) 1071-1102, [0902.1089].

[16] J. Conrad, J. Cohen-Tanugi and L. E. Strigari, Wimp searches with gamma rays in the Fermi era: Challenges, methods and results, Soviet Journal of Experimental and Theoretical Physics 121 (Dec., 2015) 1104-1135, [1503.06348].

[17] A. A. Abdo, M. Ackermann, M. Ajello, W. B. Atwood, L. Baldini, J. Ballet et al., Fermi Large Area Telescope Search for Photon Lines from 30 to $200 \mathrm{GeV}$ and Dark Matter Implications, Phys. Rev. Lett. 104 (Mar., 2010) 091302, [1001.4836].

[18] M. Ackermann, M. Ajello, A. Albert, L. Baldini, G. Barbiellini, K. Bechtol et al., Fermi LAT search for dark matter in gamma-ray lines and the inclusive photon spectrum, Phys. Rev. D 86 (July, 2012) 022002, [1205.2739].

[19] M. Ackermann, M. Ajello, A. Albert, A. Allafort, L. Baldini, G. Barbiellini et al., Search for gamma-ray spectral lines with the Fermi Large Area Telescope and dark matter implications, Phys. Rev. D 88 (Oct., 2013) 082002.

[20] M. Ackermann, M. Ajello, A. Albert, B. Anderson, W. B. Atwood, L. Baldini et al., Updated search for spectral lines from Galactic dark matter interactions with pass 8 data from the Fermi Large Area Telescope, Phys. Rev. D 91 (June, 2015) 122002. 
[21] A. A. Abdo, M. Ackermann, M. Ajello, L. Baldini, J. Ballet, G. Barbiellini et al., Constraints on cosmological dark matter annihilation from the Fermi-LAT isotropic diffuse gamma-ray measurement, J. Cosmology Astropart. Phys. 4 (Apr., 2010) 014, [1002.4415].

[22] M. Ackermann, M. Ajello, W. B. Atwood, L. Baldini, G. Barbiellini, D. Bastieri et al., Constraints on the Galactic Halo Dark Matter from Fermi-LAT Diffuse Measurements, ApJ 761 (Dec., 2012) 91, [1205.6474].

[23] The Fermi LAT Collaboration, Limits on dark matter annihilation signals from the Fermi LAT 4-year measurement of the isotropic gamma-ray background, J. Cosmology Astropart. Phys. 9 (Sept., 2015) 008, [1501.05464].

[24] M. Ackermann, M. Ajello, A. Allafort, L. Baldini, J. Ballet, G. Barbiellini et al., Constraints on dark matter annihilation in clusters of galaxies with the Fermi large area telescope, J. Cosmology Astropart. Phys. 5 (May, 2010) 025, [1002.2239].

[25] M. Ackermann, M. Ajello, A. Albert, W. B. Atwood, L. Baldini, G. Barbiellini et al., Search for Extended Gamma-Ray Emission from the Virgo Galaxy Cluster with FERMI-LAT, ApJ 812 (Oct., 2015) 159, [1510.00004].

[26] A. A. Abdo, M. Ackermann, M. Ajello, W. B. Atwood, L. Baldini, J. Ballet et al., Observations of Milky Way Dwarf Spheroidal Galaxies with the Fermi-Large Area Telescope Detector and Constraints on Dark Matter Models, ApJ 712 (Mar., 2010) 147-158, [1001.4531].

[27] M. Ackermann, M. Ajello, A. Albert, W. B. Atwood, L. Baldini, J. Ballet et al., Constraining Dark Matter Models from a Combined Analysis of Milky Way Satellites with the Fermi Large Area Telescope, Phys. Rev. Lett. 107 (Dec., 2011) 241302, [1108.3546].

[28] M. Ackermann, A. Albert, L. Baldini, J. Ballet, G. Barbiellini, D. Bastieri et al., Search for Dark Matter Satellites Using Fermi-LAT, ApJ 747 (Mar., 2012) 121, [1201.2691].

[29] M. Ackermann, A. Albert, B. Anderson, L. Baldini, J. Ballet, G. Barbiellini et al., Dark matter constraints from observations of 25 Milky $\hat{A}$ Way satellite galaxies with the Fermi Large Area Telescope, Phys. Rev. D 89 (Feb., 2014) 042001, [1310.0828].

[30] M. Ackermann, A. Albert, B. Anderson, W. B. Atwood, L. Baldini, G. Barbiellini et al., Searching for Dark Matter Annihilation from Milky Way Dwarf Spheroidal Galaxies with Six Years of Fermi Large Area Telescope Data, Phys. Rev. Lett. 115 (Dec., 2015) 231301, [1503.02641].

[31] A. Drlica-Wagner, A. Albert, K. Bechtol, M. Wood, L. Strigari, M. Sánchez-Conde et al., Search for Gamma-Ray Emission from DES Dwarf Spheroidal Galaxy Candidates with Fermi-LAT Data, ApJ 809 (Aug., 2015) L4, [1503.02632].

[32] A. Abramowski, F. Acero, F. Aharonian, A. G. Akhperjanian, G. Anton, A. Barnacka et al., Search for a Dark Matter Annihilation Signal from the Galactic Center Halo with H.E.S.S., Phys. Rev. Lett. 106 (Apr., 2011) 161301, [1103.3266].

[33] A. Abramowski, F. Aharonian, F. Ait Benkhali, A. G. Akhperjanian, E. O. Angüner, M. Backes et al., Constraints on an Annihilation Signal from a Core of Constant Dark Matter Density around the Milky Way Center with H.E.S.S., Phys. Rev. Lett. 114 (Feb., 2015) 081301, [1502.03244].

[34] V. A. Acciari, T. Arlen, T. Aune, M. Beilicke, W. Benbow, D. Boltuch et al., VERITAS Search for VHE Gamma-ray Emission from Dwarf Spheroidal Galaxies, ApJ 720 (Sept., 2010) 1174-1180, [1006.5955].

[35] B. f. Zitzer, Search for Dark Matter from Dwarf Galaxies using VERITAS, ArXiv e-prints (Sept., 2015), [1509.01105]. 
[36] A. Abramowski, F. Aharonian, F. Ait Benkhali, A. G. Akhperjanian, E. Angüner, M. Backes et al., Search for dark matter annihilation signatures in H.E.S.S. observations of dwarf spheroidal galaxies, Phys. Rev. D 90 (Dec., 2014) 112012, [1410.2589].

[37] J. Aleksić, S. Ansoldi, L. A. Antonelli, P. Antoranz, A. Babic, P. Bangale et al., Optimized dark matter searches in deep observations of Segue 1 with MAGIC, J. Cosmology Astropart. Phys. 2 (Feb., 2014) 008, [1312.1535].

[38] MAGIC collaboration, Limits to dark matter annihilation cross-section from a combined analysis of MAGIC and Fermi-LAT observations of dwarf satellite galaxies, J. Cosmology Astropart. Phys. 2 (Feb., 2016) 039, [1601.06590].

[39] M. Ajello, A. Albert, W. B. Atwood, G. Barbiellini, D. Bastieri, K. Bechtol et al., Fermi-LAT Observations of High-Energy Gamma-Ray Emission toward the Galactic Center, ApJ $\mathbf{8 1 9}$ (Mar., 2016) 44, [1511.02938].

[40] G. Battaglia, A. Helmi and M. Breddels, Internal kinematics and dynamical models of dwarf spheroidal galaxies around the Milky Way, New A Rev. 57 (Sept., 2013) 52-79, [1305.5965].

[41] L. E. Strigari, Galactic searches for dark matter, Phys. Rep. 531 (Oct., 2013) 1-88, [1211.7090].

[42] T. E. Jeltema, J. Kehayias and S. Profumo, Gamma rays from clusters and groups of galaxies: Cosmic rays versus dark matter, Phys. Rev. D 80 (July, 2009) 023005, [0812.0597].

[43] Q. Yuan, P.-F. Yin, X.-J. Bi, X.-M. Zhang and S.-H. Zhu, Gamma rays and neutrinos from dark matter annihilation in galaxy clusters, Phys. Rev. D 82 (July, 2010) 023506, [1002.0197].

[44] A. Pinzke, C. Pfrommer and L. Bergström, Prospects of detecting gamma-ray emission from galaxy clusters: Cosmic rays and dark matter annihilations, Phys. Rev. D 84 (Dec., 2011) 123509, [1105.3240].

[45] S. Ando and D. Nagai, Fermi-LAT constraints on dark matter annihilation cross section from observations of the Fornax cluster, J. Cosmology Astropart. Phys. 7 (July, 2012) 017, [1201.0753].

[46] E. Nezri, R. White, C. Combet, J. A. Hinton, D. Maurin and E. Pointecouteau, $\gamma$-rays from annihilating dark matter in galaxy clusters: stacking versus single source analysis, MNRAS 425 (Sept., 2012) 477-489, [1203.1165].

[47] M. Fornasa and M. A. Sánchez-Conde, The nature of the Diffuse Gamma-Ray Background, Phys. Rep. 598 (Oct., 2015) 1-58, [1502.02866].

[48] V. S. Berezinsky, A. V. Gurevich and K. P. Zybin, Distribution of dark matter in the galaxy and the lower limits for the masses of supersymmetric particles, Physics Letters B 294 (Nov., 1992) 221-228.

[49] S. Colafrancesco, P. Marchegiani and G. Beck, Evolution of dark matter halos and their radio emissions, J. Cosmology Astropart. Phys. 2 (Feb., 2015) 032, [1409.4691].

[50] K. Spekkens, B. S. Mason, J. E. Aguirre and B. Nhan, A Deep Search for Extended Radio Continuum Emission from Dwarf Spheroidal Galaxies: Implications for Particle Dark Matter, ApJ 773 (Aug., 2013) 61, [1301.5306].

[51] A. Natarajan, J. B. Peterson, T. C. Voytek, K. Spekkens, B. Mason, J. Aguirre et al., Bounds on dark matter properties from radio observations of Ursa Major II using the Green Bank Telescope, Phys. Rev. D 88 (Oct., 2013) 083535, [1308.4979].

[52] M. Regis, S. Colafrancesco, S. Profumo, W. J. G. de Blok, M. Massardi and L. Richter, Local Group dSph radio survey with ATCA (III): constraints on particle dark matter, J. Cosmology Astropart. Phys. 10 (Oct., 2014) 016, [1407.4948]. 
[53] S. Colafrancesco, S. Profumo and P. Ullio, Multi-frequency analysis of neutralino dark matter annihilations in the Coma cluster, A\&BA 455 (Aug., 2006) 21-43, [arXiv:astro-ph/0507575].

[54] E. Storm, T. E. Jeltema, S. Profumo and L. Rudnick, Constraints on Dark Matter Annihilation in Clusters of Galaxies from Diffuse Radio Emission, ApJ 768 (May, 2013) 106, [1210.0872].

[55] N. Fornengo and M. Regis, Particle dark matter searches in the anisotropic sky, Frontiers in Physics 2 (Feb., 2014) 6, [1312.4835].

[56] M. Kuhlen, M. Vogelsberger and R. Angulo, Numerical simulations of the dark universe: State of the art and the next decade, Physics of the Dark Universe 1 (Nov., 2012) 50-93, [1209.5745].

[57] J. Diemand, M. Kuhlen and P. Madau, Dark Matter Substructure and Gamma-Ray Annihilation in the Milky Way Halo, ApJ 657 (Mar., 2007) 262-270, [astro-ph/0611370].

[58] V. Springel, J. Wang, M. Vogelsberger, A. Ludlow, A. Jenkins, A. Helmi et al., The Aquarius Project: the subhaloes of galactic haloes, MNRAS 391 (Dec., 2008) 1685-1711, [0809.0898].

[59] W. A. Hellwing, C. S. Frenk, M. Cautun, S. Bose, J. Helly, A. Jenkins et al., The Copernicus Complexio: a high-resolution view of the small-scale Universe, MNRAS 457 (Apr., 2016) 3492-3509, [1505.06436].

[60] S. Profumo, K. Sigurdson and M. Kamionkowski, What Mass Are the Smallest Protohalos?, Phys. Rev. Lett. 97 (July, 2006) 031301, [astro-ph/0603373].

[61] J. Diemand, B. Moore and J. Stadel, Earth-mass dark-matter haloes as the first structures in the early Universe, Nature 433 (Jan., 2005) 389-391, [astro-ph/0501589].

[62] V. S. Berezinsky, V. I. Dokuchaev and Y. N. Eroshenko, Small-scale clumps of dark matter, Physics Uspekhi 57 (Jan., 2014) 1-36, [1405.2204].

[63] F. Stoehr, S. D. M. White, V. Springel, G. Tormen and N. Yoshida, Dark matter annihilation in the halo of the Milky Way, MNRAS 345 (Nov., 2003) 1313-1322, [astro-ph/0307026].

[64] M. Kuhlen, J. Diemand and P. Madau, The Dark Matter Annihilation Signal from Galactic Substructure: Predictions for GLAST, ApJ 686 (Oct., 2008) 262-278, [0805.4416].

[65] M. Kuhlen, P. Madau and J. Silk, Exploring Dark Matter with Milky Way Substructure, Science 325 (Aug., 2009) 970, [0907.0005].

[66] B. Anderson, M. Kuhlen, J. Diemand, R. P. Johnson and P. Madau, Fermi-LAT Sensitivity to Dark Matter Annihilation in VIA Lactea II Substructure, ApJ 718 (Aug., 2010) 899-904, [1006.1628].

[67] L. Pieri, J. Lavalle, G. Bertone and E. Branchini, Implications of high-resolution simulations on indirect dark matter searches, Phys. Rev. D 83 (Jan., 2011) 023518, [0908.0195].

[68] S. M. Koushiappas, A. R. Zentner and T. P. Walker, Observability of gamma rays from neutralino annihilations in the Milky Way substructure, Phys. Rev. D 69 (Feb., 2004) 043501, [astro-ph/0309464].

[69] L. Pieri and E. Branchini, Dark matter annihilation in the local group, Phys. Rev. D 69 (Feb., 2004) 043512, [astro-ph/0307209].

[70] T. Oda, T. Totani and M. Nagashima, Gamma-Ray Background from Neutralino Annihilation in the First Cosmological Objects, ApJ 633 (Nov., 2005) L65-L68, [astro-ph/0504096].

[71] L. Pieri, G. Bertone and E. Branchini, Dark matter annihilation in substructures revised, MNRAS 384 (Mar., 2008) 1627-1637, [0706.2101].

[72] S. K. Lee, S. Ando and M. Kamionkowski, The gamma-ray-flux PDF from galactic halo substructure, J. Cosmology Astropart. Phys. 7 (July, 2009) 007, [0810.1284]. 
[73] S. M. Koushiappas, A. R. Zentner and A. V. Kravtsov, Distribution of annihilation luminosities in dark matter substructure, Phys. Rev. D 82 (Oct., 2010) 083504, [1006.2391].

[74] H.-S. Zechlin, M. V. Fernandes, D. Elsässer and D. Horns, Dark matter subhaloes as gamma-ray sources and candidates in the first Fermi-LAT catalogue, A $\mathscr{J} A \mathbf{5 3 8}$ (Feb., 2012) A93, [1111.3514].

[75] H.-S. Zechlin and D. Horns, Unidentified sources in the Fermi-LAT second source catalog: the case for DM subhalos, J. Cosmology Astropart. Phys. 11 (Nov., 2012) 050, [1210. 3852].

[76] A. V. Belikov, M. R. Buckley and D. Hooper, Searching for dark matter subhalos in the Fermi-LAT second source catalog, Phys. Rev. D 86 (Aug., 2012) 043504, [1111.2613].

[77] N. Mirabal, V. Frías-Martinez, T. Hassan and E. Frías-Martinez, Fermi's SIBYL: mining the gamma-ray sky for dark matter subhaloes, MNRAS 424 (July, 2012) L64-L68, [1205.4825].

[78] N. Mirabal, Dark matter versus pulsars: catching the impostor, MNRAS 436 (Dec., 2013) 2461-2464, [1309.3428].

[79] A. Berlin and D. Hooper, Stringent constraints on the dark matter annihilation cross section from subhalo searches with the Fermi Gamma-Ray Space Telescope, Phys. Rev. D 89 (Jan., 2014) 016014, [1309.0525].

[80] B. Bertoni, D. Hooper and T. Linden, Examining The Fermi-LAT Third Source Catalog in search of dark matter subhalos, J. Cosmology Astropart. Phys. 12 (Dec., 2015) 035, [1504.02087].

[81] D. Schoonenberg, J. Gaskins, G. Bertone and J. Diemand, Dark matter subhalos and unidentified sources in the Fermi 3FGL source catalog, J. Cosmology Astropart. Phys. 5 (May, 2016) 028, [1601.06781].

[82] B. S. Acharya, M. Actis, T. Aghajani, G. Agnetta, J. Aguilar, F. Aharonian et al., Introducing the CTA concept, Astropart. Phys. 43 (Mar., 2013) 3-18.

[83] K. Bernlöhr, A. Barnacka, Y. Becherini, O. Blanch Bigas, E. Carmona, P. Colin et al., Monte Carlo design studies for the Cherenkov Telescope Array, Astropart. Phys. 43 (Mar., 2013) 171-188, [1210.3503].

[84] P. Brun, E. Moulin, J. Diemand and J.-F. Glicenstein, Searches for dark matter subhaloes with wide-field Cherenkov telescope surveys, Phys. Rev. D 83 (Jan., 2011) 015003, [1012.4766].

[85] G. Dubus, J. L. Contreras, S. Funk, Y. Gallant, T. Hassan, J. Hinton et al., Surveys with the Cherenkov Telescope Array, Astropart. Phys. 43 (Mar., 2013) 317-330, [1208.5686].

[86] A. Charbonnier, C. Combet and D. Maurin, CLUMPY: A code for $\gamma$-ray signals from dark matter structures, Computer Physics Communications 183 (Mar., 2012) 656-668, [1201.4728].

[87] V. Bonnivard, M. Hütten, E. Nezri, A. Charbonnier, C. Combet and D. Maurin, CLUMPY: Jeans analysis, $\gamma$-ray and $\nu$ fluxes from dark matter (sub-)structures, Computer Physics Communications 200 (Mar., 2016) 336-349, [1506.07628].

[88] M. Doro, J. Conrad, D. Emmanoulopoulos, M. A. Sànchez-Conde, J. A. Barrio, E. Birsin et al., Dark matter and fundamental physics with the Cherenkov Telescope Array, Astropart. Phys. 43 (Mar., 2013) 189-214, [1208.5356].

[89] M. Wood, J. Buckley, S. Digel, S. Funk, D. Nieto and M. A. Sánchez-Conde, Prospects for Indirect Detection of Dark Matter with CTA, ArXiv e-prints (May, 2013) , [1305.0302].

[90] J. Carr, C. Balazs, T. Bringmann, T. Buanes, M. K. Daniel, M. Doro et al., Prospects for Indirect Dark Matter Searches with the Cherenkov Telescope Array (CTA), ArXiv e-prints (Aug., 2015), [1508.06128]. 
[91] H. Silverwood, C. Weniger, P. Scott and G. Bertone, A realistic assessment of the CTA sensitivity to dark matter annihilation, J. Cosmology Astropart. Phys. 3 (Mar., 2015) 055, [1408.4131].

[92] V. Lefranc, E. Moulin, P. Panci and J. Silk, Prospects for annihilating dark matter in the inner galactic halo by the Cherenkov Telescope Array, Phys. Rev. D 91 (June, 2015) 122003, [1502.05064].

[93] M. G. Walker, C. Combet, J. A. Hinton, D. Maurin and M. I. Wilkinson, Dark Matter in the Classical Dwarf Spheroidal Galaxies: A Robust Constraint on the Astrophysical Factor for

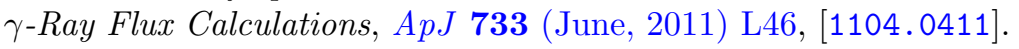

[94] A. Charbonnier, C. Combet, M. Daniel, S. Funk, J. A. Hinton, D. Maurin et al., Dark matter profiles and annihilation in dwarf spheroidal galaxies: prospectives for present and future $\gamma$-ray observatories - I. The classical dwarf spheroidal galaxies, MNRAS 418 (Dec., 2011) 1526-1556, [1104.0412].

[95] V. Bonnivard, C. Combet, D. Maurin and M. G. Walker, Spherical Jeans analysis for dark matter indirect detection in dwarf spheroidal galaxies - impact of physical parameters and triaxiality, MNRAS 446 (Jan., 2015) 3002-3021, [1407.7822].

[96] V. Bonnivard, C. Combet, M. Daniel, S. Funk, A. Geringer-Sameth, J. A. Hinton et al., Dark matter annihilation and decay in dwarf spheroidal galaxies: the classical and ultrafaint dSphs, MNRAS 453 (Oct., 2015) 849-867, [1504.02048].

[97] V. Bonnivard, C. Combet, D. Maurin, A. Geringer-Sameth, S. M. Koushiappas, M. G. Walker et al., Dark Matter Annihilation and Decay Profiles for the Reticulum II Dwarf Spheroidal Galaxy, ApJ 808 (Aug., 2015) L36, [1504.03309].

[98] V. Bonnivard, D. Maurin and M. G. Walker, Contamination of stellar-kinematic samples and uncertainty about dark matter annihilation profiles in ultrafaint dwarf galaxies: the example of Segue I, MNRAS 462 (Oct., 2016) 223-234, [1506.08209].

[99] M. G. Walker, M. Mateo, E. W. Olszewski, S. Koposov, V. Belokurov, P. Jethwa et al., Magellan/M2FS Spectroscopy of Tucana 2 and Grus 1, ApJ 819 (Mar., 2016) 53, [1511.06296].

[100] C. Combet, D. Maurin, E. Nezri, E. Pointecouteau, J. A. Hinton and R. White, Decaying dark matter: Stacking analysis of galaxy clusters to improve on current limits, Phys. Rev. D $\mathbf{8 5}$ (Mar., 2012) 063517, [1203.1164].

[101] D. Maurin, C. Combet, E. Nezri and E. Pointecouteau, Disentangling cosmic-ray and dark-matter induced $\gamma$-rays in galaxy clusters, A $\mathscr{J} A 547$ (Nov., 2012) A16, [1203.1166].

[102] M. Cirelli, G. Corcella, A. Hektor, G. Hütsi, M. Kadastik, P. Panci et al., PPPC \& DM ID: a poor particle physicist cookbook for dark matter indirect detection, J. Cosmology Astropart. Phys. 3 (Mar., 2011) 051, [1012.4515].

[103] L. Gao, J. F. Navarro, C. S. Frenk, A. Jenkins, V. Springel and S. D. M. White, The Phoenix Project: the dark side of rich Galaxy clusters, MNRAS 425 (Sept., 2012) 2169-2186, [1201.1940].

[104] P. Madau, J. Diemand and M. Kuhlen, Dark Matter Subhalos and the Dwarf Satellites of the Milky Way, ApJ 679 (June, 2008) 1260-1271, [0802.2265].

[105] P. Mollitor, E. Nezri and R. Teyssier, Baryonic and dark matter distribution in cosmological simulations of spiral galaxies, MNRAS 447 (Feb., 2015) 1353-1369, [1405.4318].

[106] T. Sawala, C. S. Frenk, A. Fattahi, J. F. Navarro, R. G. Bower, R. A. Crain et al., The APOSTLE simulations: solutions to the Local Group's cosmic puzzles, MNRAS 457 (Apr., 2016) 1931-1943, [1511.01098]. 
[107] A. R. Wetzel, P. F. Hopkins, J.-h. Kim, C.-A. Faucher-Giguère, D. Kereš and E. Quataert, Reconciling Dwarf Galaxies with $\Lambda$ CDM Cosmology: Simulating a Realistic Population of Satellites around a Milky Way-mass Galaxy, ApJ 827 (Aug., 2016) L23, [1602.05957].

[108] J. F. Navarro, A. Ludlow, V. Springel, J. Wang, M. Vogelsberger, S. D. M. White et al., The diversity and similarity of simulated cold dark matter haloes, MNRAS 402 (Feb., 2010) 21-34, [0810.1522].

[109] J. I. Read, The local dark matter density, Journal of Physics G Nuclear Physics 41 (June, 2014) 063101, [1404.1938].

[110] S. Garbari, C. Liu, J. I. Read and G. Lake, A new determination of the local dark matter density from the kinematics of K dwarfs, MNRAS 425 (Sept., 2012) 1445-1458, [1206.0015].

[111] B. Famaey, Dark Matter in the Milky Way, ArXiv e-prints (Jan., 2015) , [1501.01788].

[112] Z. M. Malkin, Analysis of determinations of the distance between the sun and the galactic center, Astronomy Reports 57 (Feb., 2013) 128-133, [1301.7011].

[113] F. V. Hessman, The difficulty of measuring the local dark matter density, A\&A $\mathbf{5 7 9}$ (July, 2015) A123, [1506.00384].

[114] F. Iocco, M. Pato and G. Bertone, Evidence for dark matter in the inner Milky Way, Nature Physics 11 (Mar., 2015) 245-248, [1502.03821].

[115] F. Nesti and P. Salucci, The Dark Matter halo of the Milky Way, AD 2013, J. Cosmology Astropart. Phys. 7 (July, 2013) 016, [1304.5127].

[116] J. Han, S. Cole, C. S. Frenk and Y. Jing, A unified model for the spatial and mass distribution of subhaloes, MNRAS 457 (Apr., 2016) 1208-1223, [1509.02175].

[117] K. J. Mack, Known unknowns of dark matter annihilation over cosmic time, MNRAS 439 (Apr., 2014) 2728-2735, [1309.7783].

[118] A. M. Green, S. Hofmann and D. J. Schwarz, The power spectrum of SUSY-CDM on subgalactic scales, MNRAS 353 (Sept., 2004) L23-L27, [astro-ph/0309621].

[119] R. E. Angulo, C. G. Lacey, C. M. Baugh and C. S. Frenk, The fate of substructures in cold dark matter haloes, MNRAS 399 (Oct., 2009) 983-995, [0810.2177].

[120] J. F. Navarro, C. S. Frenk and S. D. M. White, The Structure of Cold Dark Matter Halos, ApJ 462 (May, 1996) 563, [astro-ph/9508025].

[121] J. Einasto and U. Haud, Galactic models with massive corona. I - Method. II - Galaxy, AछA 223 (Oct., 1989) 89-106.

[122] T. Ishiyama, J. Makino and T. Ebisuzaki, Gamma-ray Signal from Earth-mass Dark Matter Microhalos, ApJ 723 (Nov., 2010) L195-L200, [1006.3392].

[123] D. Anderhalden and J. Diemand, Density profiles of CDM microhalos and their implications for annihilation boost factors, J. Cosmology Astropart. Phys. 4 (Apr., 2013) 009, [1302.0003].

[124] T. Ishiyama, Hierarchical Formation of Dark Matter Halos and the Free Streaming Scale, ApJ 788 (June, 2014) 27, [1404.1650].

[125] R. E. Angulo, O. Hahn, A. Ludlow and S. Bonoli, Earth-mass haloes and the emergence of NFW density profiles, ArXiv e-prints (Apr., 2016) , [1604.03131].

[126] M. A. Sánchez-Conde and F. Prada, The flattening of the concentration-mass relation towards low halo masses and its implications for the annihilation signal boost, MNRAS 442 (Aug., 2014) 2271-2277, [1312.1729].

[127] R. Bartels and S. Ando, Boosting the annihilation boost: Tidal effects on dark matter subhalos and consistent luminosity modeling, Phys. Rev. D 92 (Dec., 2015) 123508, [1507.08656]. 
[128] J. Zavala and N. Afshordi, Universal clustering of dark matter in phase space, MNRAS 457 (Mar., 2016) 986-992, [1508.02713].

[129] Á. Moliné, M. A. Sánchez-Conde, S. Palomares-Ruiz and F. Prada, Characterization of subhalo structural properties and implications for dark matter annihilation signals, ArXiv e-prints (Mar., 2016), [1603.04057].

[130] R. H. Wechsler, J. S. Bullock, J. R. Primack, A. V. Kravtsov and A. Dekel, Concentrations of Dark Halos from Their Assembly Histories, ApJ 568 (Mar., 2002) 52-70, [arXiv: astro-ph/0108151].

[131] J. S. Bullock, T. S. Kolatt, Y. Sigad, R. S. Somerville, A. V. Kravtsov, A. A. Klypin et al., Profiles of dark haloes: evolution, scatter and environment, MNRAS 321 (Mar., 2001) 559-575, [astro-ph/9908159].

[132] J. S. Bullock, Notes on the Missing Satellites Problem, ArXiv e-prints (Sept., 2010), [1009.4505].

[133] M. Boylan-Kolchin, J. S. Bullock and M. Kaplinghat, Too big to fail? The puzzling darkness of massive Milky Way subhaloes, MNRAS 415 (July, 2011) L40-L44, [1103.0007].

[134] A. Del Popolo, J. A. S. Lima, J. C. Fabris and D. C. Rodrigues, A unified solution to the small scale problems of the $\Lambda$ CDM model, J. Cosmology Astropart. Phys. 4 (Apr., 2014) 021, [1404.3674].

[135] A. J. Maxwell, J. Wadsley and H. M. P. Couchman, The Energetics of Cusp Destruction, ApJ 806 (June, 2015) 229, [1505.00825].

[136] M. Kuhlen, J. Diemand and P. Madau, The Shapes, Orientation, and Alignment of Galactic Dark Matter Subhalos, ApJ 671 (Dec., 2007) 1135-1146, [0705.2037].

[137] J. M. Siegal-Gaskins, Revealing dark matter substructure with anisotropies in the diffuse gamma-ray background, J. Cosmology Astropart. Phys. 10 (Oct., 2008) 040, [0807.1328].

[138] L. Gao, S. D. M. White, A. Jenkins, F. Stoehr and V. Springel, The subhalo populations of $\Lambda C D M$ dark haloes, MNRAS 355 (Dec., 2004) 819-834, [astro-ph/0404589].

[139] V. Springel, S. D. M. White, C. S. Frenk, J. F. Navarro, A. Jenkins, M. Vogelsberger et al., Prospects for detecting supersymmetric dark matter in the Galactic halo, Nature 456 (Nov., 2008) 73-76, [0809.0894].

[140] W. Atwood, A. Albert, L. Baldini, M. Tinivella, J. Bregeon, M. Pesce-Rollins et al., Pass 8: Toward the Full Realization of the Fermi-LAT Scientific Potential, ArXiv e-prints (Mar., 2013), [1303.3514].

[141] K. Hayashi, K. Ichikawa, S. Matsumoto, M. Ibe, M. N. Ishigaki and H. Sugai, Dark matter annihilation and decay from non-spherical dark halos in galactic dwarf satellites, MNRAS 461 (Sept., 2016) 2914-2928, [1603.08046].

[142] K. M. Górski, E. Hivon, A. J. Banday, B. D. Wandelt, F. K. Hansen, M. Reinecke et al., HEALPix: A Framework for High-Resolution Discretization and Fast Analysis of Data Distributed on the Sphere, ApJ 622 (Apr., 2005) 759-771, [astro-ph/0409513].

[143] M. Ackermann, M. Ajello, A. Albert, L. Baldini, J. Ballet, G. Barbiellini et al., Anisotropies in the diffuse gamma-ray background measured by the Fermi LAT, Phys. Rev. D 85 (Apr., 2012) 083007, [1202.2856].

[144] S. Ando, Gamma-ray background anisotropy from Galactic dark matter substructure, Phys. Rev. D 80 (July, 2009) 023520, [0903.4685].

[145] J. U. Lange and M.-C. Chu, Can galactic dark matter substructure contribute to the cosmic gamma-ray anisotropy?, MNRAS 447 (Feb., 2015) 939-947, [1412.5749]. 
[146] M. Fornasa, J. Zavala, M. A. Sánchez-Conde, J. M. Siegal-Gaskins, T. Delahaye, F. Prada et al., Characterization of dark-matter-induced anisotropies in the diffuse gamma-ray background, MNRAS 429 (Feb., 2013) 1529-1553, [1207.0502].

[147] F. Calore, V. De Romeri, M. Di Mauro, F. Donato, J. Herpich, A. V. Macciò et al., $\gamma$-ray anisotropies from dark matter in the Milky Way: the role of the radial distribution, MNRAS 442 (Aug., 2014) 1151-1156, [1402.0512].

[148] S. Ando and E. Komatsu, Constraints on the annihilation cross section of dark matter particles from anisotropies in the diffuse gamma-ray background measured with Fermi-LAT, Phys. Rev. D 87 (June, 2013) 123539, [1301.5901].

[149] A. Cuoco, E. Komatsu and J. M. Siegal-Gaskins, Joint anisotropy and source count constraints on the contribution of blazars to the diffuse gamma-ray background, Phys. Rev. D 86 (Sept., 2012) 063004, [1202.5309].

[150] M. Di Mauro, A. Cuoco, F. Donato and J. M. Siegal-Gaskins, Fermi-LAT $\gamma$-ray anisotropy and intensity explained by unresolved radio-loud active galactic nuclei, J. Cosmology Astropart. Phys. 11 (Nov., 2014) 021, [1407.3275].

[151] G. A. Gómez-Vargas, A. Cuoco, T. Linden, M. A. Sánchez-Conde, J. M. Siegal-Gaskins, T. Delahaye et al., Dark matter implications of Fermi-LAT measurement of anisotropies in the diffuse gamma-ray background, Nuclear Instruments and Methods in Physics Research A 742 (Apr., 2014) 149-153, [1303.2154].

[152] J. M. Siegal-Gaskins, Separating astrophysical sources from indirect dark matter signals, Proceedings of the National Academy of Science 112 (Oct., 2015) 12272-12277.

[153] J. Ripken, A. Cuoco, H.-S. Zechlin, J. Conrad and D. Horns, The sensitivity of Cherenkov telescopes to dark matter and astrophysical anisotropies in the diffuse gamma-ray background, J. Cosmology Astropart. Phys. 1 (Jan., 2014) 049, [1211.6922].

[154] N. Mirabal, E. Charles, E. C. Ferrara, P. L. Gonthier, A. K. Harding, M. A. Sánchez-Conde et al., 3FGL Demographics Outside the Galactic Plane using Supervised Machine Learning: Pulsar and Dark Matter Subhalo Interpretations, ApJ 825 (July, 2016) 69, [1605.00711].

[155] Kevin Meagher for the VERITAS Collaboration, Six years of VERITAS observations of the Crab Nebula, ArXiv e-prints (Aug., 2015) , [1508.06442].

[156] M. Ackermann, M. Ajello, A. Albert, W. B. Atwood, L. Baldini, J. Ballet et al., The Spectrum of Isotropic Diffuse Gamma-Ray Emission between $100 \mathrm{MeV}$ and 820 GeV, ApJ 799 (Jan., 2015) 86, [1410.3696].

[157] J. Knödlseder, M. Mayer, C. Deil, J.-B. Cayrou, E. Owen, N. Kelley-Hoskins et al., GammaLib and ctools: A software framework for the analysis of astronomical gamma-ray data, AESA (June, 2016) in press, [1606.00393].

[158] V. Lefranc, E. Moulin and for the H. E. S. S. collaboration, Dark matter search in the inner Galactic halo with H.E.S.S. I and H.E.S.S. II, ArXiv e-prints (Sept., 2015) , [1509.04123].

[159] P. J. E. Peebles, The large-scale structure of the universe. 1980.

[160] G. L. Bryan and M. L. Norman, Statistical Properties of X-Ray Clusters: Analytic and Numerical Comparisons, ApJ 495 (Mar., 1998) 80-99, [astro-ph/9710107].

[161] Planck Collaboration, P. A. R. Ade, N. Aghanim, C. Armitage-Caplan, M. Arnaud, M. Ashdown et al., Planck 2013 results. XVI. Cosmological parameters, A\&A 571 (Nov., 2014) A16, [1303.5076].

[162] M. White, The mass of a halo, A\&A 367 (Feb., 2001) 27-32, [astro-ph/0011495].

[163] B. Diemer and A. V. Kravtsov, A Universal Model for Halo Concentrations, ApJ 799 (Jan., 2015) 108, [1407.4730]. 
[164] C. Giocoli, G. Tormen, R. K. Sheth and F. C. van den Bosch, The substructure hierarchy in dark matter haloes, MNRAS 404 (May, 2010) 502-517, [0911.0436].

[165] S. Garrison-Kimmel, M. Boylan-Kolchin, J. S. Bullock and K. Lee, ELVIS: Exploring the Local Volume in Simulations, MNRAS 438 (Mar., 2014) 2578-2596, [1310.6746].

[166] E. Komatsu, K. M. Smith, J. Dunkley, C. L. Bennett, B. Gold, G. Hinshaw et al., Seven-year Wilkinson Microwave Anisotropy Probe (WMAP) Observations: Cosmological Interpretation, ApJS 192 (Feb., 2011) 18, [1001.4538].

[167] D. Coe, Dark Matter Halo Mass Profiles, ArXiv e-prints (May, 2010) , [1005.0411].

[168] P. Schechter, An analytic expression for the luminosity function for galaxies., ApJ 203 (Jan., 1976) 297-306.

[169] R. L. da Silva, M. R. Krumholz, M. Fumagalli and S. M. Fall, An analytic method to compute star cluster luminosity statistics, MNRAS 438 (Mar., 2014) 2355-2370.

[170] S. Ando and E. Komatsu, Anisotropy of the cosmic gamma-ray background from dark matter annihilation, Phys. Rev. D 73 (Jan., 2006) 023521, [astro-ph/0512217].

[171] N. F. Martin, M. Geha, R. A. Ibata, M. L. M. Collins, B. P. M. Laevens, E. F. Bell et al., Is Draco II one of the faintest dwarf galaxies? First study from Keck/DEIMOS spectroscopy, MNRAS 458 (May, 2016) L59-L63, [1510.01326].

[172] S. E. Koposov, A. R. Casey, V. Belokurov, J. R. Lewis, G. Gilmore, C. Worley et al., Kinematics and Chemistry of Recently Discovered Reticulum 2 and Horologium 1 Dwarf Galaxies, ApJ 811 (Sept., 2015) 62, [1504.07916].

[173] E. N. Kirby, J. D. Simon and J. G. Cohen, Spectroscopic Confirmation of the Dwarf Galaxies Hydra II and Pisces II and the Globular Cluster Laevens 1, ApJ 810 (Sept., 2015) 56, [1506.01021].

[174] E. N. Kirby, J. G. Cohen, J. D. Simon and P. Guhathakurta, Triangulum II: Possibly a Very Dense Ultra-faint Dwarf Galaxy, ApJ 814 (Nov., 2015) L7, [1510.03856].

[175] N. F. Martin, R. A. Ibata, M. L. M. Collins, R. M. Rich, E. F. Bell, A. M. N. Ferguson et al., Triangulum II: A Very Metal-poor and Dynamically Hot Stellar System, ApJ $\mathbf{8 1 8}$ (Feb., 2016) 40, [1510.04433].

[176] A. Koch, M. I. Wilkinson, J. T. Kleyna, M. Irwin, D. B. Zucker, V. Belokurov et al., A Spectroscopic Confirmation of the Bootes II Dwarf Spheroidal, ApJ 690 (Jan., 2009) 453-462, [0809.0700].

[177] A. Koch and R. M. Rich, A Chemical Confirmation of the Faint Boötes II Dwarf Spheroidal Galaxy, ApJ 794 (Oct., 2014) 89, [1408.3628].

[178] N. F. Martin, D. L. Nidever, G. Besla, K. Olsen, A. R. Walker, A. K. Vivas et al., Hydra II: A Faint and Compact Milky Way Dwarf Galaxy Found in the Survey of the Magellanic Stellar History, ApJ 804 (May, 2015) L5, [1503.06216].

[179] B. Willman, M. Geha, J. Strader, L. E. Strigari, J. D. Simon, E. Kirby et al., Willman 1-A Probable Dwarf Galaxy with an Irregular Kinematic Distribution, AJ 142 (Oct., 2011) 128, [1007.3499].

[180] S. E. Koposov, V. Belokurov, D. B. Zucker, G. F. Lewis, R. A. Ibata, E. W. Olszewski et al., Exposing Sgr tidal debris behind the Galactic disc with M giants selected in WISEก2MASS, MNRAS 446 (Jan., 2015) 3110-3117, [1410.6482].

[181] J. Binney and S. Tremaine, Galactic Dynamics: Second Edition. Princeton University Press, 2008.

[182] A. Genina and M. Fairbairn, The Potential of the Dwarf Galaxy Triangulum II for Dark Matter Indirect Detection, ArXiv e-prints (Apr., 2016) , [1604.00838]. 
[183] K. Bekki and S. Stanimirović, The total mass and dark halo properties of the Small Magellanic Cloud, MNRAS 395 (May, 2009) 342-350, [0807.2102].

[184] G. Pietrzyński, D. Graczyk, W. Gieren, I. B. Thompson, B. Pilecki, A. Udalski et al., An eclipsing-binary distance to the Large Magellanic Cloud accurate to two per cent, Nature 495 (Mar., 2013) 76-79, [1303.2063].

[185] G. Besla, The Orbits and Total Mass of the Magellanic Clouds, ArXiv e-prints (Nov., 2015), [1511.03346].

[186] D. Graczyk, G. Pietrzyński, I. B. Thompson, W. Gieren, B. Pilecki, P. Konorski et al., The Araucaria Project. The Distance to the Small Magellanic Cloud from Late-type Eclipsing Binaries, ApJ 780 (Jan., 2014) 59, [1311.2340].

[187] R. Caputo, M. R. Buckley, P. Martin, E. Charles, A. M. Brooks, A. Drlica-Wagner et al., Search for gamma-ray emission from dark matter annihilation in the Small Magellanic Cloud with the Fermi Large Area Telescope, Phys. Rev. D 93 (Mar., 2016) 062004, [1603.00965].

[188] G. Cowan, K. Cranmer, E. Gross and O. Vitells, Asymptotic formulae for likelihood-based tests of new physics, European Physical Journal C 71 (Feb., 2011) 1554, [1007.1727].

[189] J. Conrad, Statistical issues in astrophysical searches for particle dark matter, Astropart. Phys. 62 (Mar., 2015) 165-177, [1407.6617]. 